\title{
Gold(I)-Catalyzed Enantioselective Cyclopropanation of $\alpha$-Aryl Diazoacetates with Enamides
}

Zhaowei Chu, Zhiqiong Tang, Kenan Zhang, Lei Wang, Wenbo Li*, Hai-Hong Wu* and Junliang

$$
\text { Zhang* }
$$

Shanghai Key Laboratory of Green Chemistry and Chemical Processes, School of Chemistry and Molecular Engineering, East China Normal University, 3663 North Zhongshan Road, Shanghai 200062, P. R. China.

Fax:(+86)-021-6223-3213; E-mail : junliangzhang@fudan.edu.cn

\section{Table of Content}

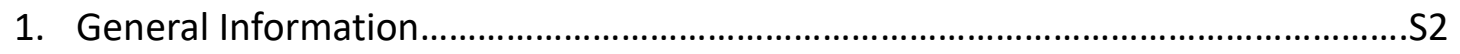

2. General procedure for synthesis of racemic compounds .....................................S2

3. General procedure for asymmetric synthesis of compounds

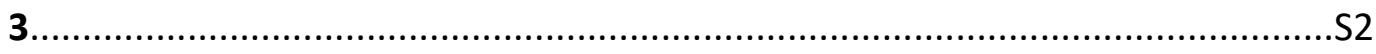

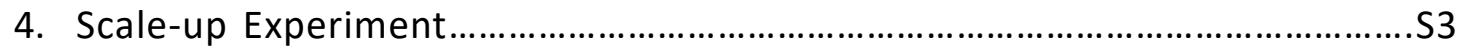

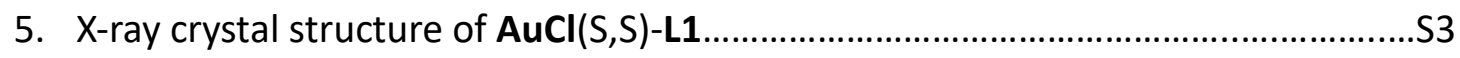

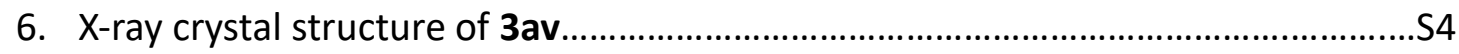

7. ${ }^{1} \mathrm{H},{ }^{13} \mathrm{C}$ NMR, and HPLC spectra for compounds 3ae-3av, and 3be-3bv..................S5 


\section{General Information}

Unless otherwise noted, all reactions were carried out in standard Schlenk techniques with magnetic stirring bar under argon atmosphere. Materials obtained from commercial suppliers were used directly without further purification. ${ }^{1} \mathrm{H}$ NMR spectra were recorded on a BRUKER 500 (500 $\mathrm{MHz}$ ), BRUKER 400 (400 MHz) spectrometer in $\mathrm{CDCl}_{3}$. Chemical shifts are reported in ppm with tetramethylsilane (TMS: $0 \mathrm{ppm}$ ) with the solvent resonance as the internal standard. Data are reported as follows: chemical shift, multiplicity $(\mathrm{s}=$ singlet, $\mathrm{d}=$ doublet, $\mathrm{t}=$ triplet, $\mathrm{q}=$ quartet, $\mathrm{m}$ $=$ multiplet), coupling constants $(\mathrm{Hz})$, and integration. ${ }^{13} \mathrm{C}$ NMR spectra were recorded on a BRUKER 500 (125 MHz), BRUKER 400 (100 MHz) spectrometer in $\mathrm{CDCl}_{3}$ with complete proton decoupling. Chemical shifts are reported in ppm with the deuterium solvent as the internal standard (e.g. $\mathrm{CDCl}_{3}: 77.0 \mathrm{ppm}$ ). The $[\alpha]^{\mathrm{D}}$ was recorded using PolAAr 3005 High Accuracy Polarimeter. Infrared (IR) spectra were obtained using a Bruker tensor 27 infrared spectrometer. The ee was recorded using UltiMate 3000 HPLC from Dionex Company.

Anhydrous tetrahydrofuran (THF) was dried with sodium benzophenone and distilled before use; anhydrous dichloromethane (DCM), was purified by distillation over $\mathrm{CaH} 2$ prior to use; $\mathrm{AgOTf}$,

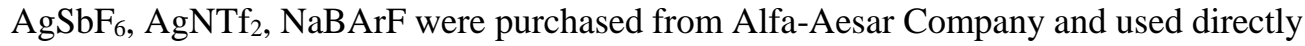

Reactions were monitored by thin layer chromatography (TLC) using silicycle pre-coated silica gel plates. Flash column chromatography was performed on silica gel 60 (particle size 200-400 mesh ASTM, purchased from Yantai, China) and eluted with hexane/ethyl acetate or hexane/ $\mathrm{CH}_{2} \mathrm{Cl}_{2}$.

\section{General procedures for synthesis of racemic compounds 3}

In a flame dried $10-\mathrm{mL}$ Schlenk tube equipped with a magnetic stir bar, enamide $(0.1 \mathrm{mmol})$ and $\mathrm{AgSbF}_{6}(5.0 \mathrm{~mol} \%)$ were dissolved in dry DCM $(1 \mathrm{~mL})$. A solution of the diazo ester $(0.2 \mathrm{mmol}$, 2.0 equiv) in dry DCM (1 mL) was added using a syringe for the duration of $15 \mathrm{~min}$. Afterward, the reaction mixture was stirred for further 5 min and concentrated under reduced pressure. The residue was purified by column chromatography on silica gel using hexanes and ethyl acetate as eluents

\section{General procedures for asymmetric synthesis of compounds 3}

In a flame dried $10-\mathrm{mL}$ Schlenk tube, after the solution of ligand $\mathbf{L 1}(5.5 \mathrm{~mol} \%)$ and $\mathrm{Me}_{2} \mathrm{SAuCl}$ $(5.0 \mathrm{~mol} \%)$ in anhydrous DCM $(1 \mathrm{~mL})$ was stirred at room temperature for $2 \mathrm{~h}$, the solvent was removed in vacuum. A solution of $\mathrm{AgSbF}_{6}(5.0 \mathrm{~mol} \%)$ in anhydrous $\mathrm{DCM}(1 \mathrm{~mL})$ was added to the residue and the mixture was stirred at $\mathrm{rt}$ for $15 \mathrm{mins}$. Then the enamide $(0.1 \mathrm{mmol})$ were added to 
the mixture. A solution of the diazo ester $(0.2 \mathrm{mmol}, 2.0$ equiv) in dry DCM $(1 \mathrm{~mL})$ was added using a syringe for the duration of $15 \mathrm{~min}$. Afterward, the reaction mixture was stirred for further 5 min and concentrated under reduced pressure. The residue was purified by column chromatography on silica gel using hexanes and ethyl acetate as eluents.

\section{Scale-up Experiment}

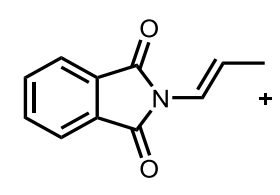

1a (748 mg, $4 \mathrm{mmol})$<smiles>CCCc1cccc(P)c1OC(=N)C(=N)c1ccccc1</smiles>

$2 \mathrm{e}(1.9 \mathrm{~g}, 6 \mathrm{mmol})$

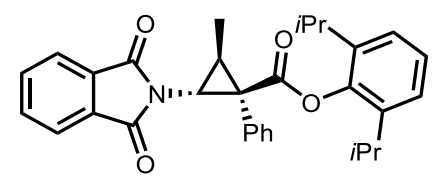

3ae

$1.03 \mathrm{~g}, 52 \%$ yield, $95 \%$ ee

In a flame dried 100-mL Schlenk flask, after the solution of ligand L1 (2.75 mol\%) and $\mathrm{Me}_{2} \mathrm{SAuCl}(2.5 \mathrm{~mol} \%)$ in anhydrous DCM $(20 \mathrm{~mL})$ was stirred at room temperature for $2 \mathrm{~h}$, the solvent was removed in vacuum. A solution of AgSbF6 (2.5 mol\%) in anhydrous DCM (20 mL) was added to the residue and the mixture was stirred at $\mathrm{rt}$ for $15 \mathrm{mins}$. Then the enamide $(4.0 \mathrm{mmol})$ were added to the mixture. A solution of the diazo ester ( $6.0 \mathrm{mmol}, 1.5$ equiv) in dry DCM (20 mL) was added using a syringe for the duration of $30 \mathrm{~min}$. Afterward, the reaction mixture was stirred for further $5 \mathrm{~min}$ and concentrated under reduced pressure. The residue was purified by column chromatography on silica gel using hexanes and ethyl acetate as eluents.

\section{X-ray crystal structure of AuCl(S,S)-L1}

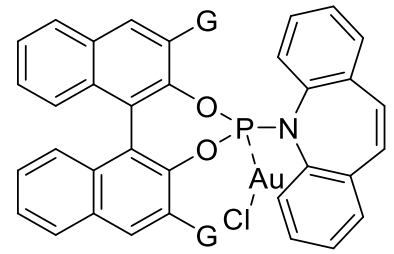

$\mathrm{AuCl}(\mathrm{S}, \mathrm{S})-\mathrm{L} 1, \mathrm{G}=3,5-\left(\mathrm{CF}_{3}\right)_{2} \mathrm{C}_{6} \mathrm{H}_{3}$
L1AuCl (2.75 mol\%) $\underset{\mathrm{DCM}, \mathrm{rt}, 20 \mathrm{~min}}{\stackrel{\mathrm{AgSbF}_{6}(2.5 \mathrm{mo} \%)}{\longrightarrow}}$ 然 
c/Å

$\alpha /{ }^{\circ}$

$\beta /{ }^{\circ}$

$\gamma /{ }^{\circ}$

Volume $/ \AA^{3}$

$\mathrm{Z}$

$\rho_{\text {calc }} \mathrm{g} / \mathrm{cm}^{3}$

$\mu / \mathrm{mm}^{-1}$

$\mathrm{F}(000)$

Crystal size $/ \mathrm{mm}^{3}$

Radiation

$2 \Theta$ range for data collection/ ${ }^{\circ}$

Index ranges

Reflections collected

Independent reflections

Data/restraints/parameters

Goodness-of-fit on $\mathrm{F}^{2}$

Final $\mathrm{R}$ indexes $[\mathrm{I}>=2 \sigma(\mathrm{I})]$

Final R indexes [all data]

Largest diff. peak/hole / e $\AA^{-3}$

Flack parameter
29.9435(2)

90

90

90

4867.54(7)

4

1.589

7.255

2272.0

$0.42 \times 0.08 \times 0.05$

$\mathrm{CuK} \alpha(\lambda=1.54184)$

7.436 to 149.114

$$
-10 \leq \mathrm{h} \leq 10,-24 \leq \mathrm{k} \leq 24,-37 \leq 1 \leq 37
$$

130298

$$
9860\left[R_{\text {int }}=0.1145, R_{\text {sigma }}=0.0409\right]
$$

9860/114/613

$$
1.023
$$

$\mathrm{R}_{1}=0.0505, \mathrm{wR}_{2}=0.1205$

$\mathrm{R}_{1}=0.0521, \mathrm{wR}_{2}=0.1216$

$1.28 /-1.16$

$0.006(5)$

\section{X-ray crystal structure of 3av}
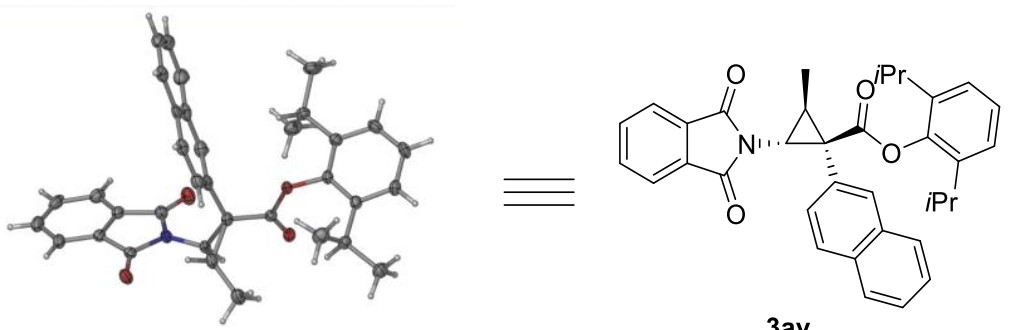

$3 \mathrm{av}$

Identification code

Empirical formula

Formula weight

Temperature/K

Crystal system

Space group

$$
\begin{aligned}
& \mathrm{a} / \AA \\
& \mathrm{b} / \AA \\
& \mathrm{c} / \AA \\
& \alpha /{ }^{\circ} \\
& \beta /{ }^{\circ}
\end{aligned}
$$

$\mathrm{C}_{35} \mathrm{H}_{33} \mathrm{NO}_{4}$

531.62

100.00(10)

orthorhombic

$\mathrm{P} 2{ }_{1} 2_{1} 2_{1}$

$8.72500(10)$

$15.9684(2)$

20.2607(2)

90

90 
$\gamma /{ }^{\circ}$

Volume $/ \AA^{3}$

Z

$\rho_{\text {calc }} / \mathrm{cm}^{3}$

$\mu / \mathrm{mm}^{-1}$

$\mathrm{F}(000)$

Crystal size $/ \mathrm{mm}^{3}$

Radiation
90

2822.81(6)

4

1.251

0.646

1128.0

$0.46 \times 0.38 \times 0.32$

$\operatorname{CuK} \alpha(\lambda=1.54184)$

8.728 to 134.156

$2 \Theta$ range for data collection $/^{\circ}$

Index ranges

$-10 \leqslant \mathrm{~h} \leqslant 10,-19 \leqslant \mathrm{k} \leqslant 18,-24 \leqslant 1 \leqslant 24$

Reflections collected

65523

Independent reflections

Data/restraints/parameters

Goodness-of-fit on $\mathrm{F}^{2}$

Final $R$ indexes $[\mathrm{I}>=2 \sigma(\mathrm{I})]$

Final R indexes [all data]

Largest diff. peak/hole / e $\AA^{-3}$

Flack parameter

$5028[$ Rint $=0.0853$, Rsigma $=0.0265]$

$5028 / 0 / 366$

1.067

$\mathrm{R} 1=0.0427, \mathrm{wR} 2=0.1137$

$\mathrm{R} 1=0.0434, \mathrm{wR} 2=0.1143$

$0.57 /-0.17$

$0.05(8)$

\section{7. ${ }^{1} \mathrm{H},{ }^{13} \mathrm{C}$ NMR, and HPLC spectra}
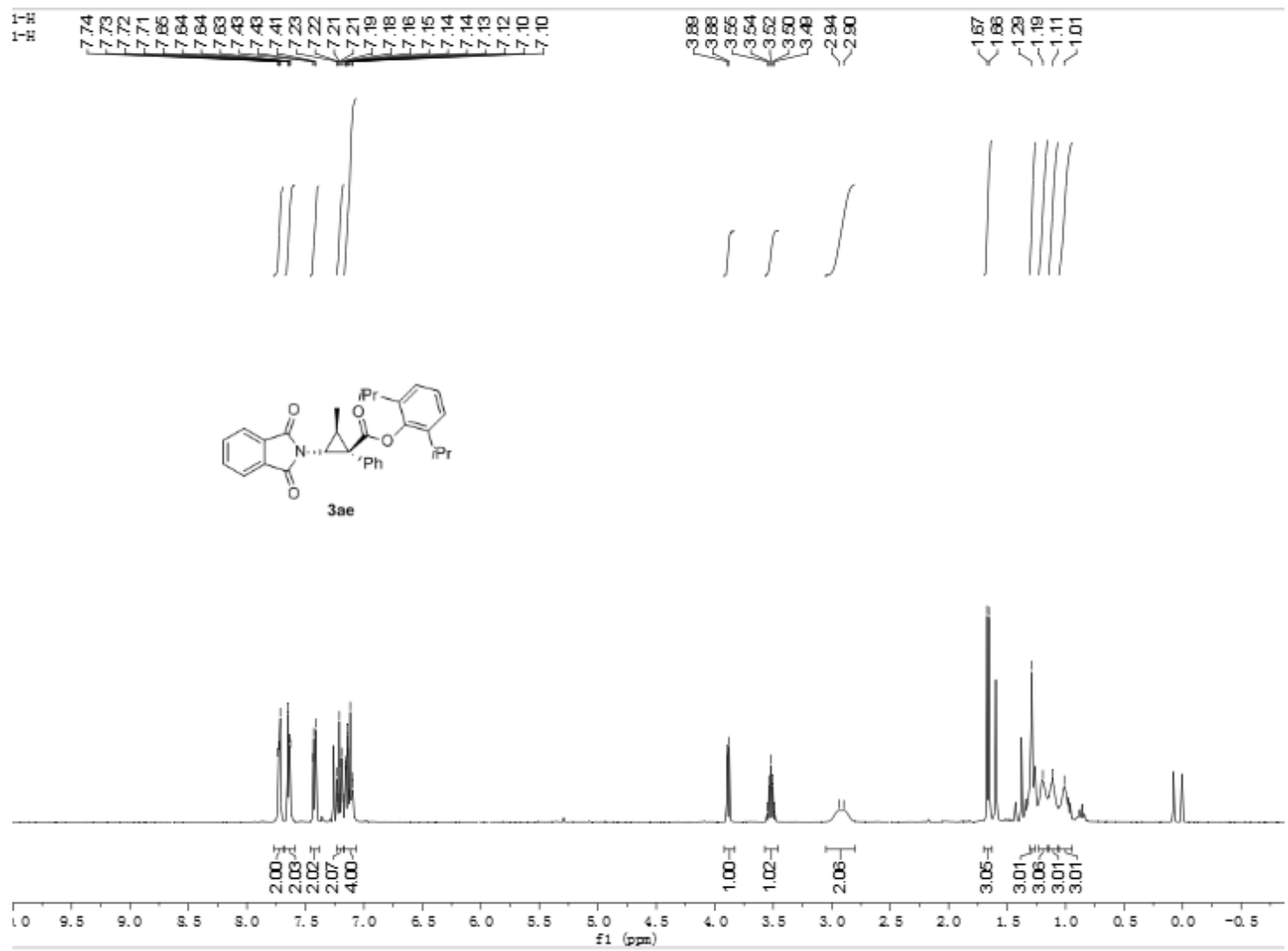

Figure S1: ${ }^{1} \mathrm{H}$ NMR (400 MHz, $\mathrm{CDCl}_{3}$ ) spectrum for 3ae 


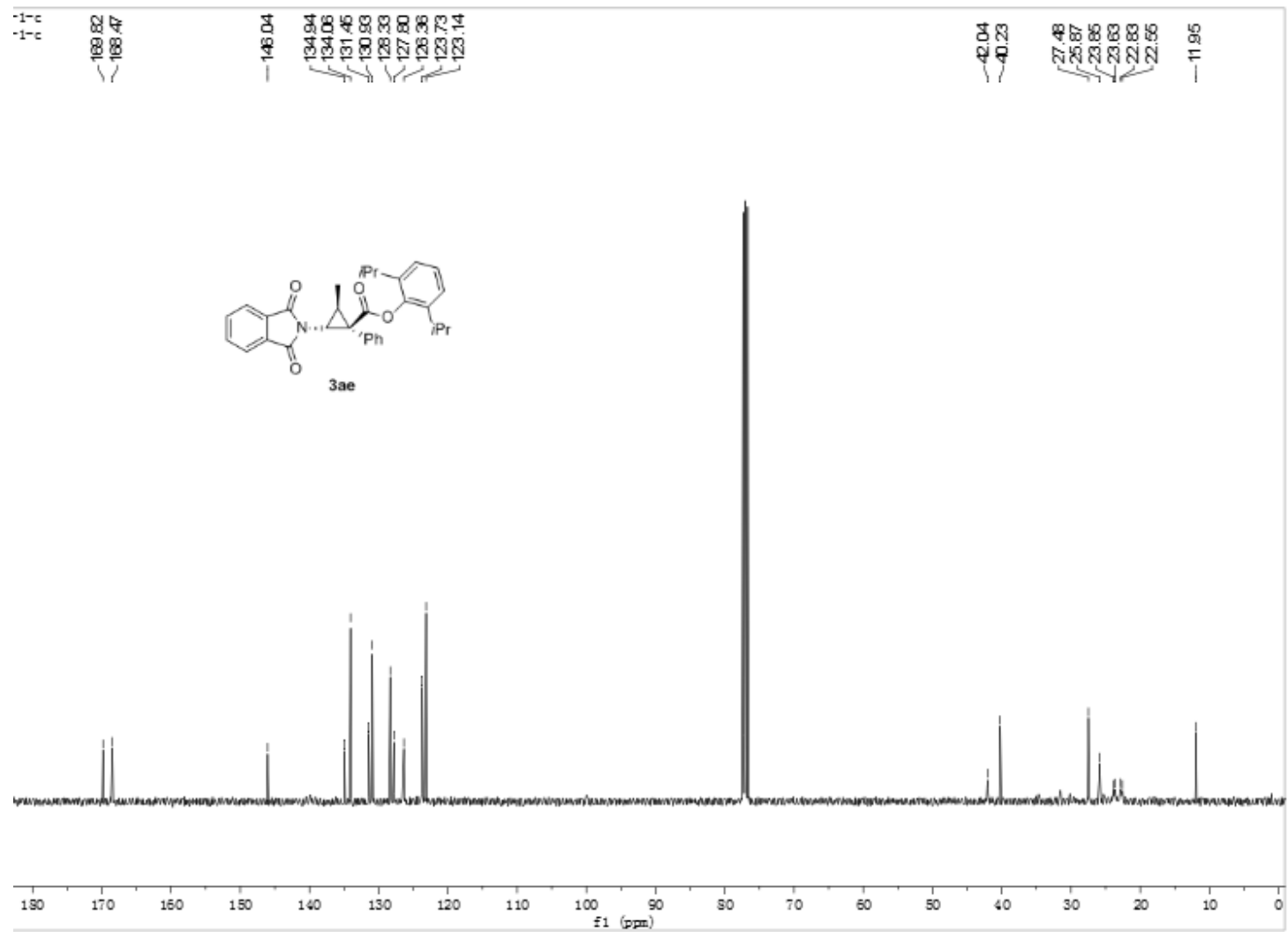

Figure S2: ${ }^{13} \mathrm{C} \mathrm{NMR}\left(100 \mathrm{MHz}, \mathrm{CDCl}_{3}\right)$ spectrum for $\mathbf{3 a e}$

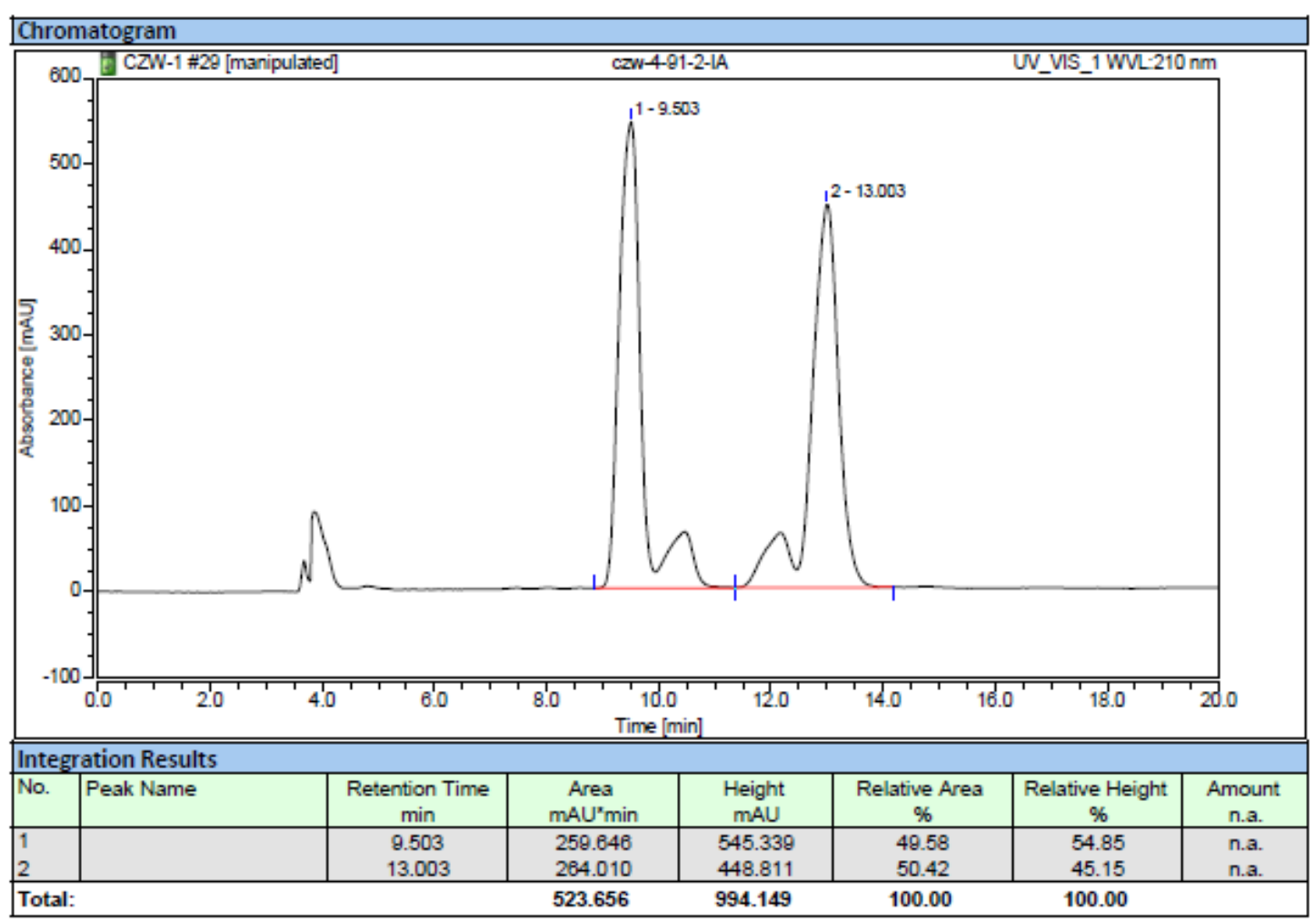




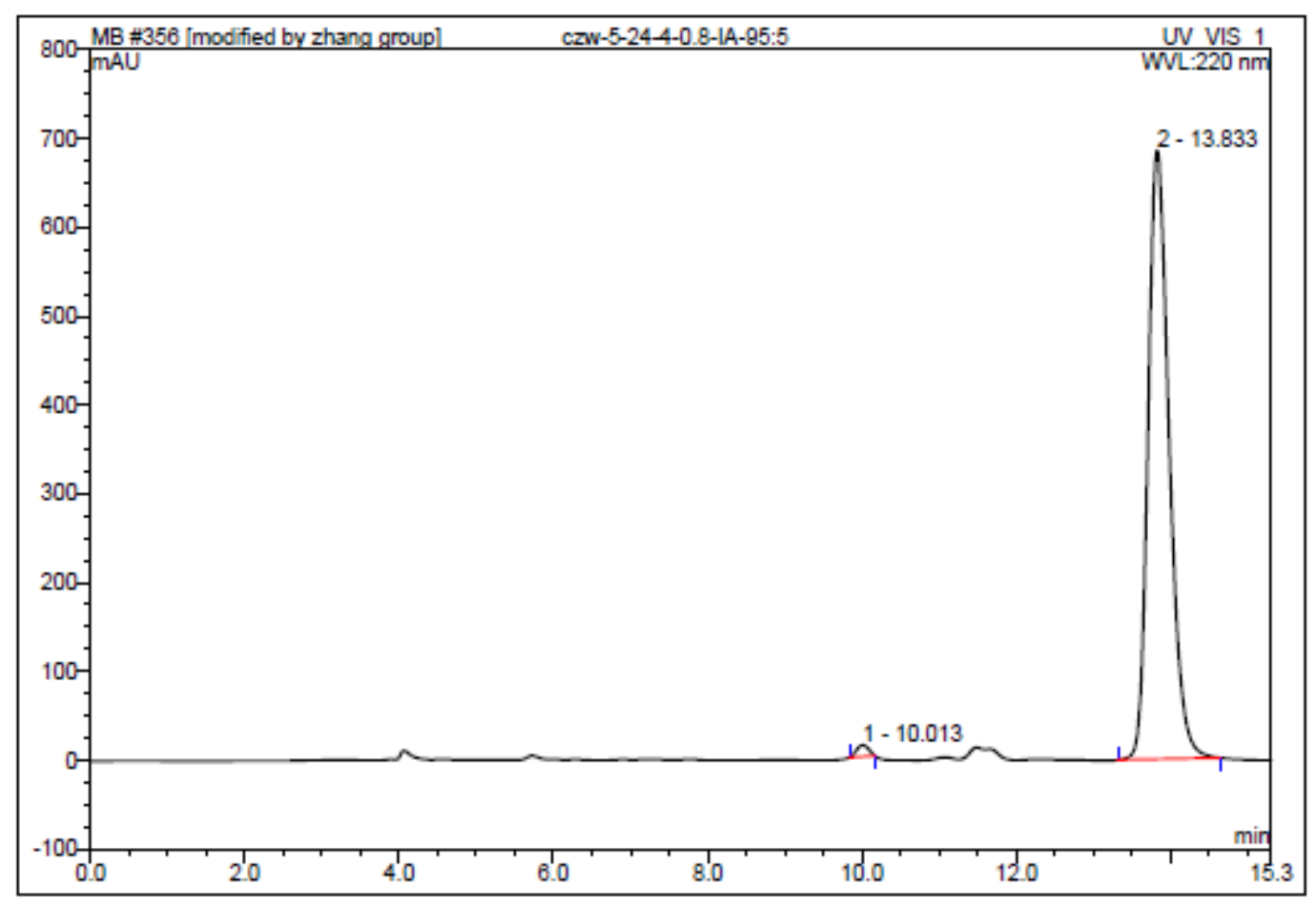

\begin{tabular}{|r|ccrrrrr|}
\hline No. & $\begin{array}{c}\text { Ret.Time } \\
\text { min }\end{array}$ & Peak Name & $\begin{array}{c}\text { Height } \\
\text { mAU }\end{array}$ & $\begin{array}{c}\text { Area } \\
\text { mAU*min }\end{array}$ & $\begin{array}{c}\text { Rel.Area } \\
\%\end{array}$ & Amount & Type \\
\hline 1 & 10.01 & n.a. & 12.953 & 2.323 & 1.06 & n.a. & BMB $^{\star}$ \\
2 & 13.83 & n.a. & 685.318 & 216.862 & 98.94 & n.a. & BMB $^{\star}$ \\
\hline Total: & & & 698.270 & 219.185 & 100.00 & 0.000 & \\
\hline
\end{tabular}

Figure S3: HPLC chromatographs of 3ae

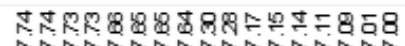

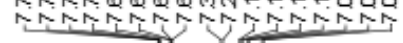

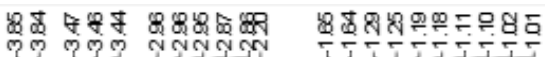

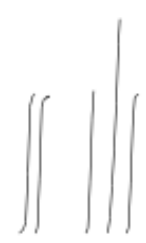

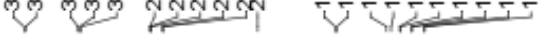

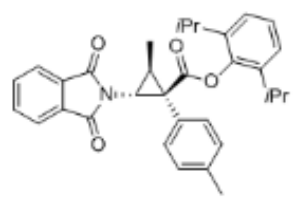

3af

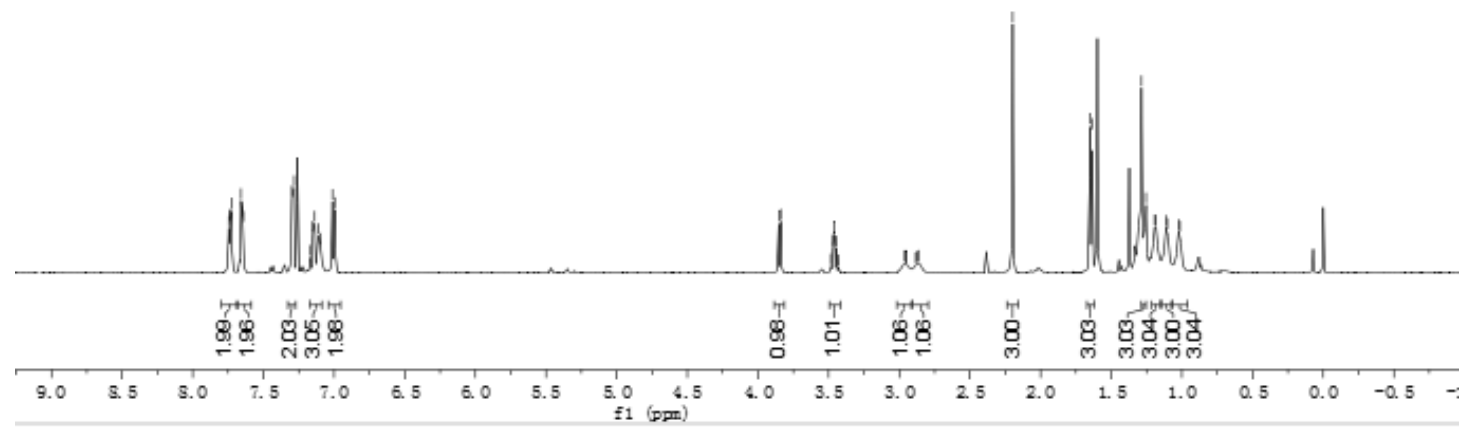


Figure S4: ${ }^{1} \mathrm{H}$ NMR $\left(500 \mathrm{MHz}, \mathrm{CDCl}_{3}\right)$ spectrum for 3af

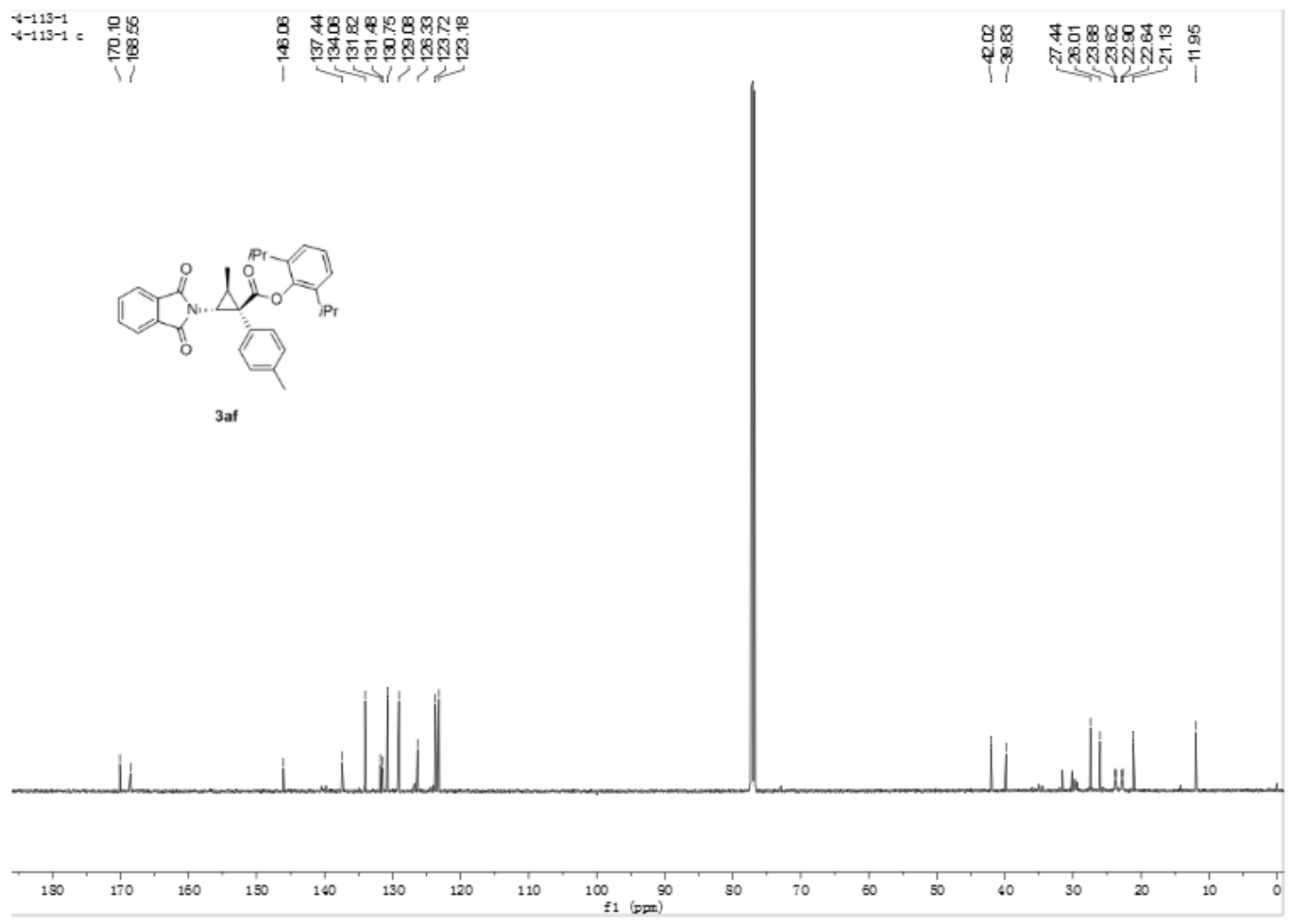

Figure S5: ${ }^{13} \mathrm{C}$ NMR $\left(125 \mathrm{MHz}, \mathrm{CDCl}_{3}\right)$ spectrum for $\mathbf{3 a f}$

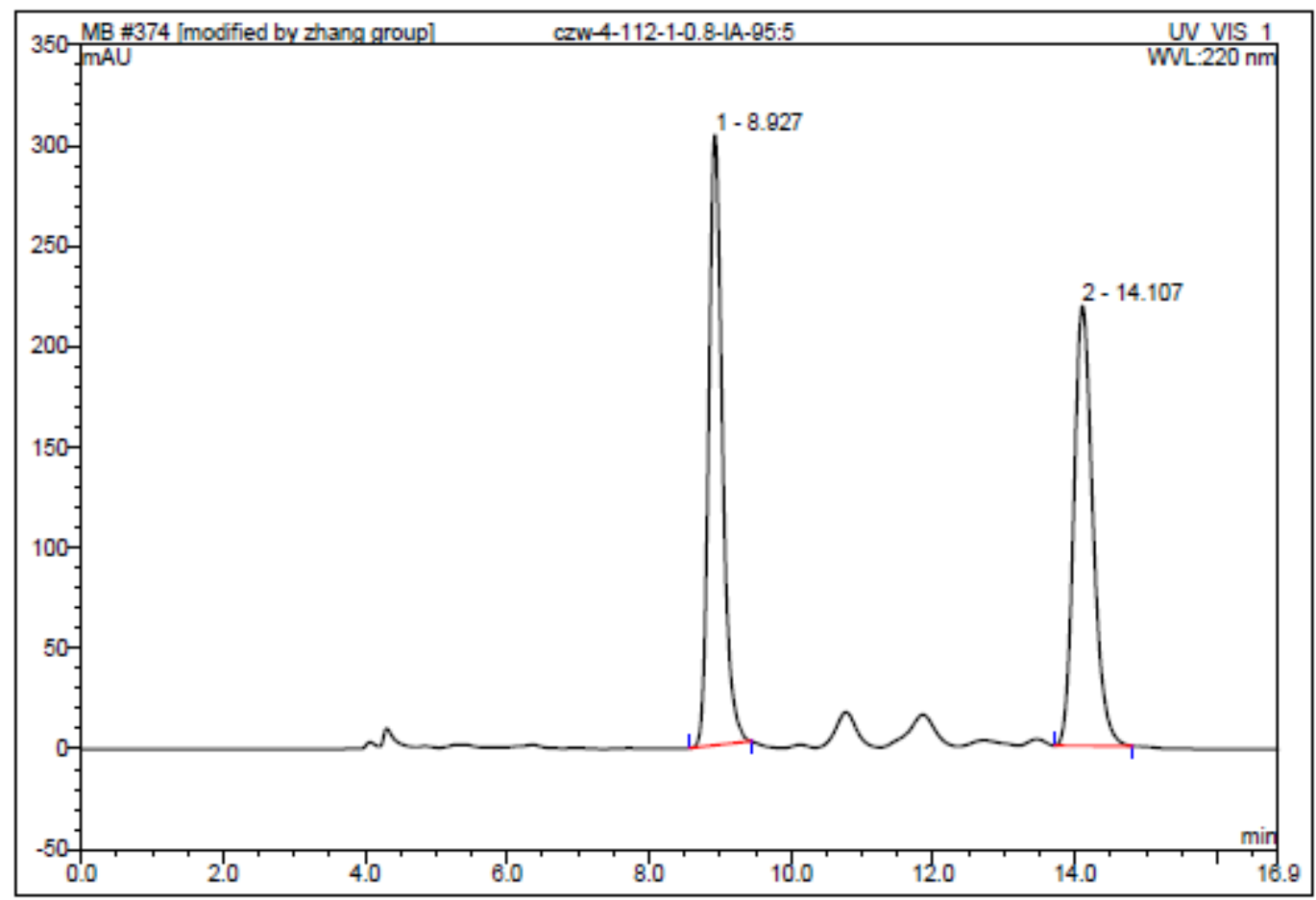

\begin{tabular}{|r|rrrrrrr|}
\hline No. & $\begin{array}{c}\text { Ret.Time } \\
\text { min }\end{array}$ & Peak Name & $\begin{array}{c}\text { Height } \\
\text { mAU }\end{array}$ & $\begin{array}{c}\text { Area } \\
\text { mAU*min }\end{array}$ & $\begin{array}{r}\text { Rel.Area } \\
\%\end{array}$ & Amount & Type \\
\hline 1 & 8.93 & n.a. & 303.569 & 67.902 & 49.96 & n.a. & BMB $^{\star}$ \\
2 & 14.11 & n.a. & 218.586 & 67.999 & 50.04 & n.a. & BMB $^{\star}$ \\
\hline Total: & & & 522.154 & 135.902 & 100.00 & 0.000 & \\
\hline
\end{tabular}




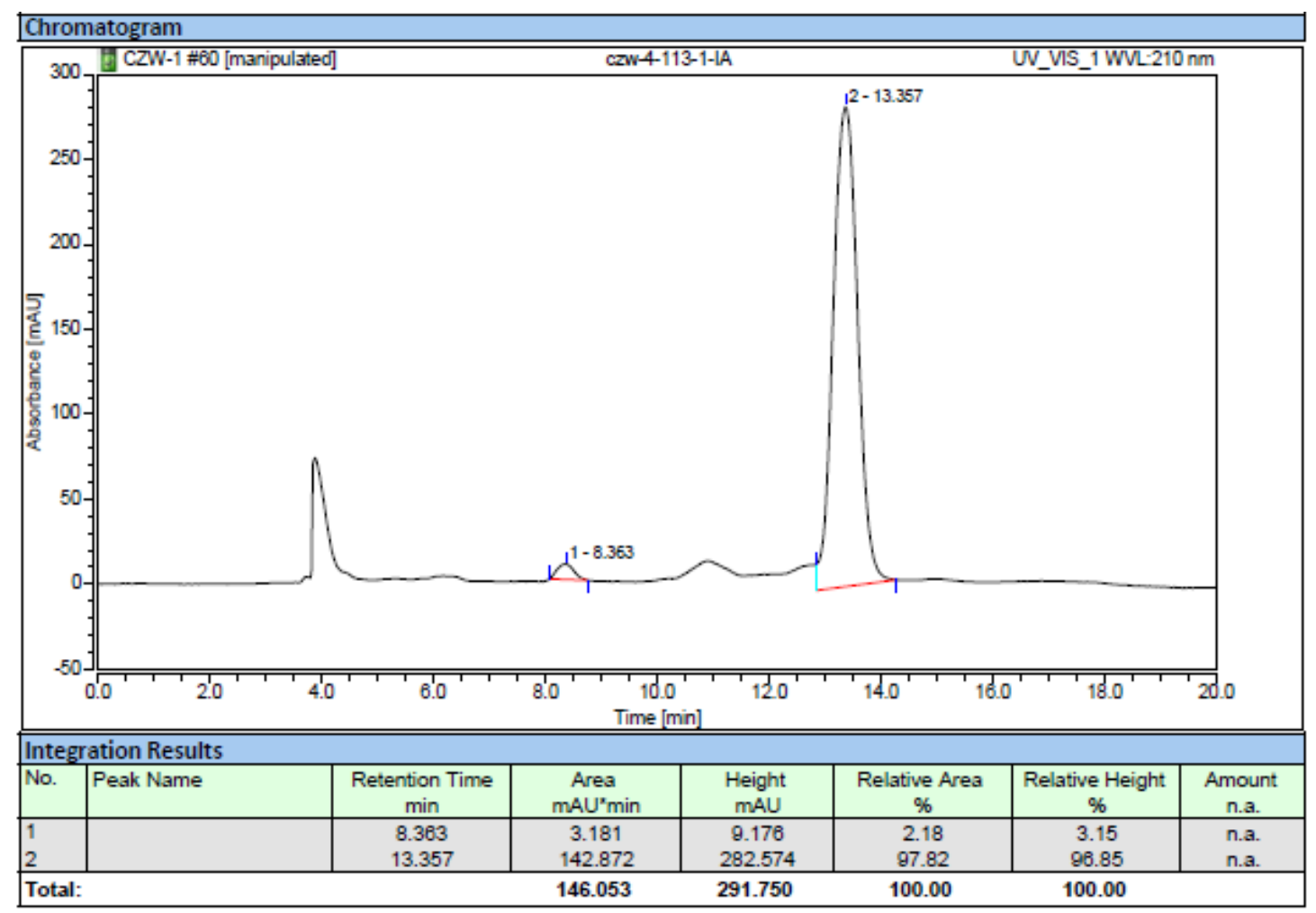

Figure S6: HPLC chromatographs of 3af

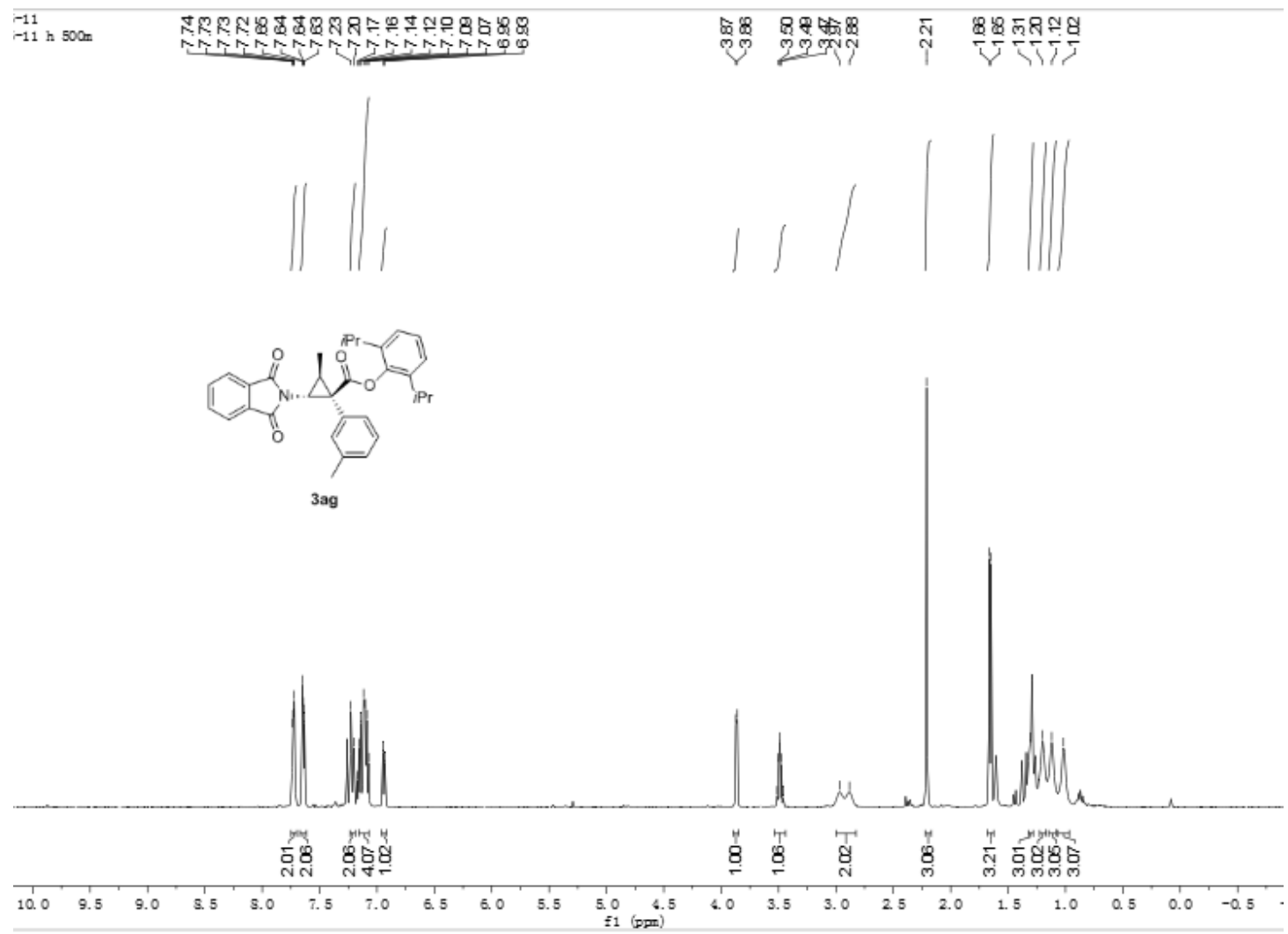

Figure S7: ${ }^{1} \mathrm{H}$ NMR $\left(500 \mathrm{MHz}, \mathrm{CDCl}_{3}\right)$ spectrum for $\mathbf{3 a g}$ 


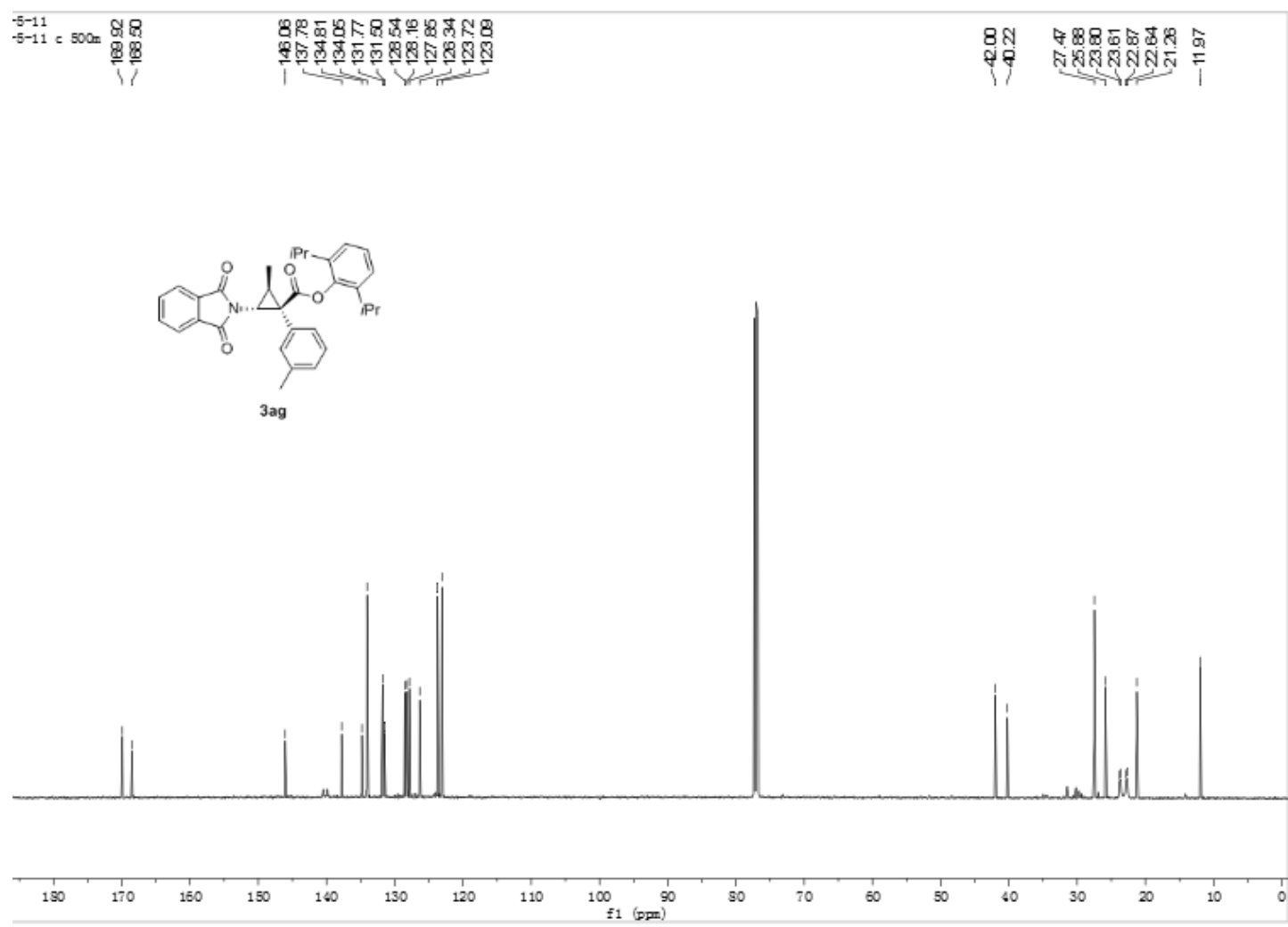

Figure S8: ${ }^{13} \mathrm{C}$ NMR $\left(125 \mathrm{MHz}, \mathrm{CDCl}_{3}\right)$ spectrum for $\mathbf{3 a g}$

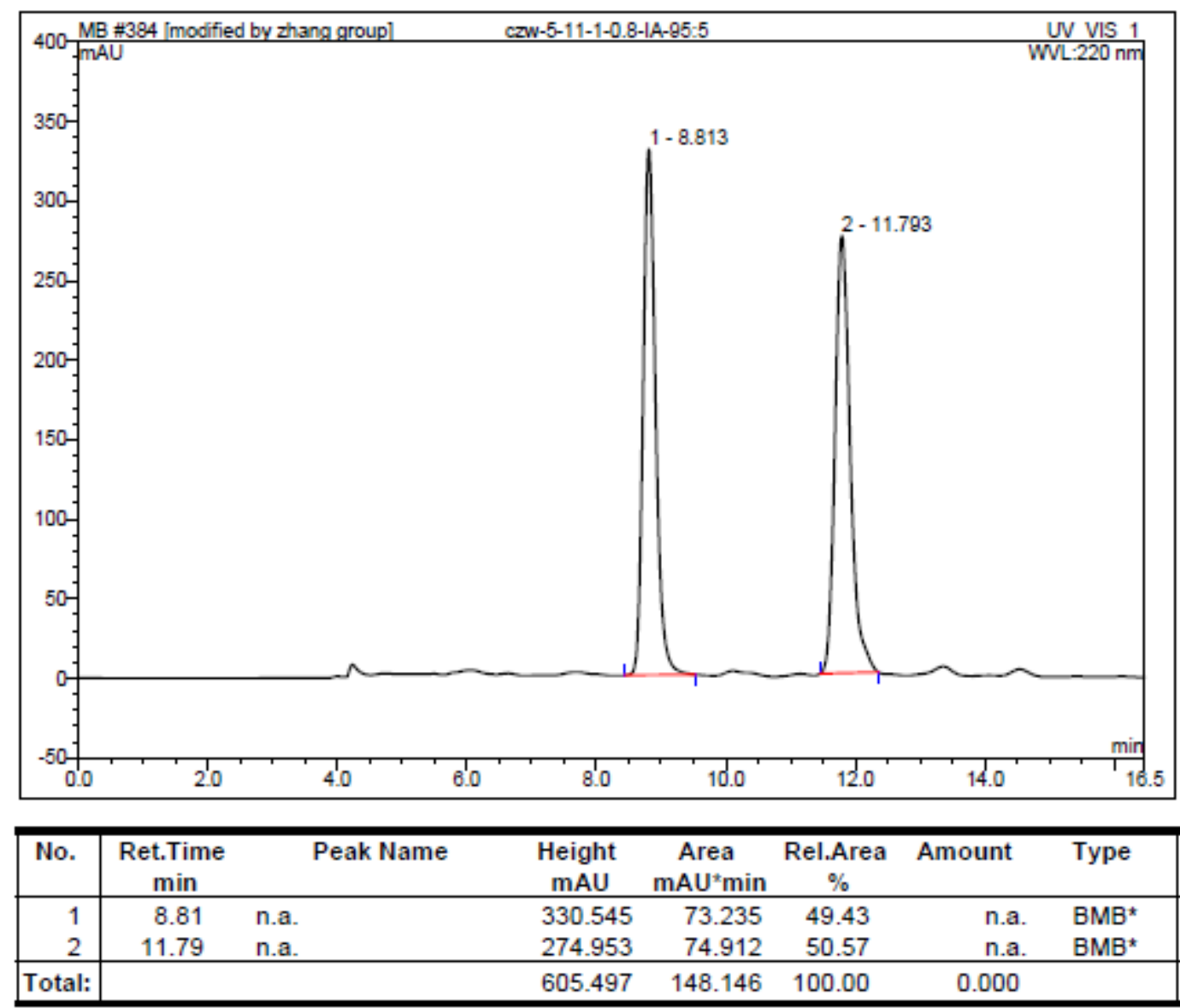




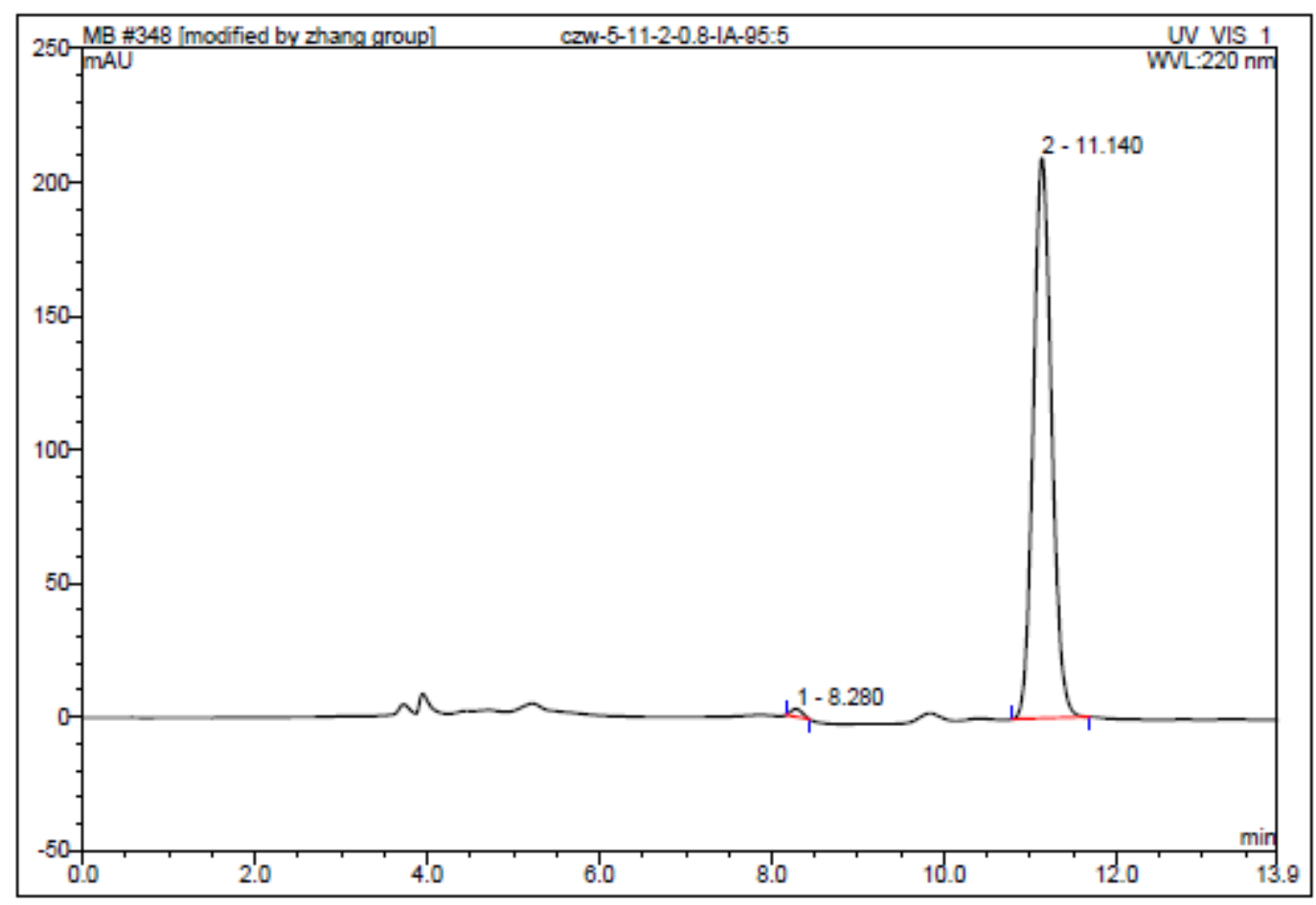

\begin{tabular}{|r|rrrrrrr|}
\hline No. & $\begin{array}{c}\text { Ret.Time } \\
\text { min }\end{array}$ & Peak Name & $\begin{array}{c}\text { Height } \\
\text { mAU }\end{array}$ & $\begin{array}{c}\text { Area } \\
\text { mAU*min }\end{array}$ & $\begin{array}{r}\text { Rel.Area } \\
\%\end{array}$ & Amount & Type \\
\hline 1 & 8.28 & n.a. & 2.908 & 0.438 & 0.84 & n.a. & BMB $^{\star}$ \\
2 & 11.14 & n.a. & 209.521 & 51.635 & 99.16 & n.a. & BMB $^{\star}$ \\
\hline Total: & & & 212.429 & 52.073 & 100.00 & 0.000 & \\
\hline
\end{tabular}

Figure S9: HPLC chromatographs of $\mathbf{3 a g}$

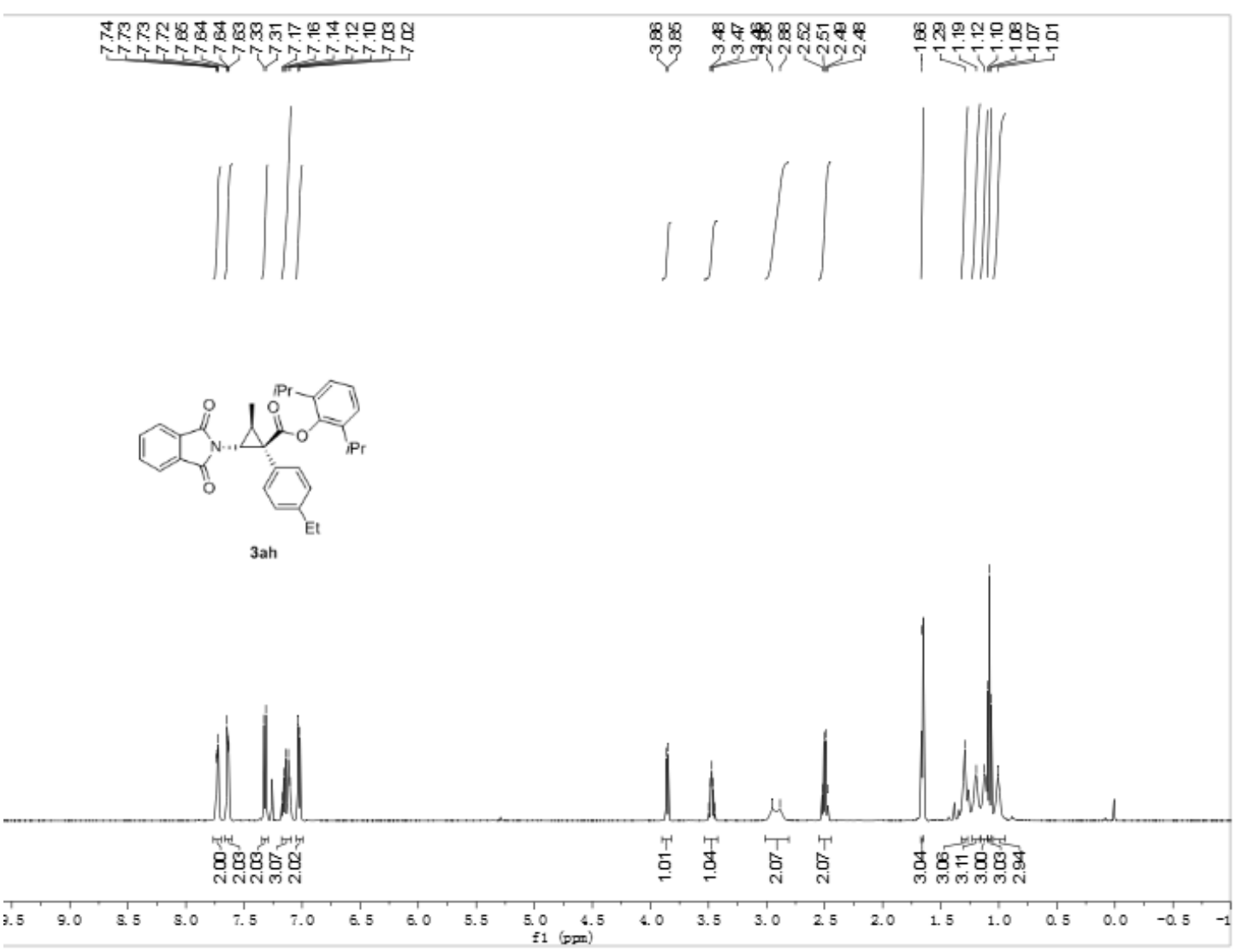


Figure S10: ${ }^{1} \mathrm{H}$ NMR $\left(500 \mathrm{MHz}, \mathrm{CDCl}_{3}\right)$ spectrum for 3ah

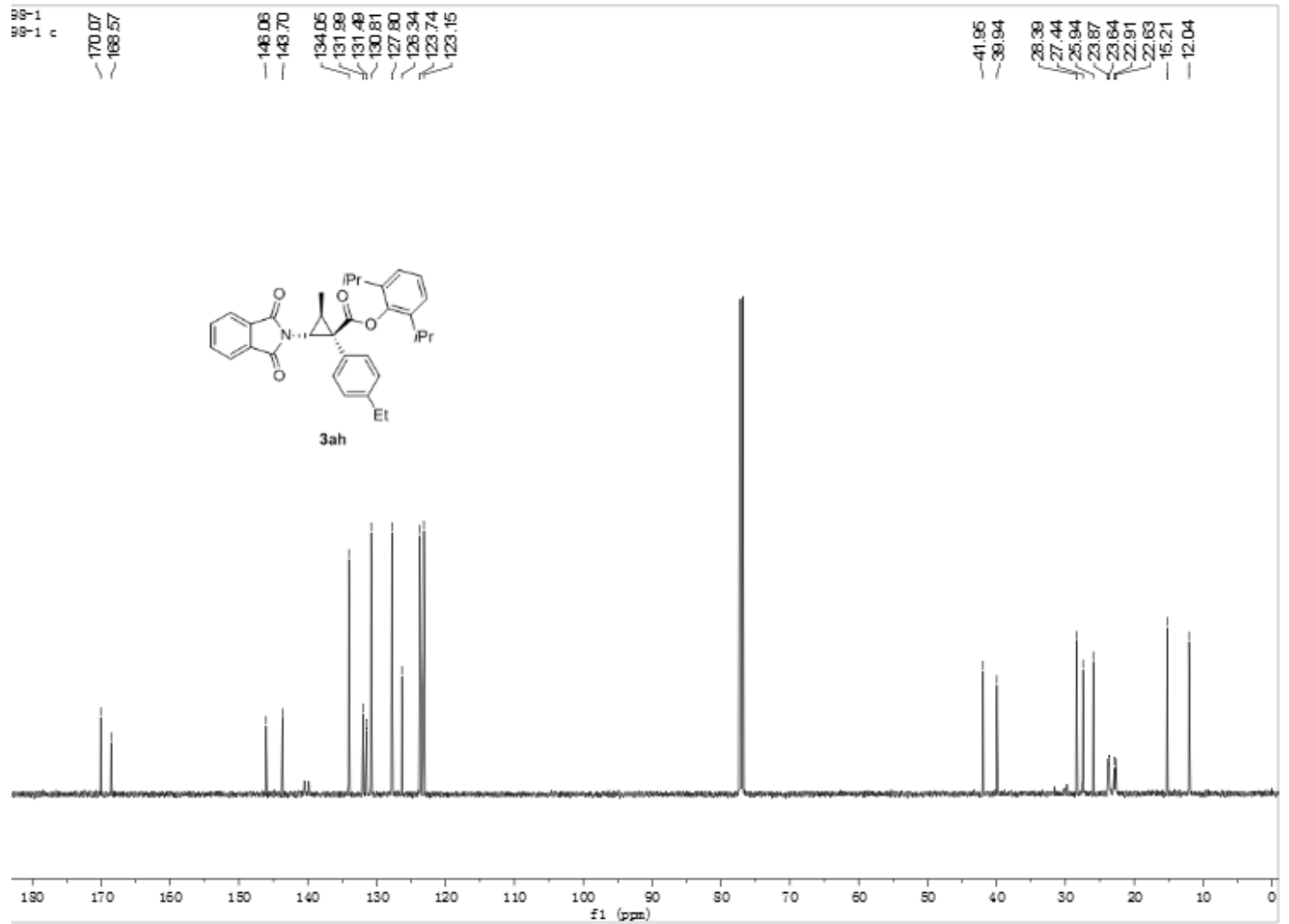

Figure S11: ${ }^{13} \mathrm{C}$ NMR $\left(125 \mathrm{MHz}, \mathrm{CDCl}_{3}\right)$ spectrum for 3ah

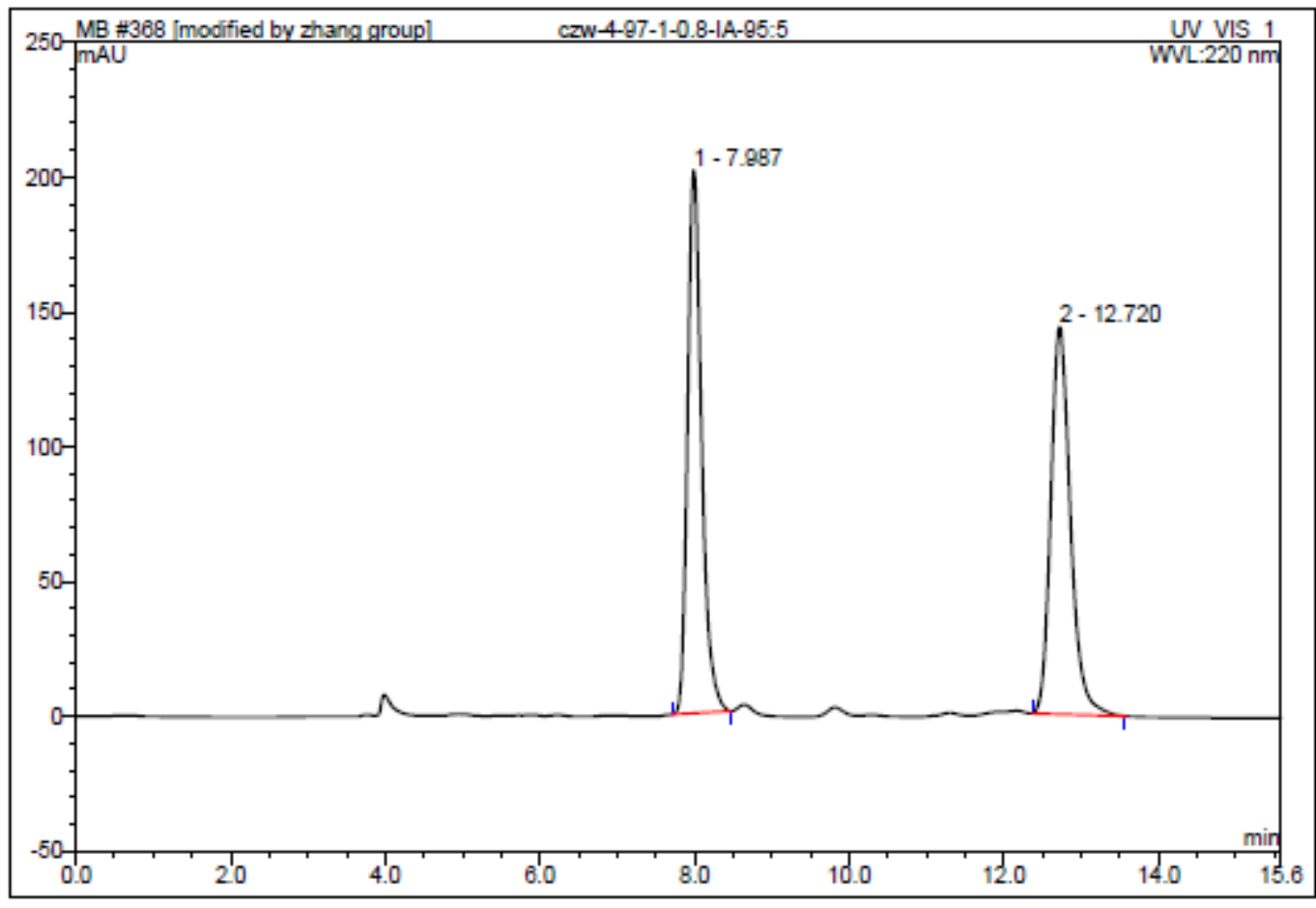

\begin{tabular}{|r|rrrrrrr|}
\hline No. & $\begin{array}{c}\text { Ret.Time } \\
\text { min }\end{array}$ & Peak Name & $\begin{array}{c}\text { Height } \\
\text { mAU }\end{array}$ & $\begin{array}{c}\text { Area } \\
\text { mAU*min }\end{array}$ & $\begin{array}{c}\text { Rel.Area } \\
\%\end{array}$ & Amount & Type \\
\hline 1 & 7.99 & n.a. & 201.528 & 42.348 & 50.02 & n.a. & BMB $^{\star}$ \\
2 & 12.72 & n.a. & 143.713 & 42.313 & 49.98 & n.a. & BMB $^{\star}$ \\
\hline Total: & & & 345.241 & 84.661 & 100.00 & 0.000 & \\
\hline
\end{tabular}




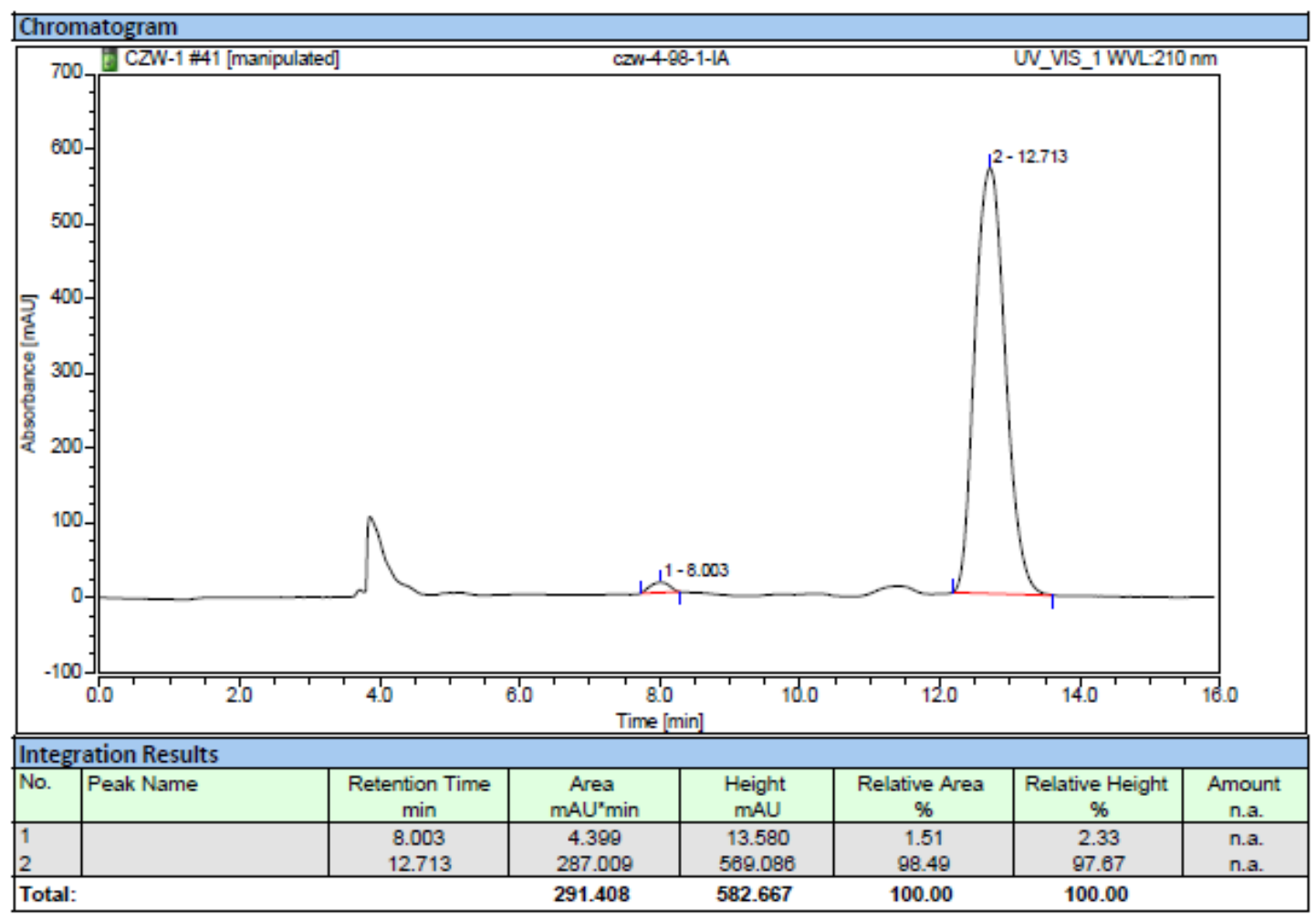

Figure S12: HPLC chromatographs of $\mathbf{3 a h}$

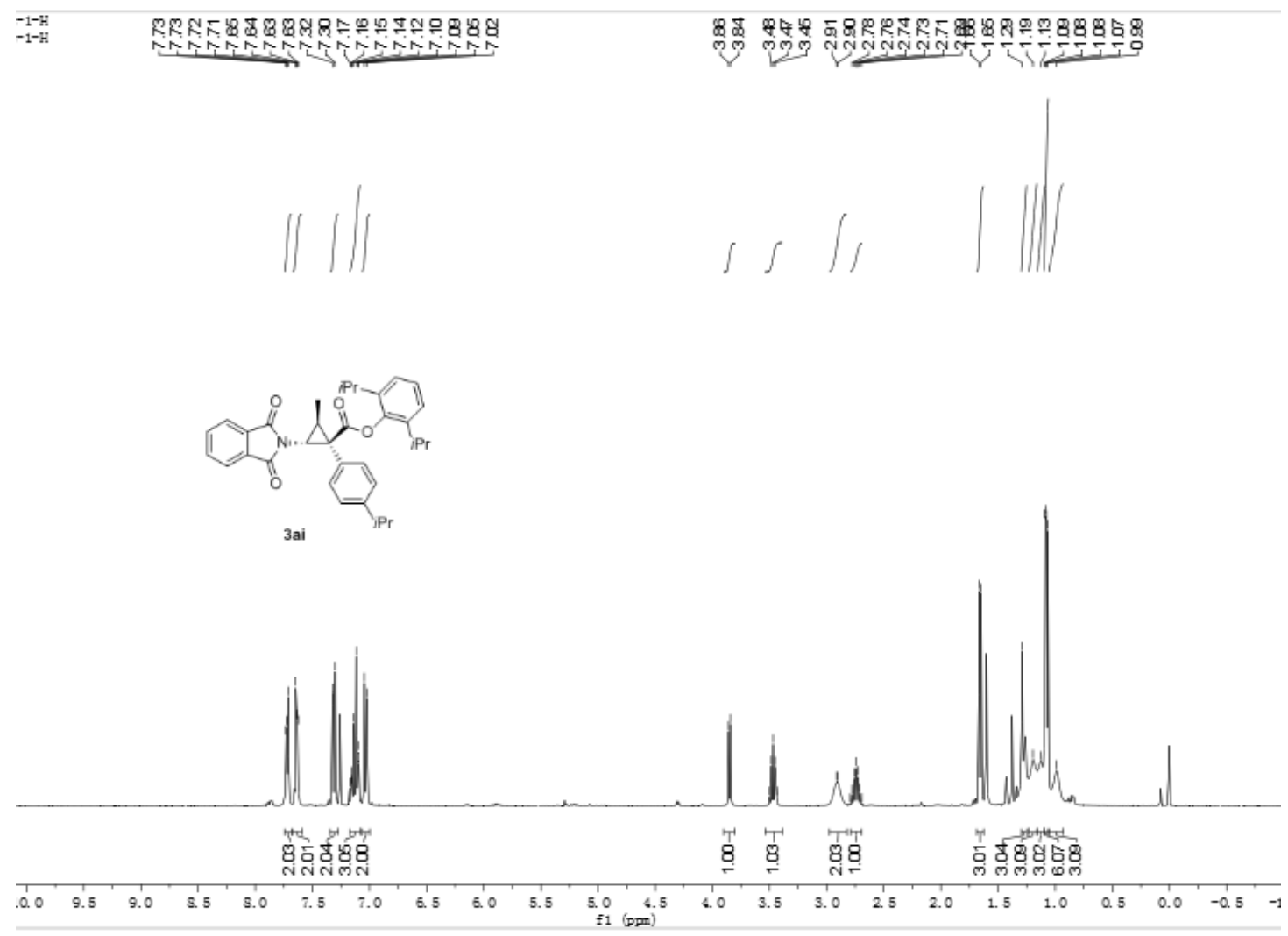

Figure S13: ${ }^{1} \mathrm{H}$ NMR (400 MHz, $\left.\mathrm{CDCl}_{3}\right)$ spectrum for 3ai 


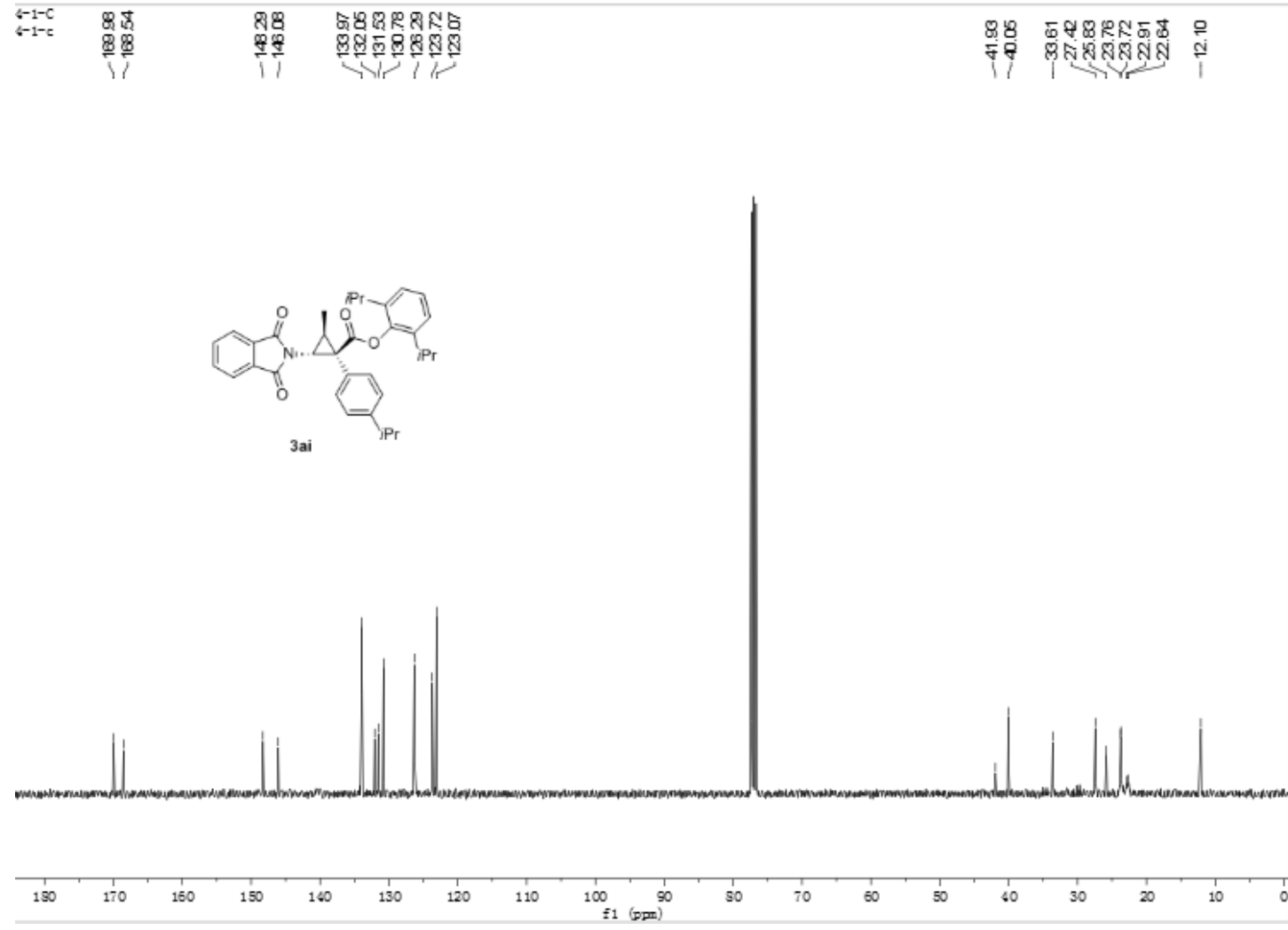

Figure S14: ${ }^{13} \mathrm{C}$ NMR (100 MHz, $\left.\mathrm{CDCl}_{3}\right)$ spectrum for 3ai

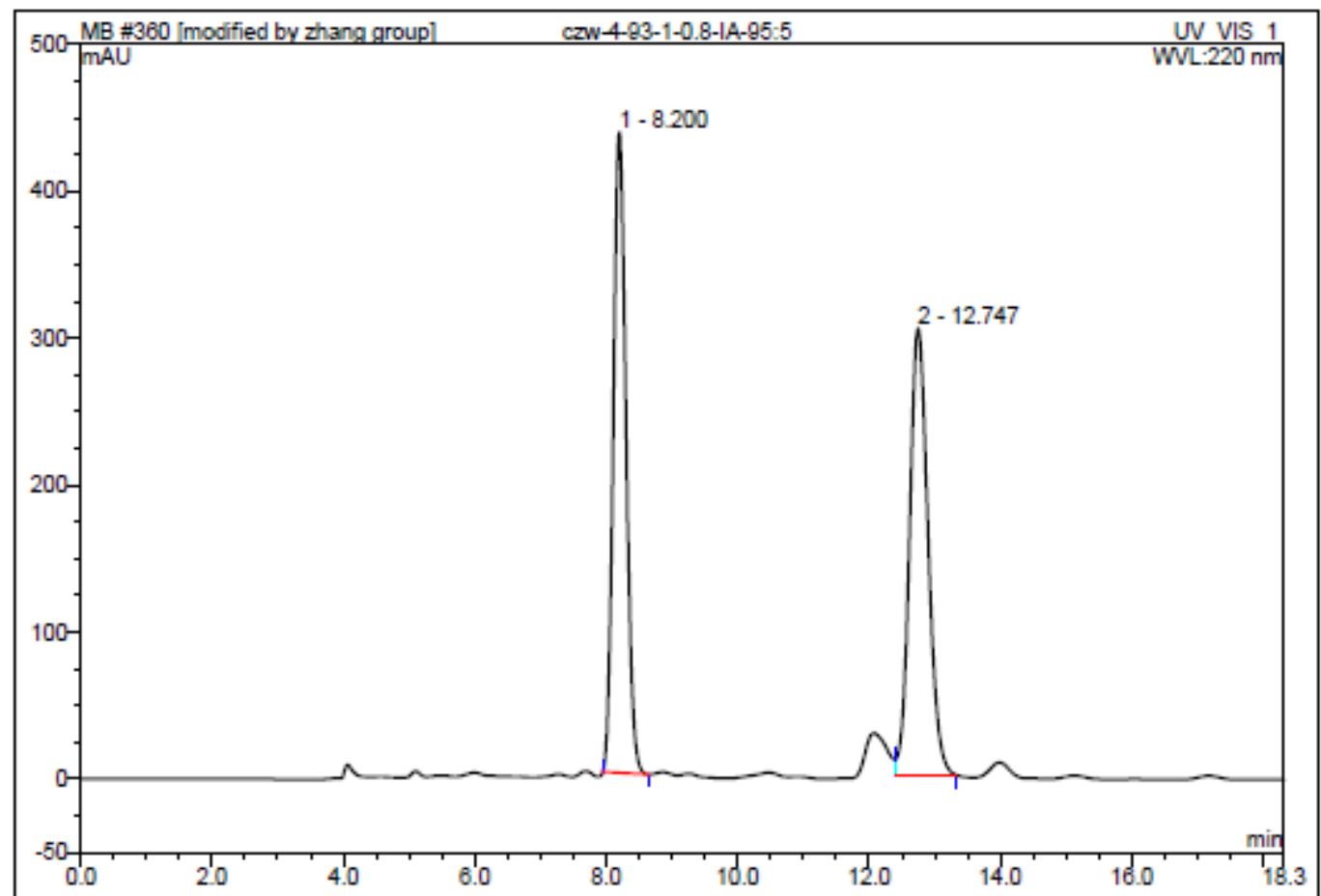

\begin{tabular}{|r|rrrrrrr|}
\hline No. & $\begin{array}{c}\text { Ret.Time } \\
\text { min }\end{array}$ & Peak Name & $\begin{array}{c}\text { Height } \\
\text { mAU }\end{array}$ & $\begin{array}{c}\text { Area } \\
\text { mAU*min }\end{array}$ & $\begin{array}{c}\text { Rel.Area } \\
\%\end{array}$ & Amount & Type \\
\hline 1 & 8.20 & n.a. & 435.844 & 97.140 & 49.58 & n.a. & BMB $^{\star}$ \\
2 & 12.75 & n.a. & 303.637 & 98.802 & 50.42 & n.a. & MB $^{*}$ \\
\hline Total: & & & 739.481 & 195.941 & 100.00 & 0.000 & \\
\hline
\end{tabular}




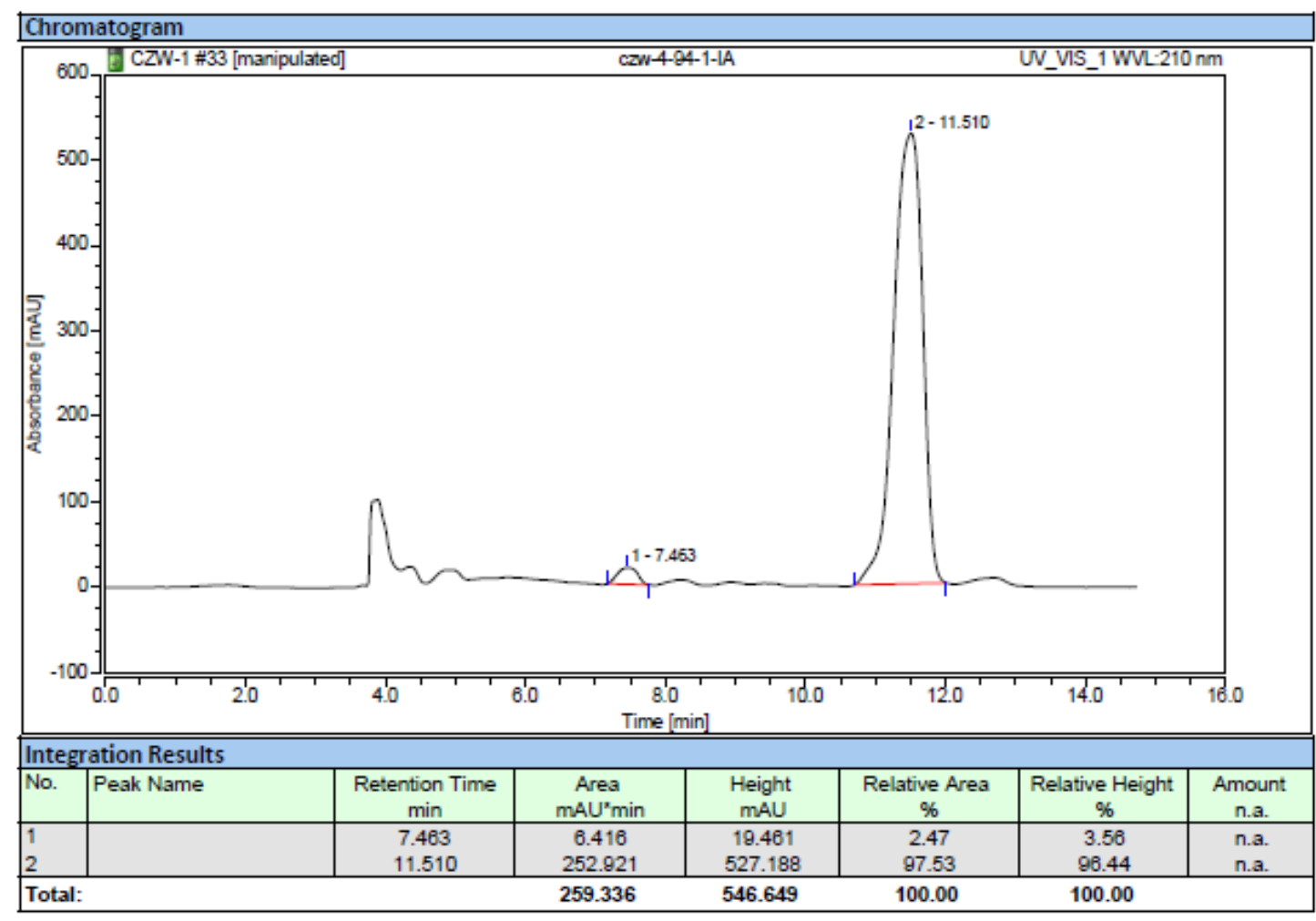

Figure S15: HPLC chromatographs of 3ai

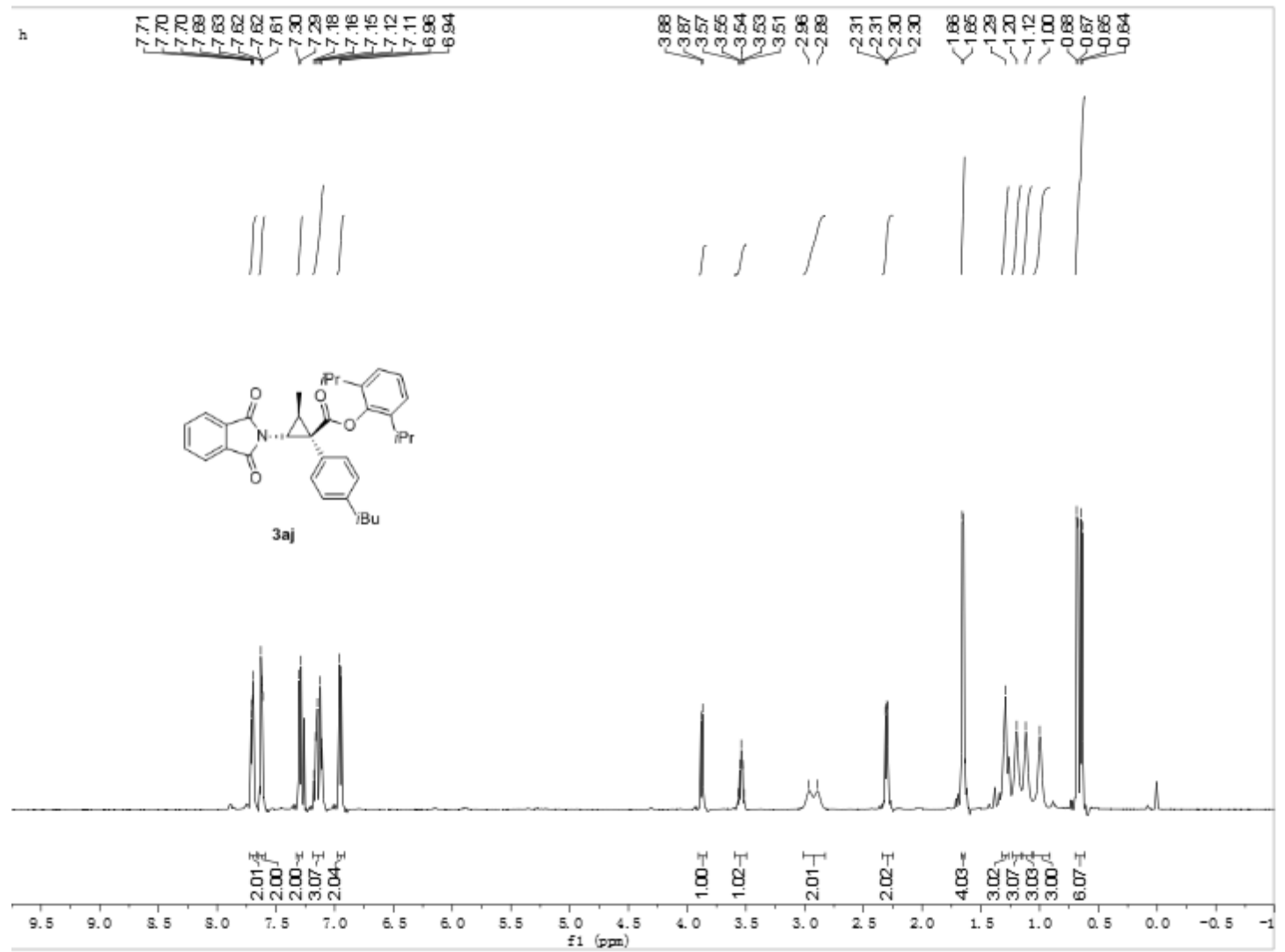

Figure S16: ${ }^{1} \mathrm{H} \mathrm{NMR}\left(500 \mathrm{MHz}, \mathrm{CDCl}_{3}\right)$ spectrum for 3aj 


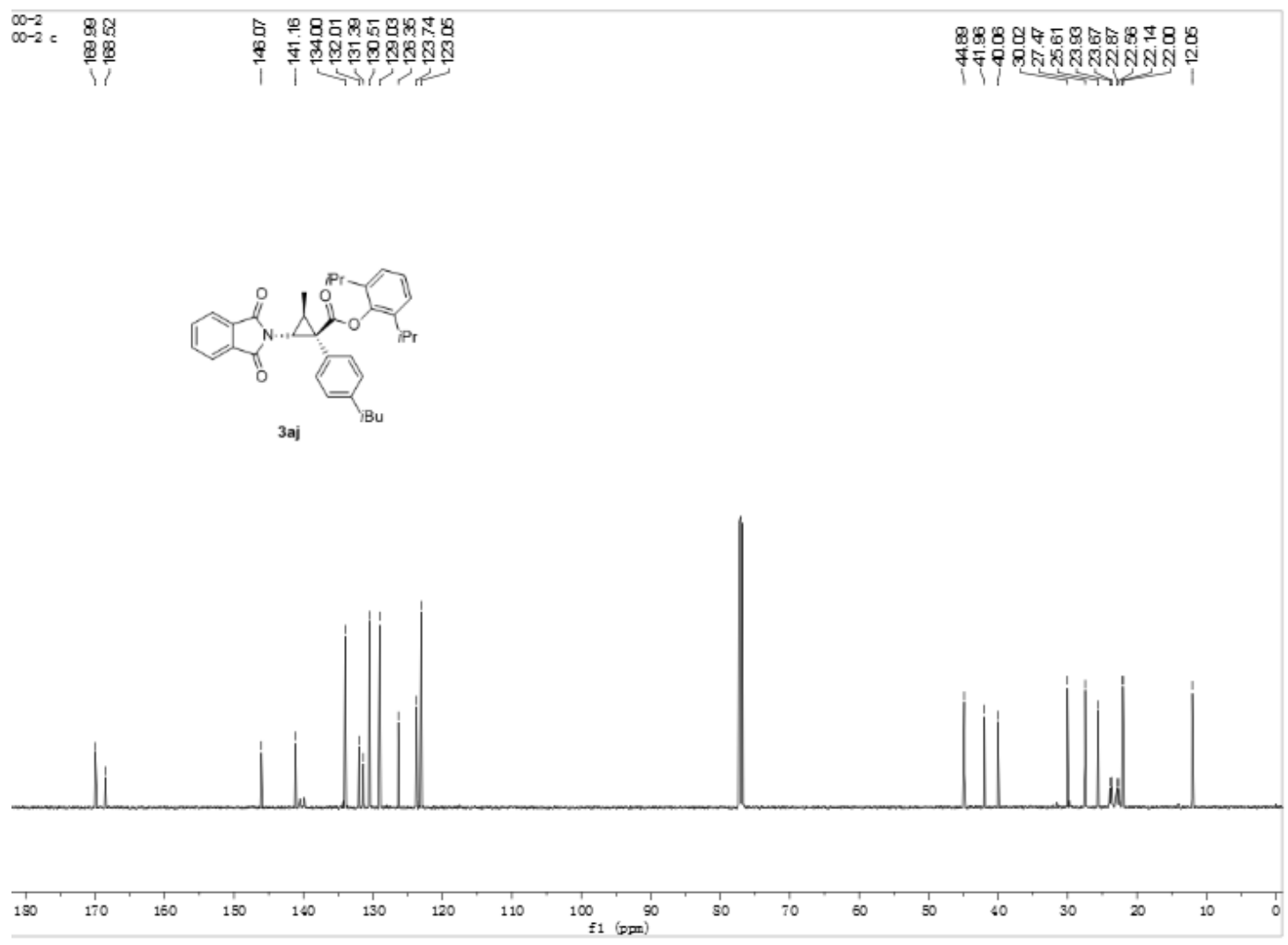

Figure S17: ${ }^{13} \mathrm{C}$ NMR (125 MHz, $\left.\mathrm{CDCl}_{3}\right)$ spectrum for 3aj

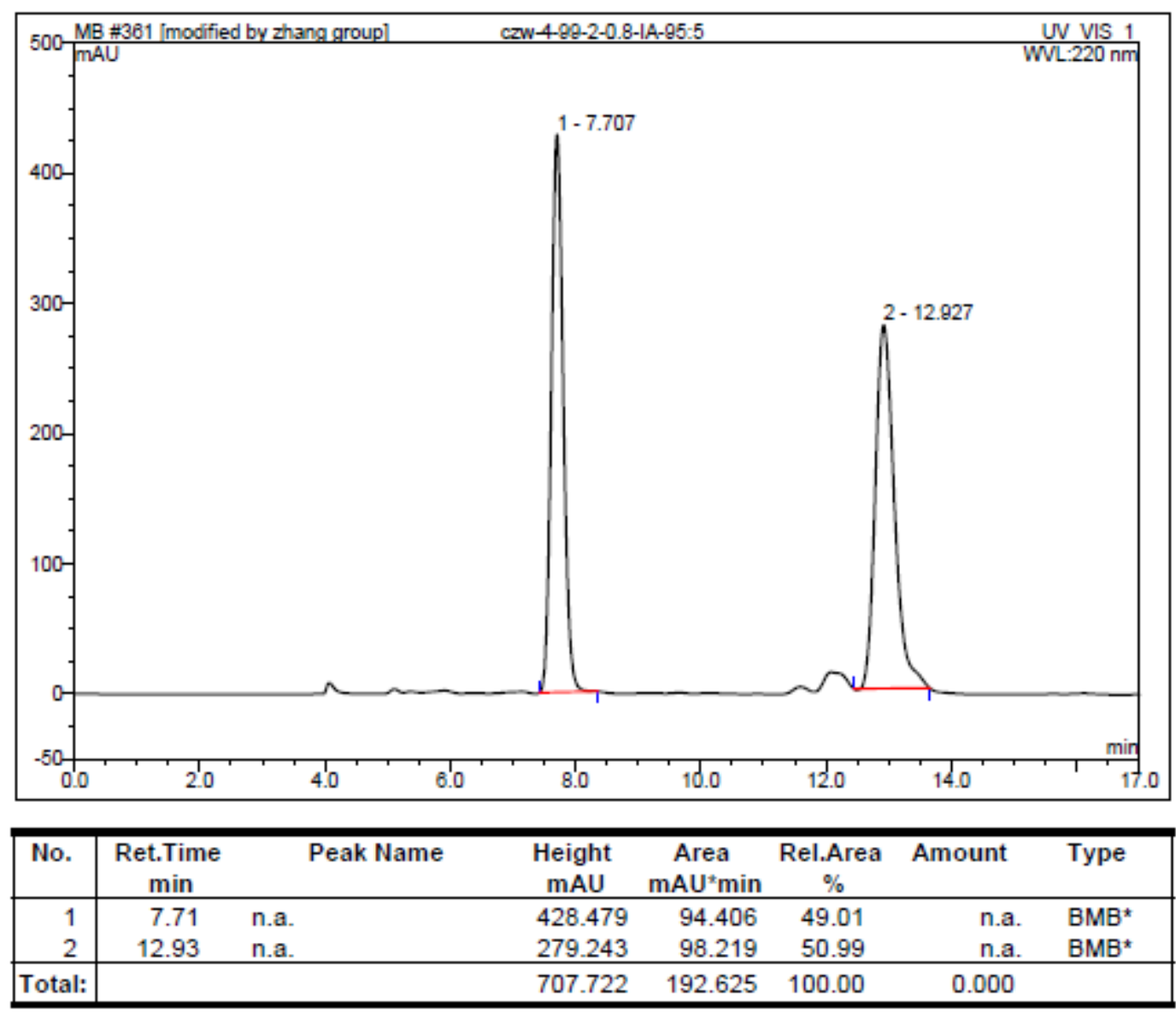




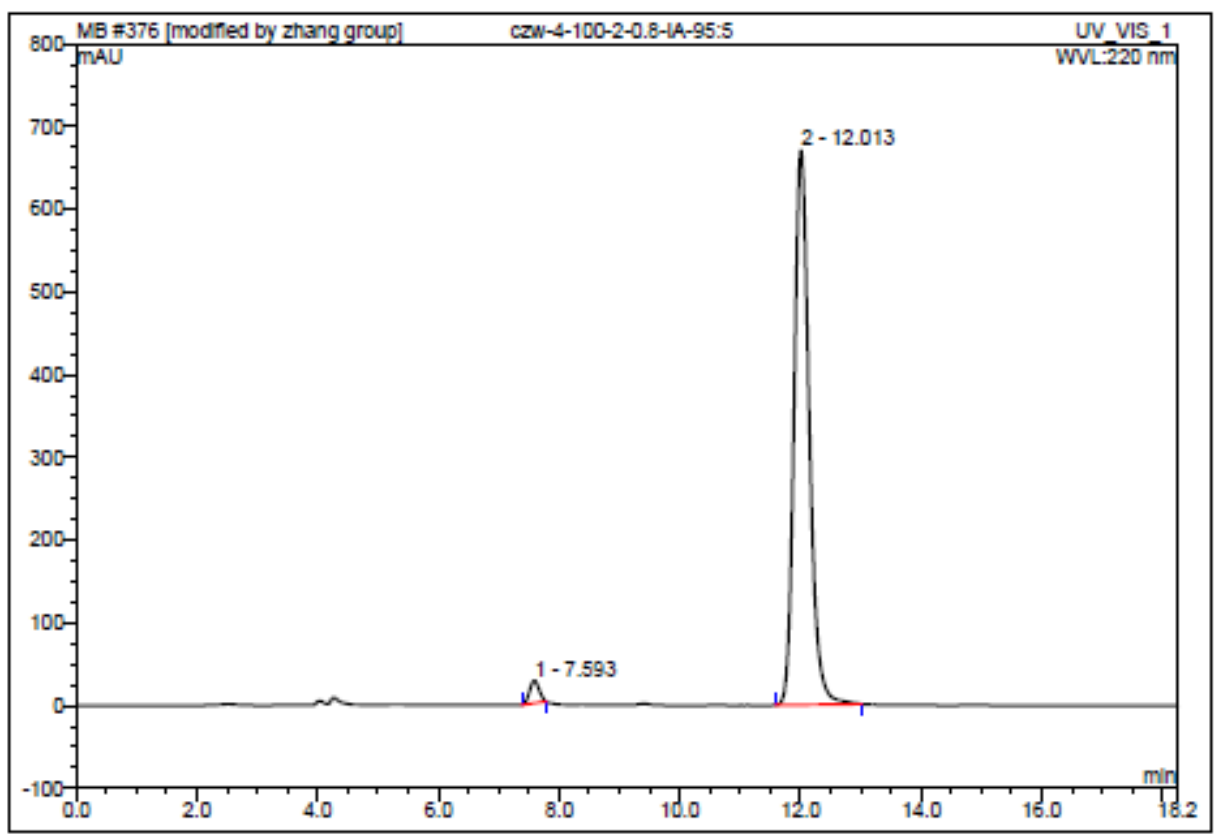

\begin{tabular}{|r|rrrrrrr|}
\hline No. & $\begin{array}{l}\text { Ret.Time } \\
\text { min }\end{array}$ & Peak Name & $\begin{array}{c}\text { Height } \\
\text { mAU }\end{array}$ & $\begin{array}{c}\text { Area } \\
\text { mAU*min }\end{array}$ & $\begin{array}{r}\text { Rel_Area } \\
\%\end{array}$ & Amount & Type \\
\hline 1 & 7.59 & n.a. & 26.943 & 4.900 & 2.45 & n.a. & BMB $^{*}$ \\
2 & 12.01 & n.a. & 670.601 & 194.793 & 97.55 & n.a. & BMB $^{*}$ \\
\hline Total: & & & 697.543 & 199.693 & 100.00 & 0.000 & \\
\hline
\end{tabular}

Figure S18: HPLC chromatographs of 3aj

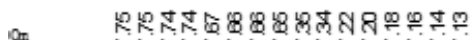

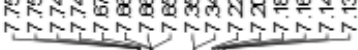

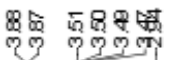

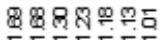

$\int \mid \|$
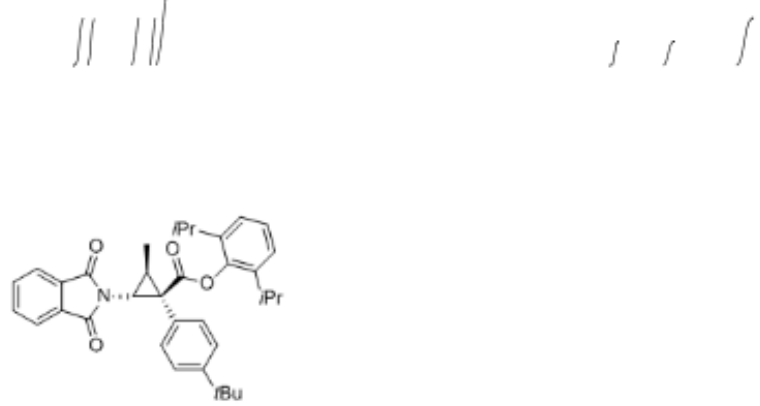

3ak

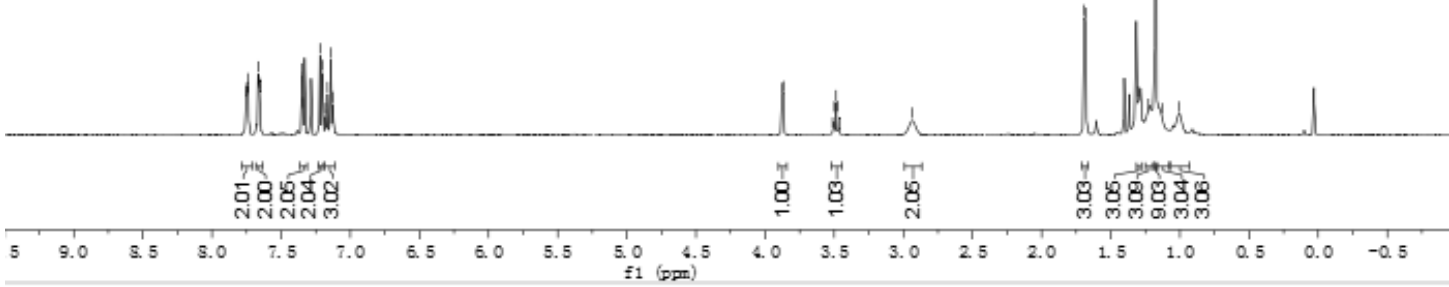

Figure S19: ${ }^{1} \mathrm{H} \mathrm{NMR}\left(500 \mathrm{MHz}, \mathrm{CDCl}_{3}\right)$ spectrum for $\mathbf{3 a k}$ 


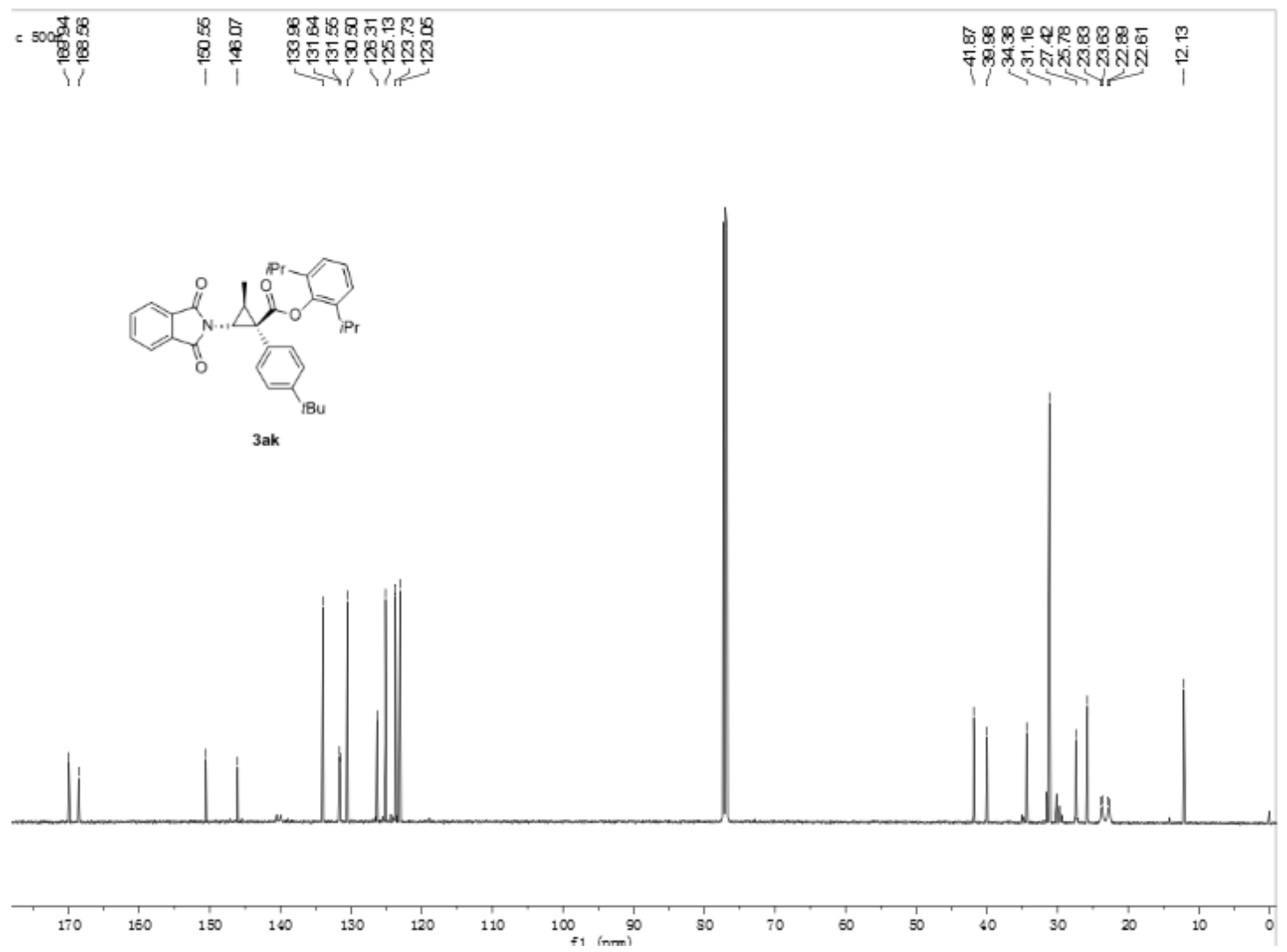

Figure S20: ${ }^{13} \mathrm{C}$ NMR (125 MHz, $\left.\mathrm{CDCl}_{3}\right)$ spectrum for $\mathbf{3 a k}$

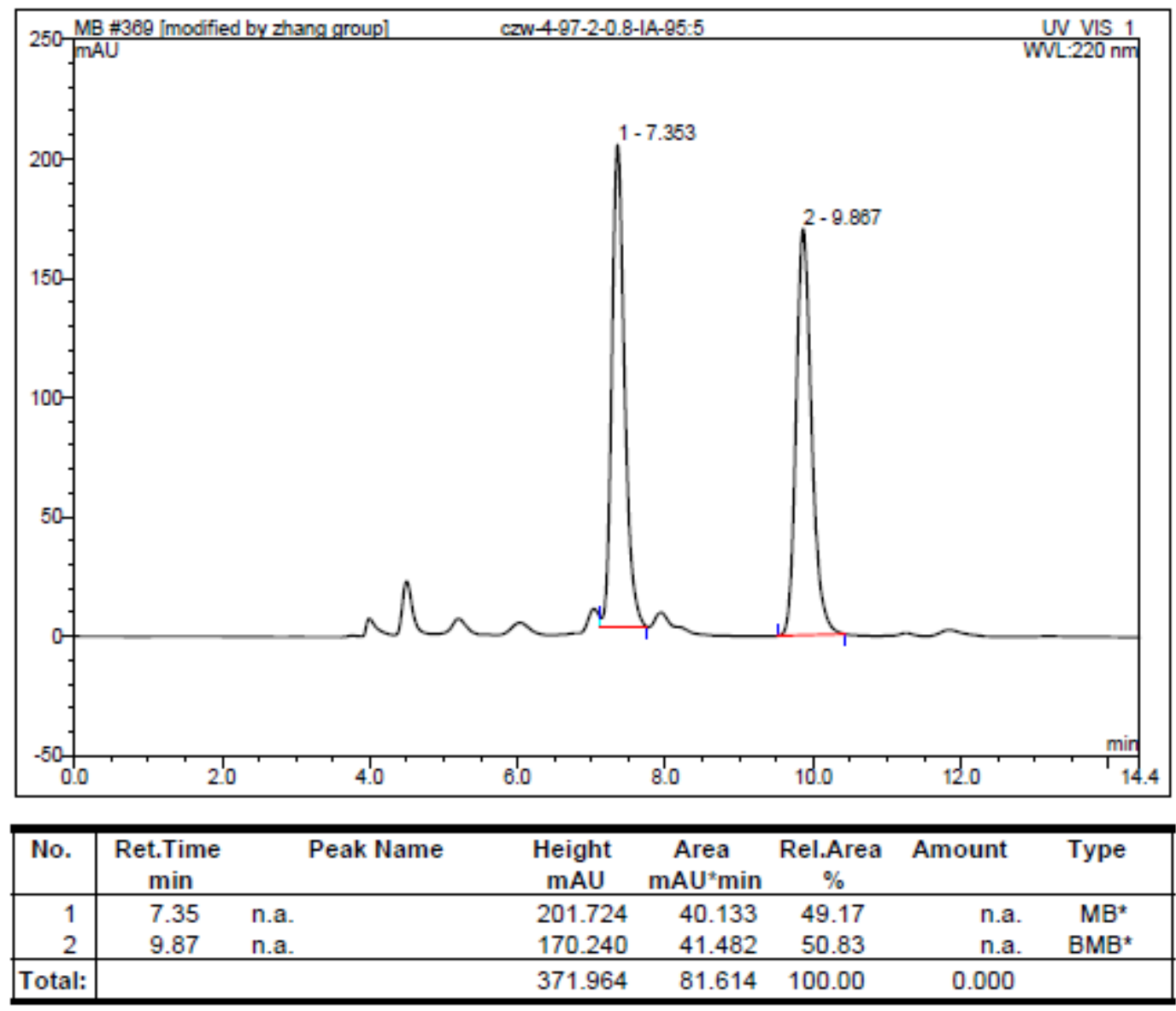




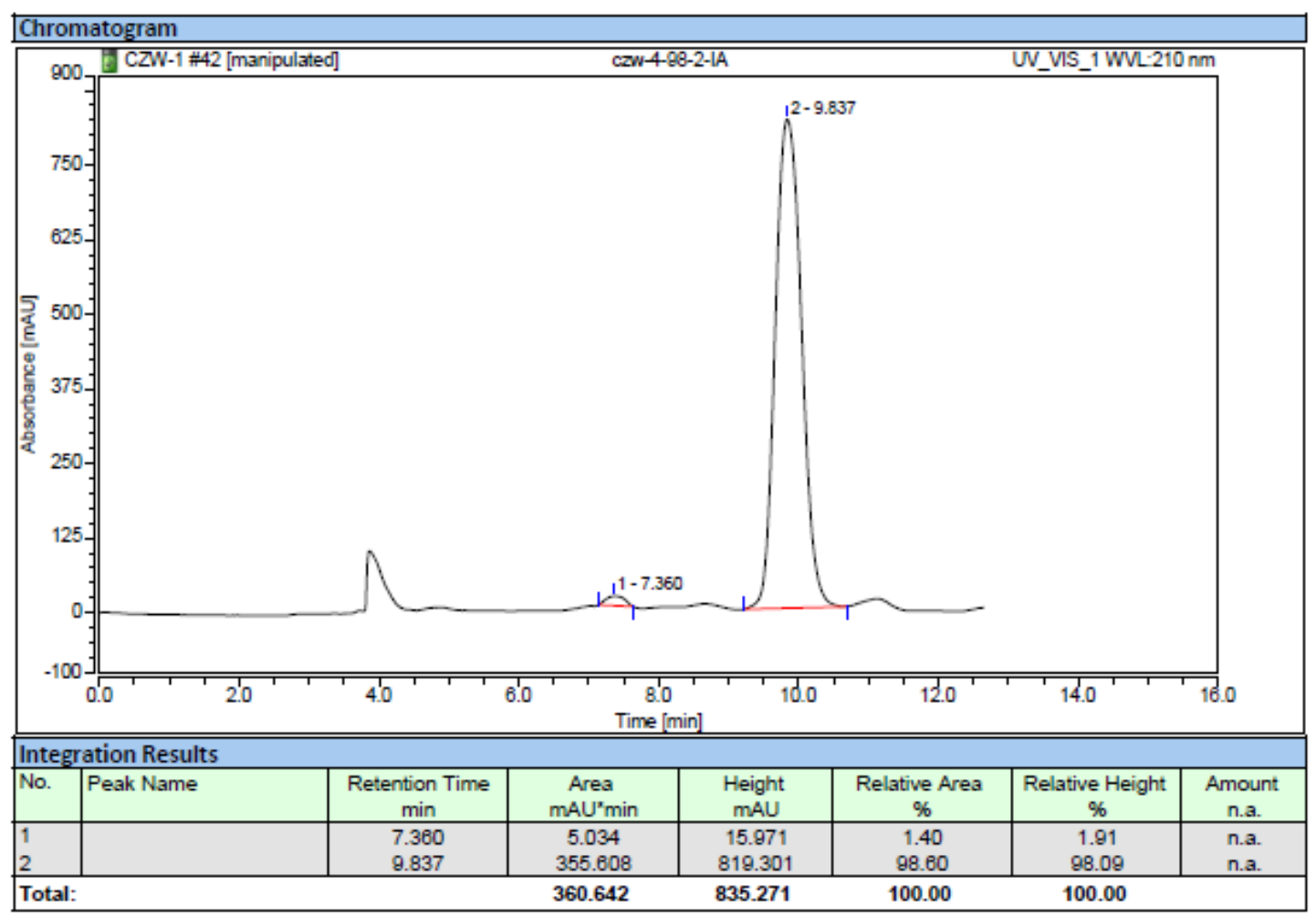

Figure S21: HPLC chromatographs of 3ak

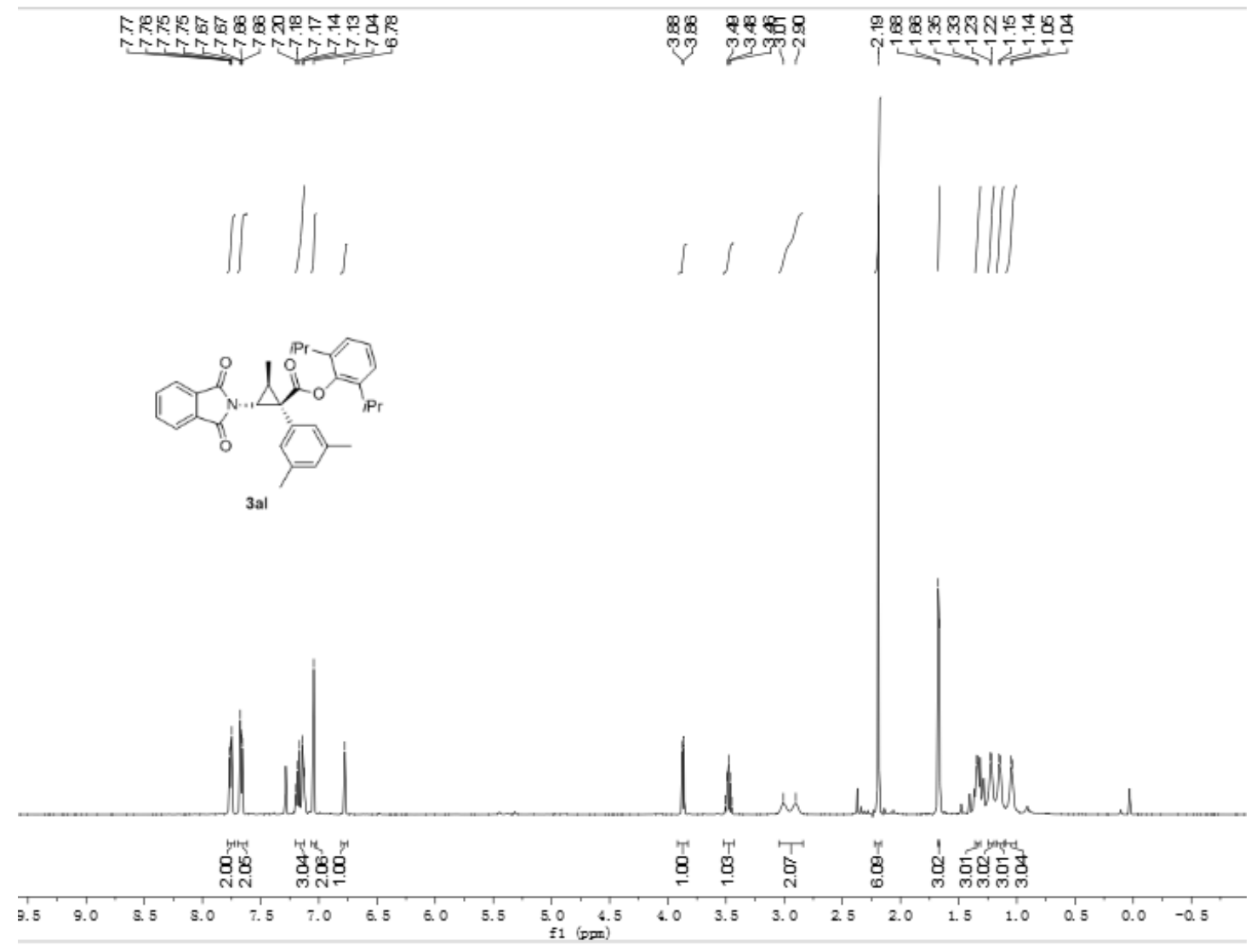

Figure S22: ${ }^{1} \mathrm{H}$ NMR $\left(500 \mathrm{MHz}, \mathrm{CDCl}_{3}\right)$ spectrum for 3al 


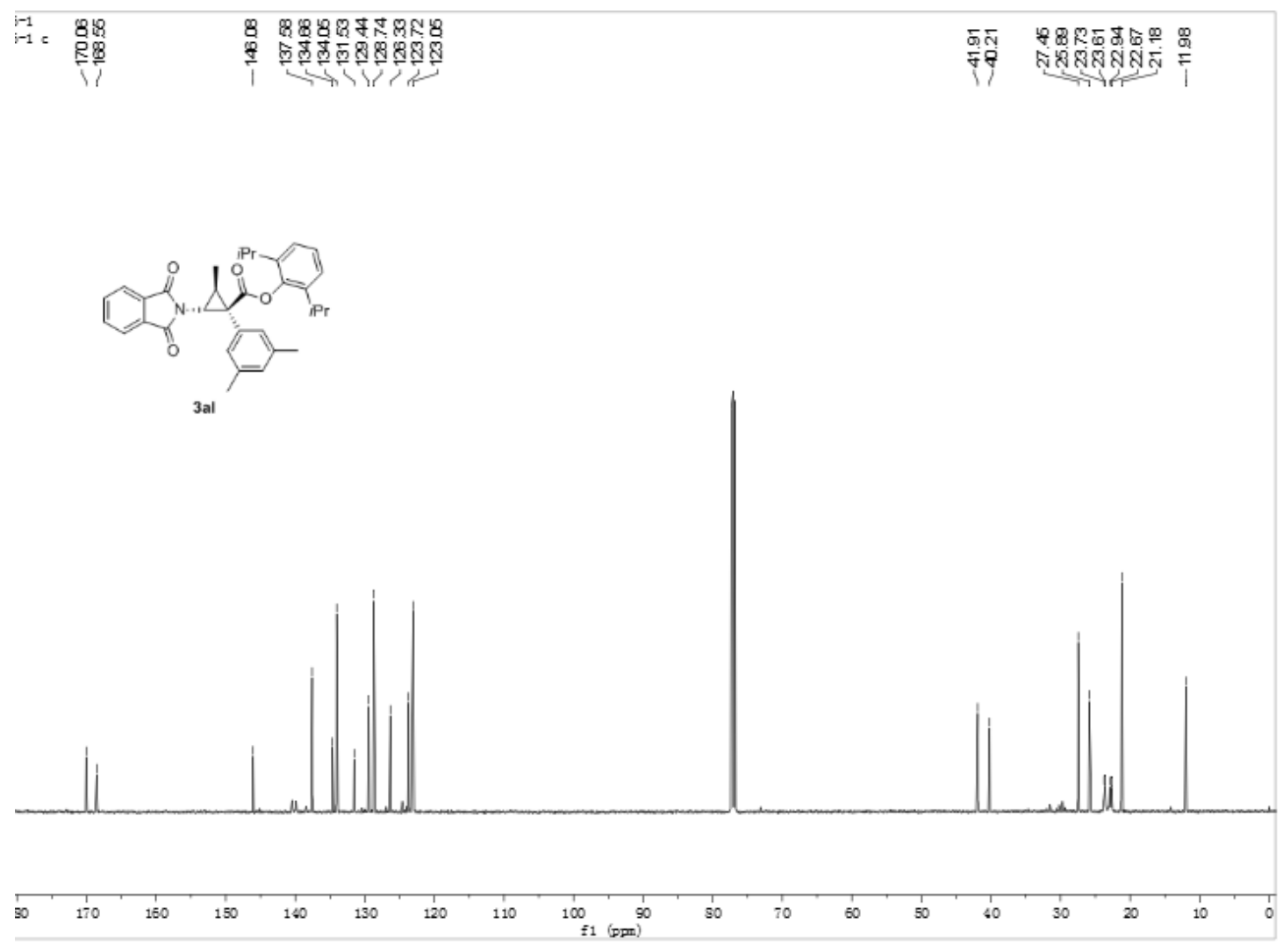

Figure S23: ${ }^{13} \mathrm{C}$ NMR (125 MHz, $\left.\mathrm{CDCl}_{3}\right)$ spectrum for 3al

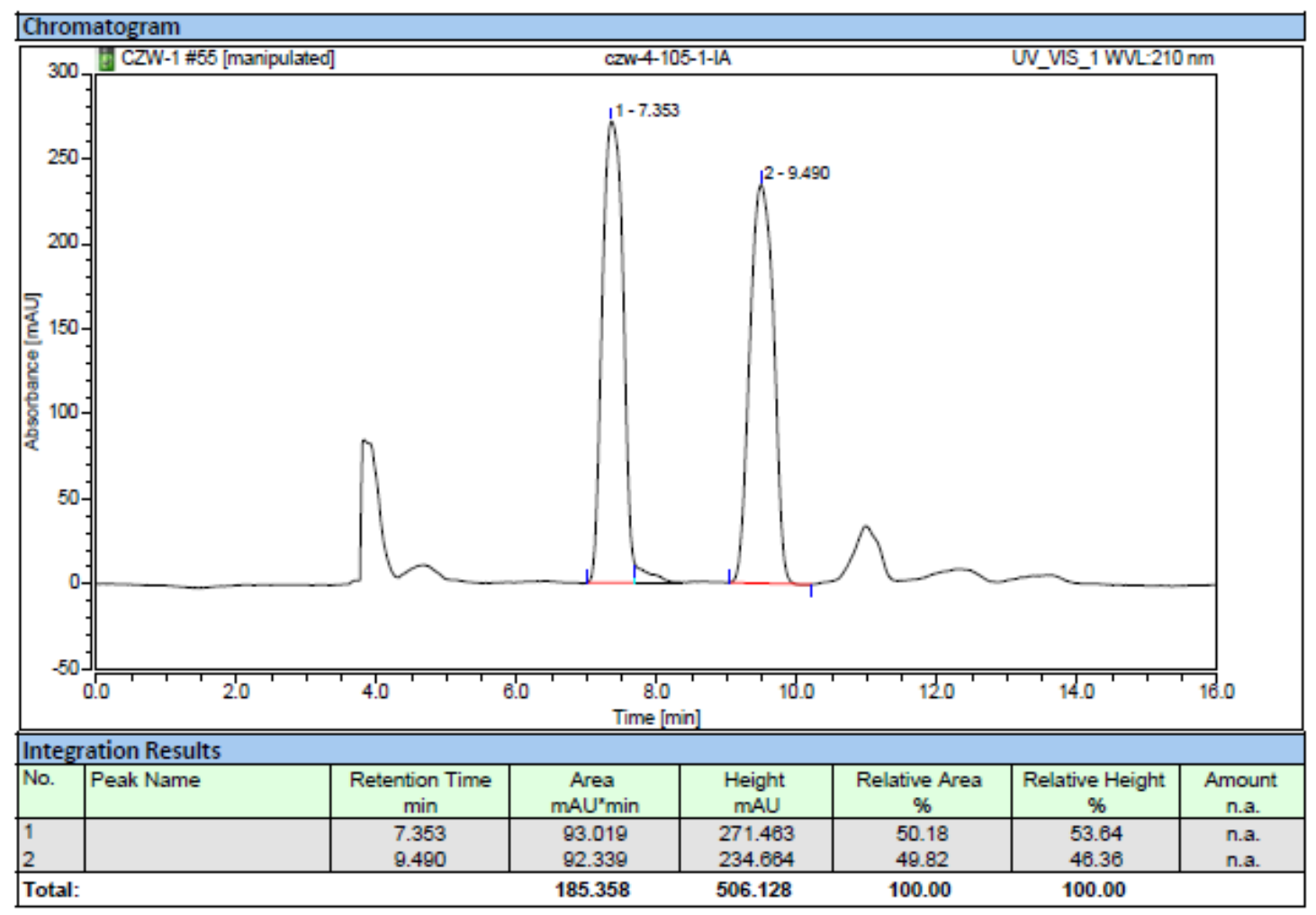




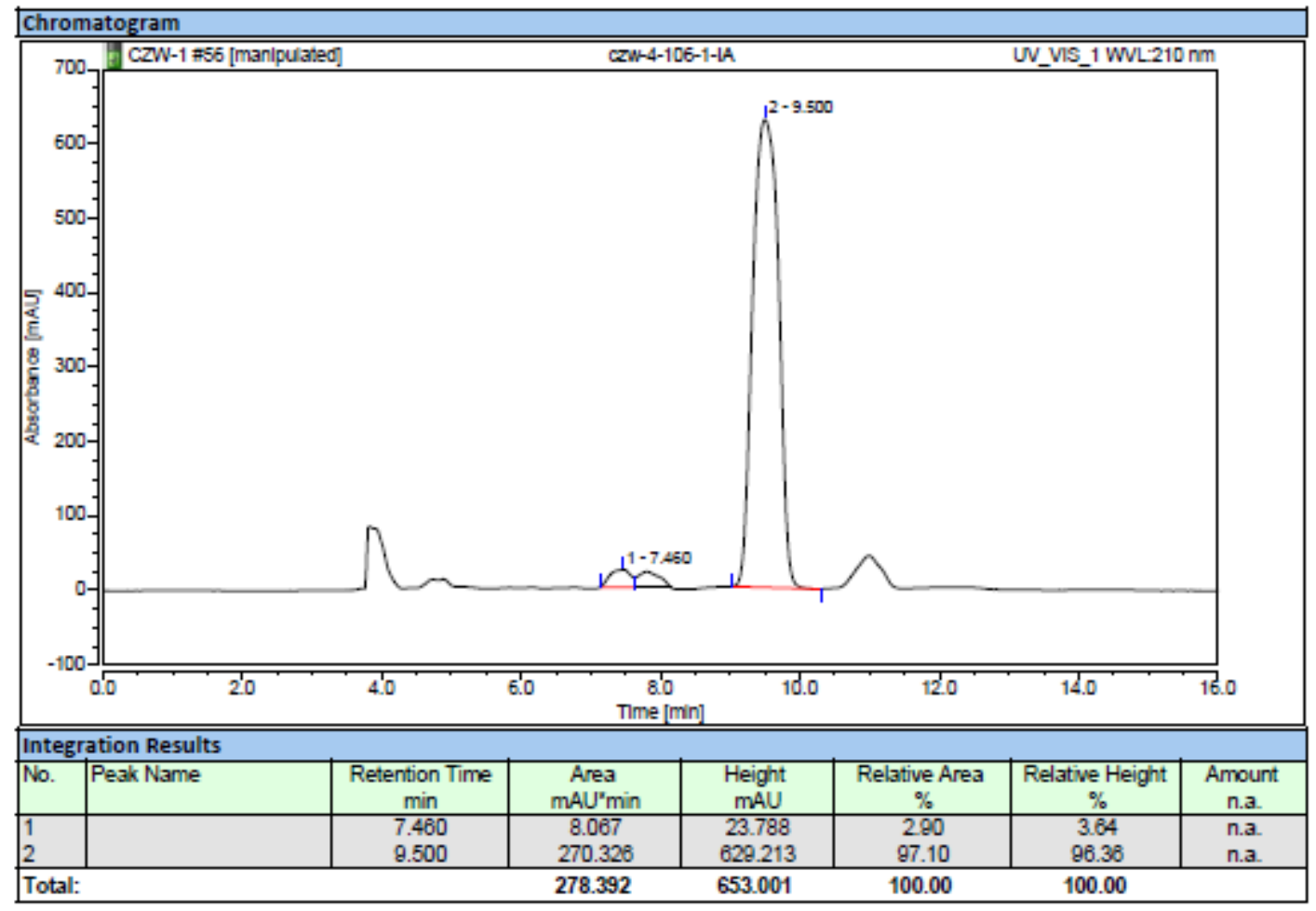

Figure S24: HPLC chromatographs of 3al

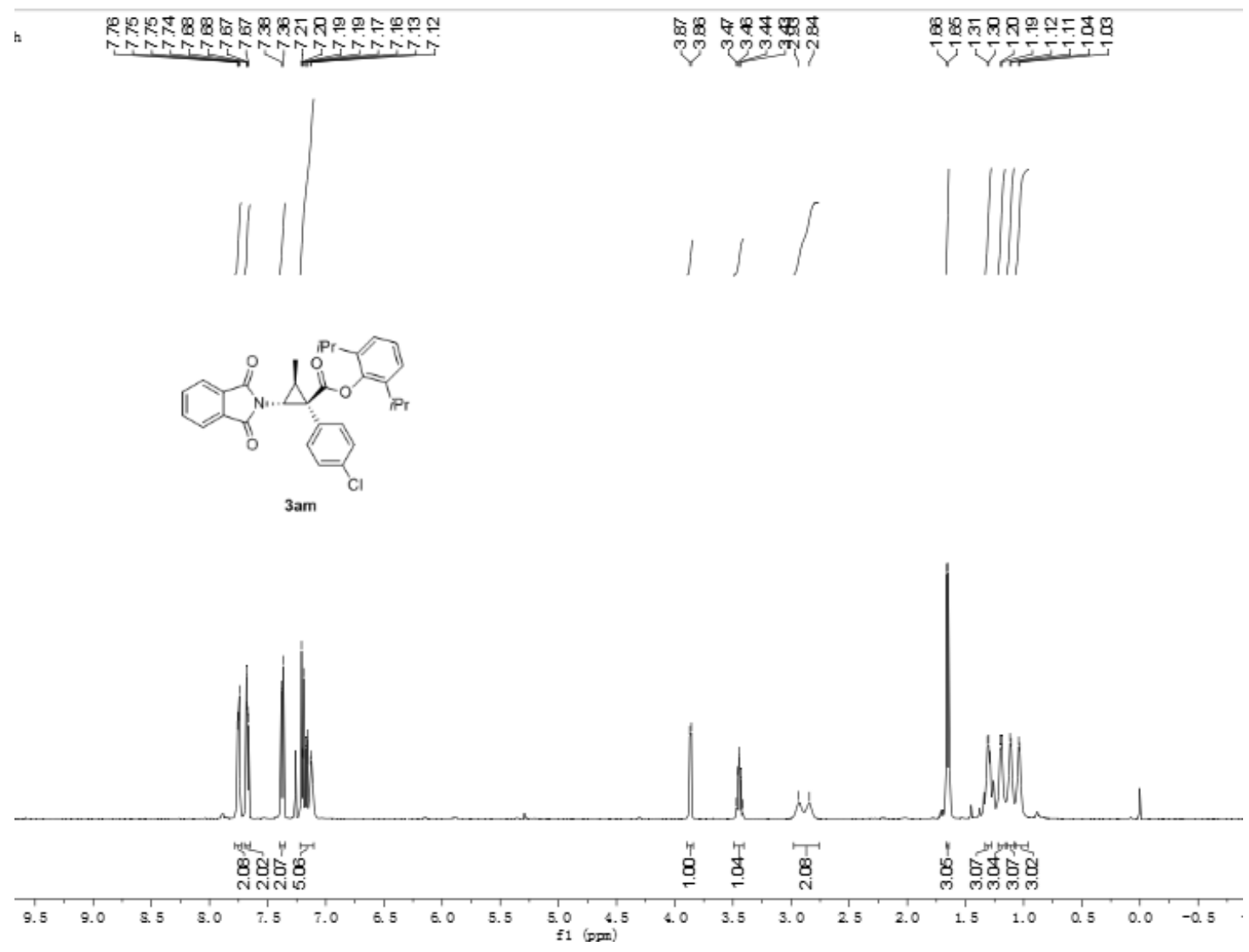

Figure S25: ${ }^{1} \mathrm{H}$ NMR (500 MHz, $\mathrm{CDCl}_{3}$ ) spectrum for 3am 


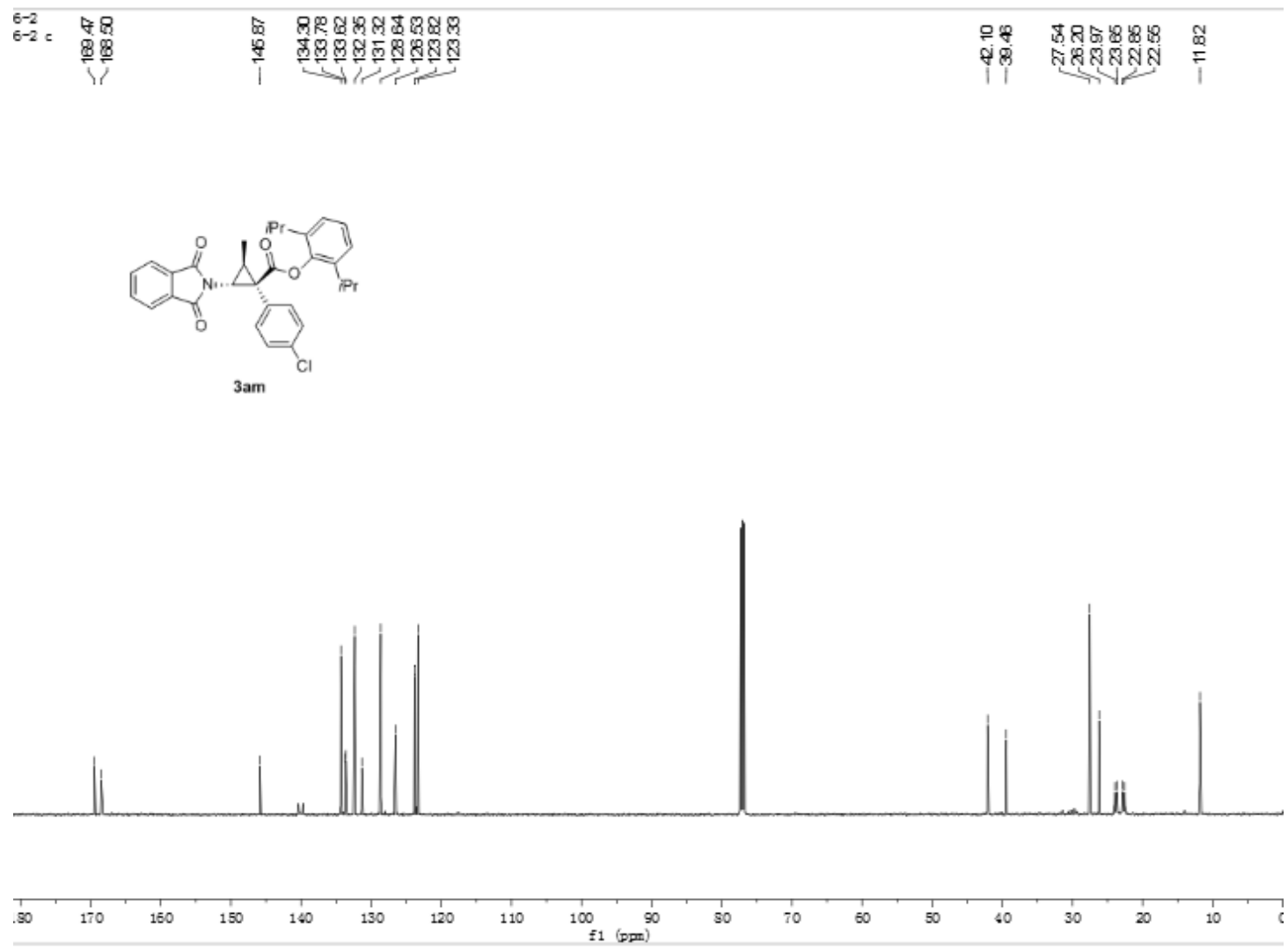

Figure S26: ${ }^{13} \mathrm{C}$ NMR (125 MHz, $\left.\mathrm{CDCl}_{3}\right)$ spectrum for 3am

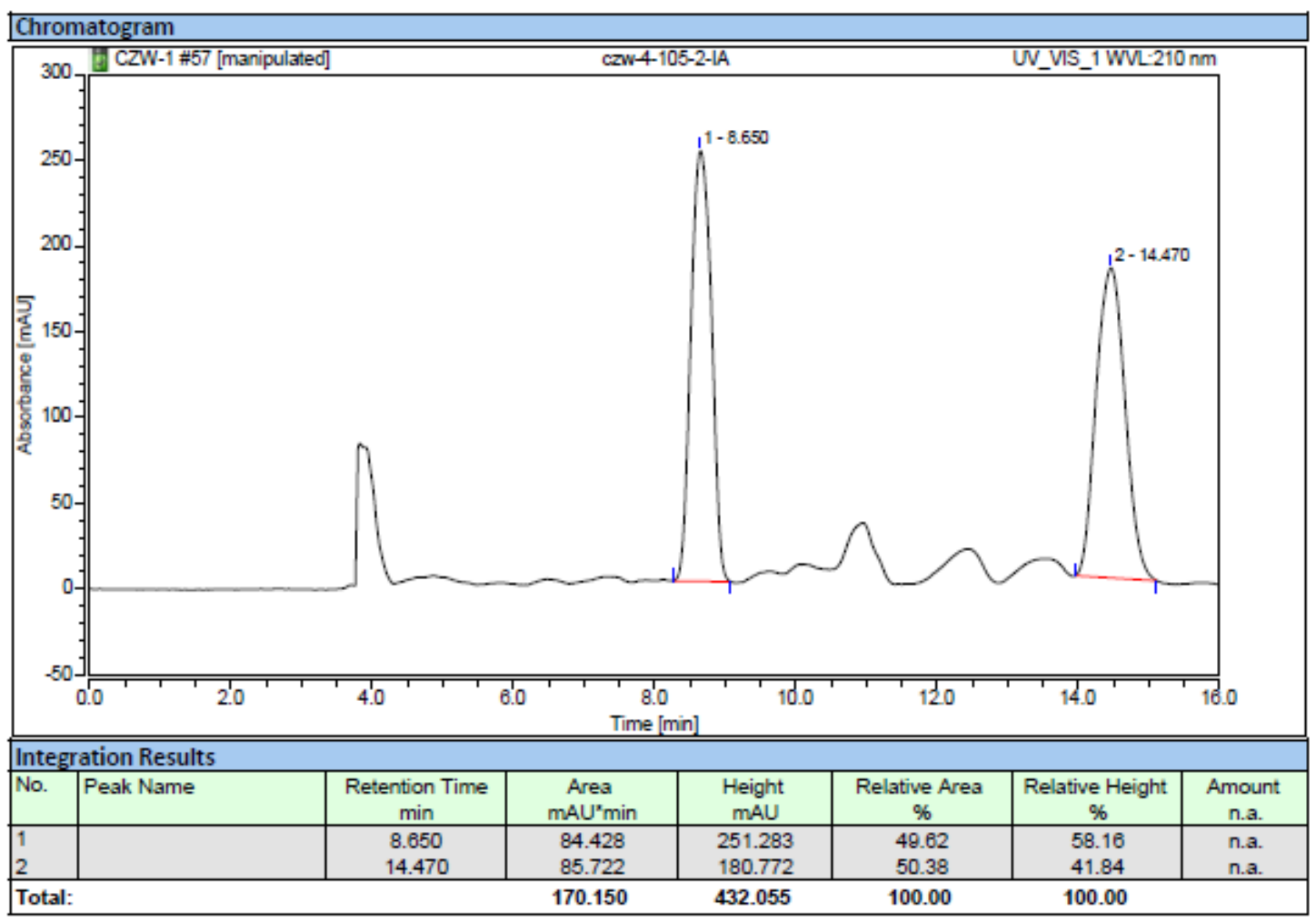




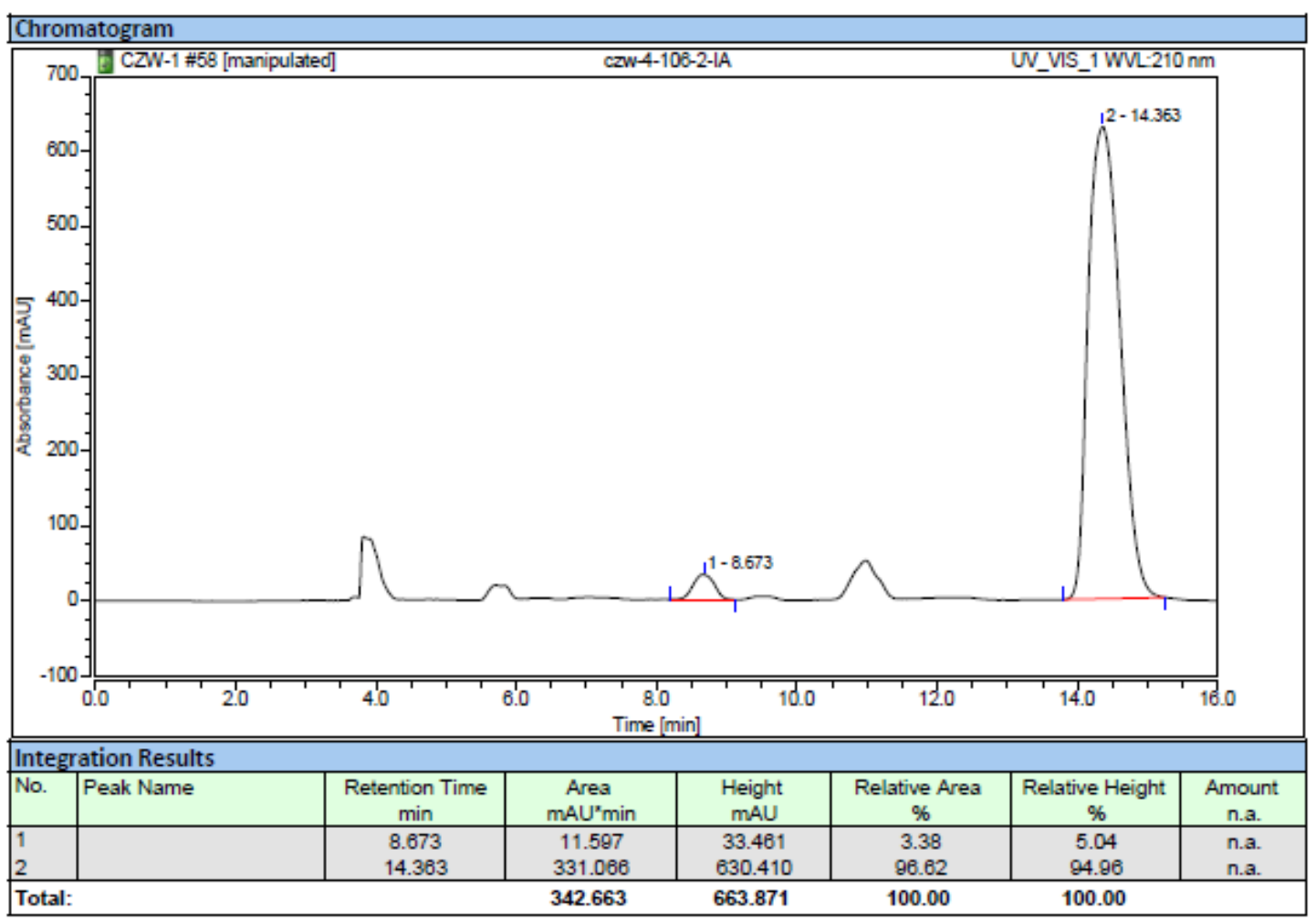

Figure S27: HPLC chromatographs of 3am

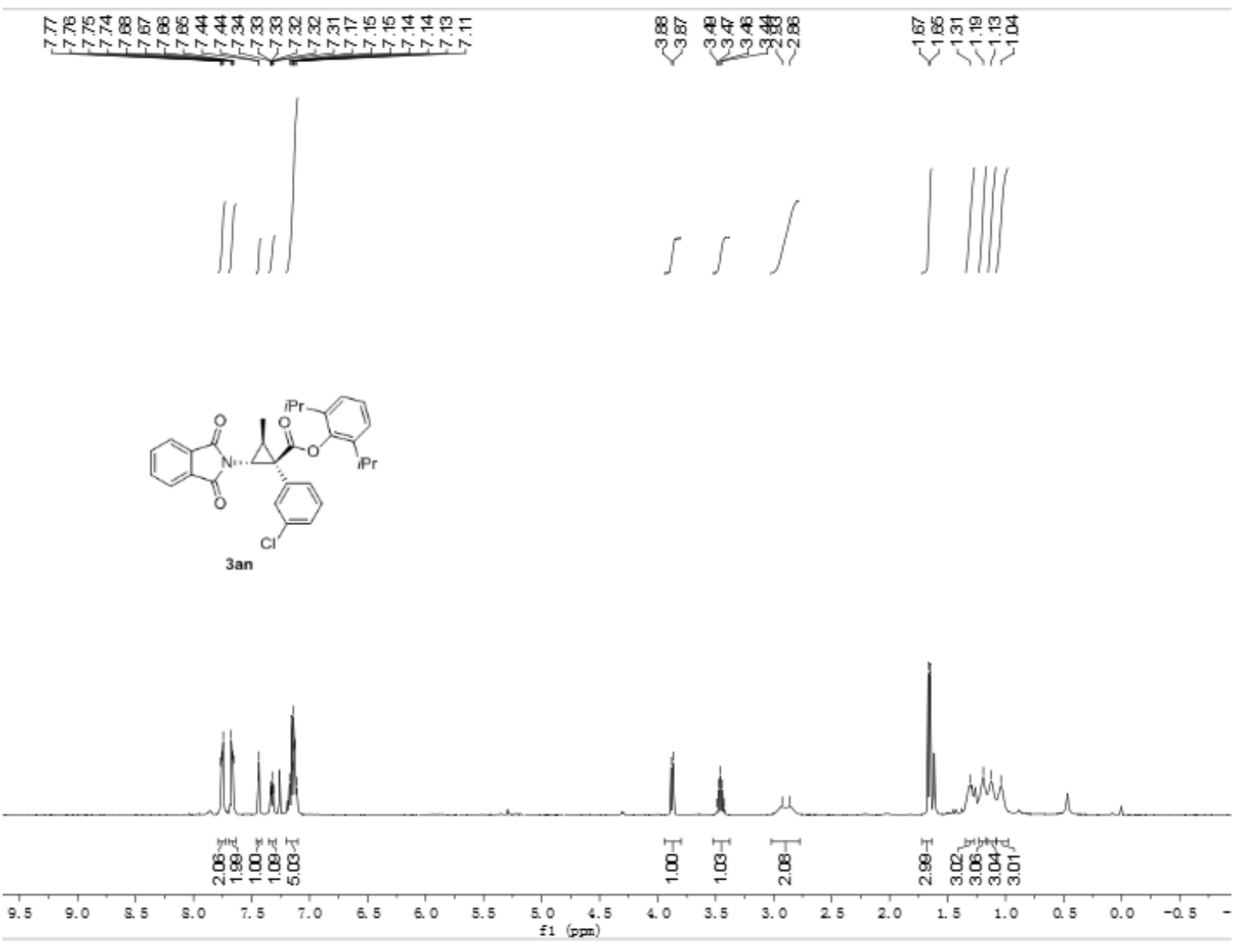

Figure S28: ${ }^{1} \mathrm{H} \mathrm{NMR}\left(400 \mathrm{MHz}, \mathrm{CDCl}_{3}\right)$ spectrum for 3an 


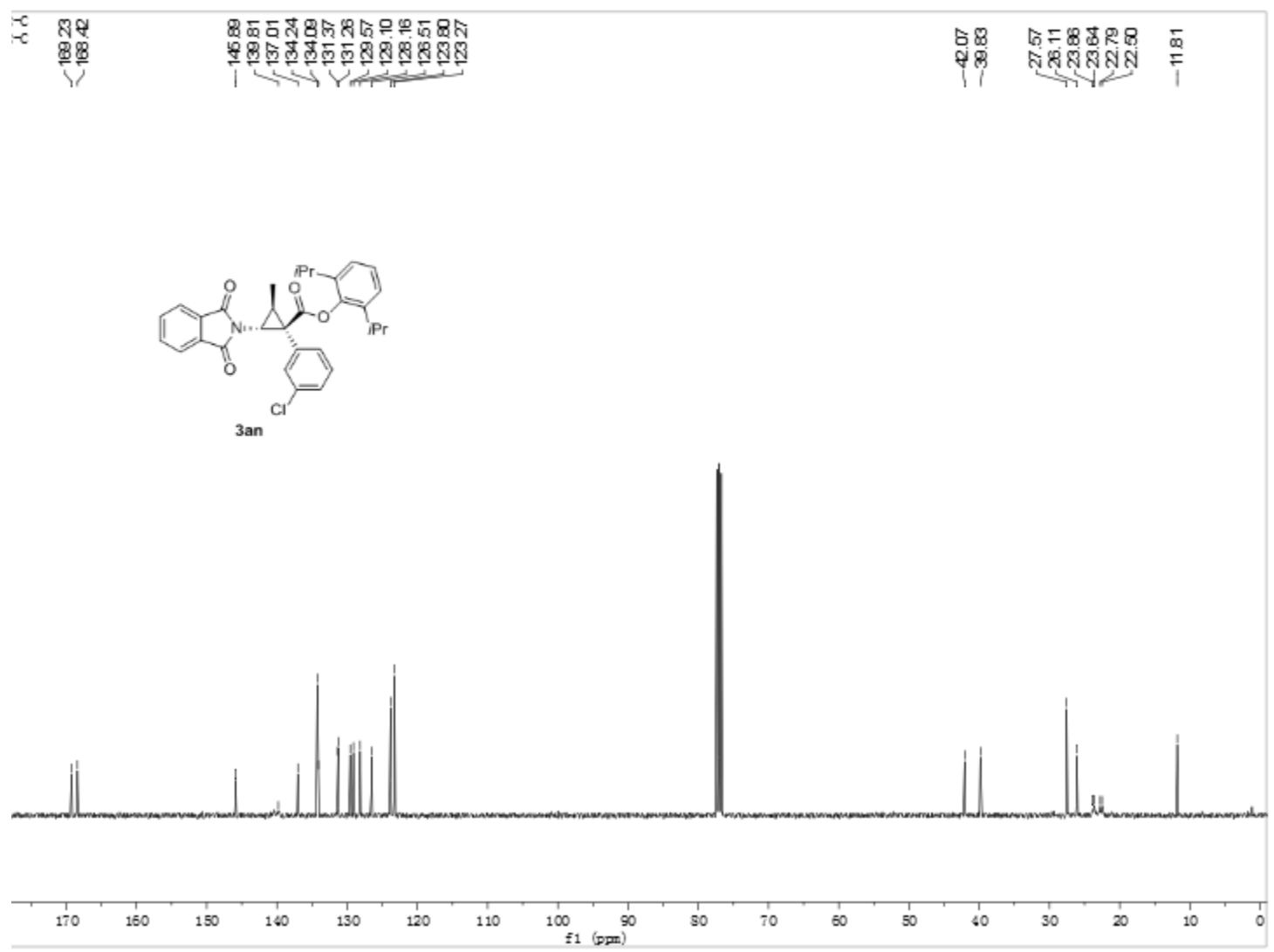

Figure S29: ${ }^{13} \mathrm{C}$ NMR $\left(100 \mathrm{MHz}, \mathrm{CDCl}_{3}\right)$ spectrum for 3an 


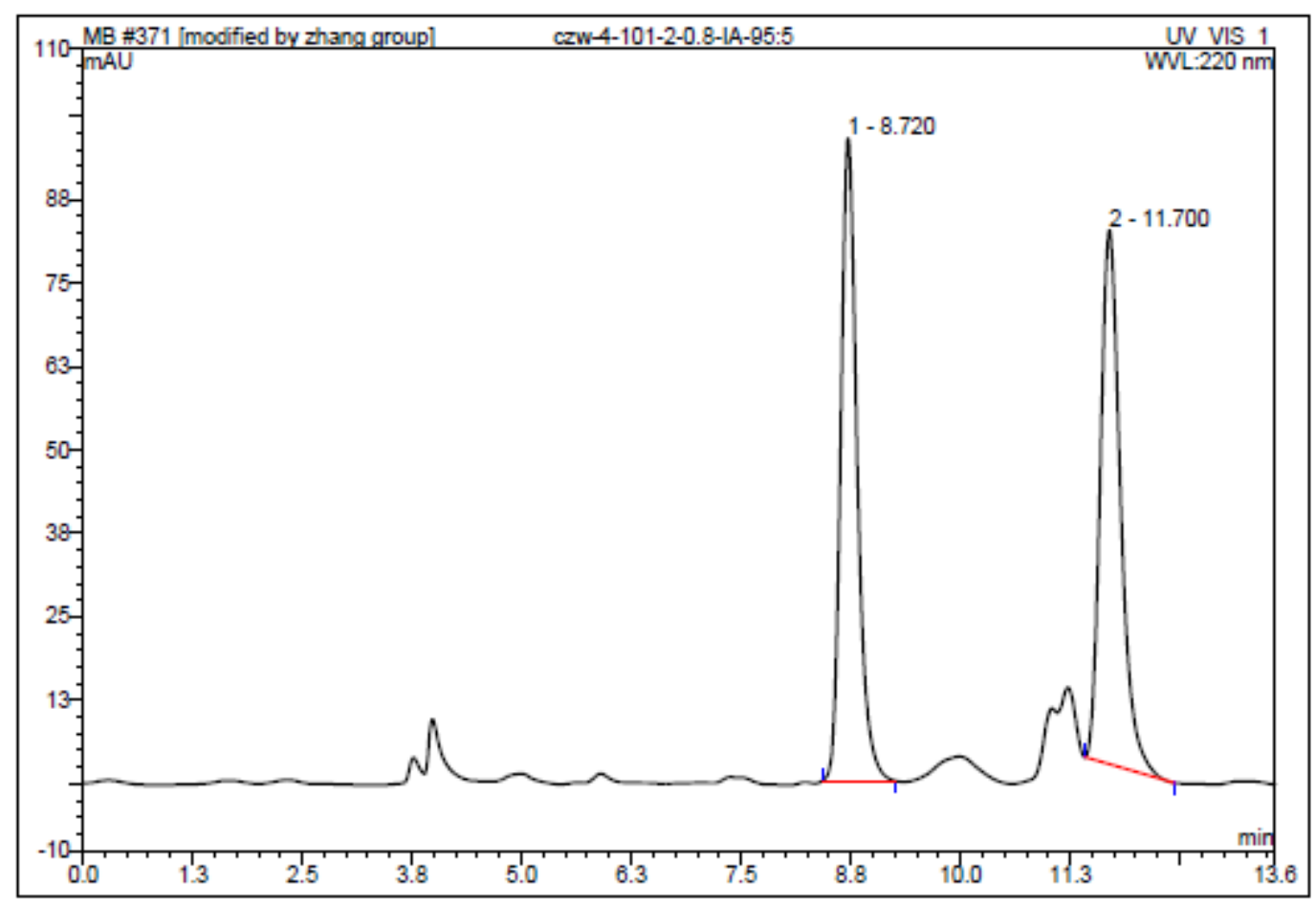

\begin{tabular}{|r|rrrrrrr|}
\hline No. & $\begin{array}{c}\text { Ret.Time } \\
\text { min }\end{array}$ & Peak Name & $\begin{array}{c}\text { Height } \\
\text { mAU }\end{array}$ & $\begin{array}{c}\text { Area } \\
\text { mAU*min }\end{array}$ & $\begin{array}{c}\text { Rel.Area } \\
\%\end{array}$ & Amount & Type \\
\hline 1 & 8.72 & n.a. & 96.210 & 20.893 & 49.54 & n.a. & BMB $^{\star}$ \\
2 & 11.70 & n.a. & 79.959 & 21.284 & 50.46 & n.a. & BMB $^{\star}$ \\
\hline Total: & & & 176.168 & 42.177 & 100.00 & 0.000 & \\
\hline
\end{tabular}

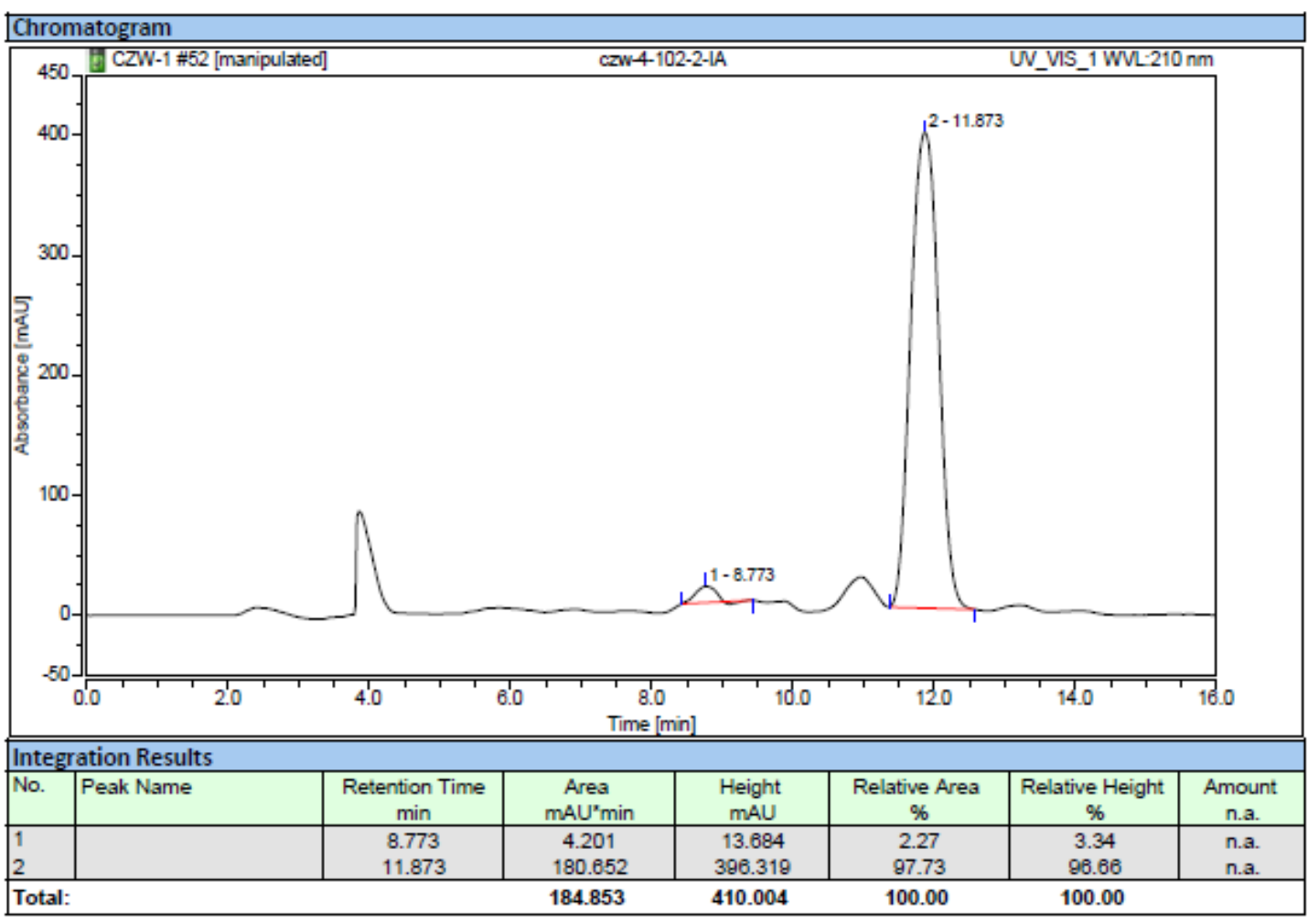

Figure S30: HPLC chromatographs of 3an 


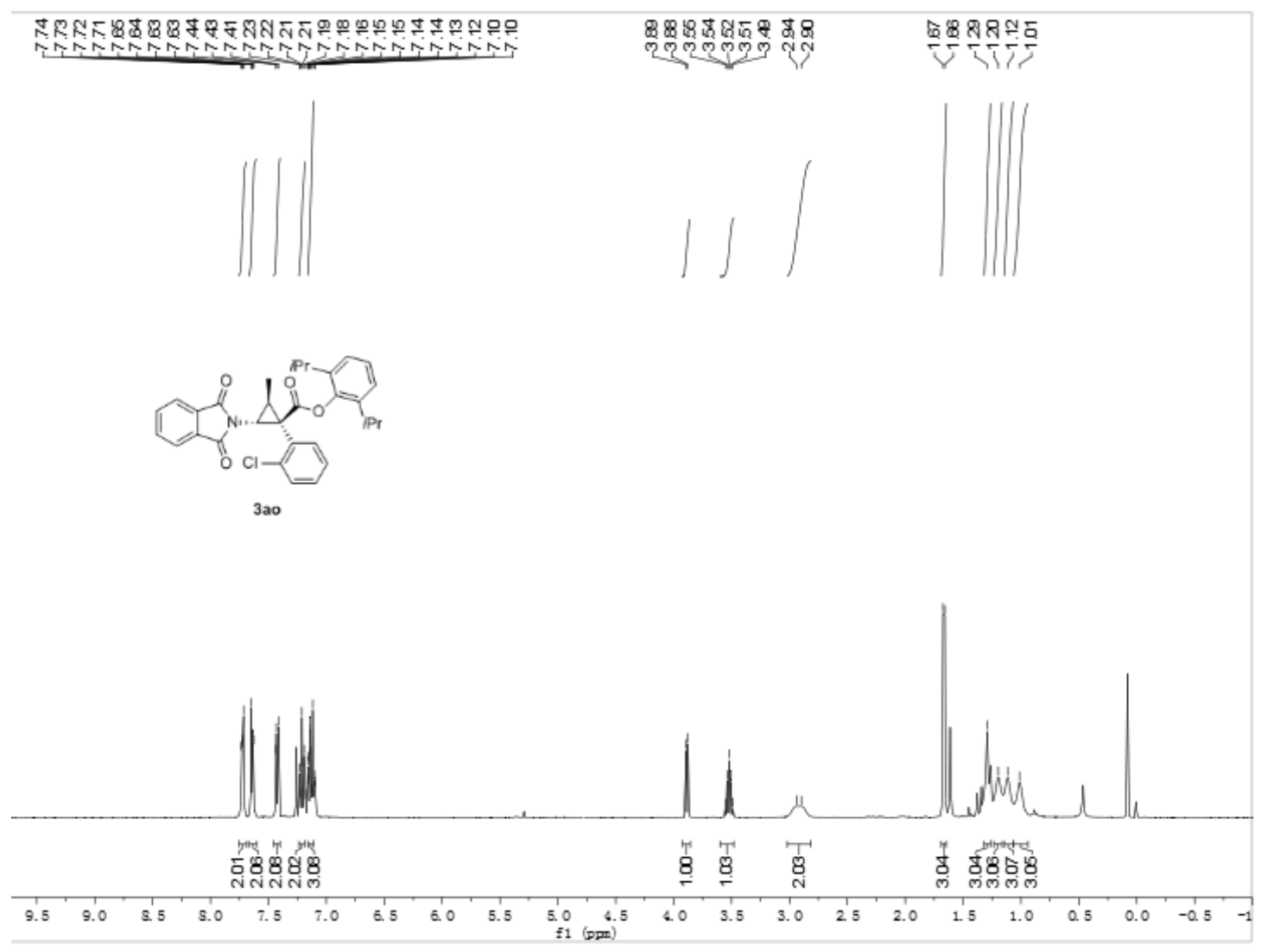

Figure S31: ${ }^{1} \mathrm{H}$ NMR $\left(400 \mathrm{MHz}, \mathrm{CDCl}_{3}\right)$ spectrum for $3 \mathbf{a o}$

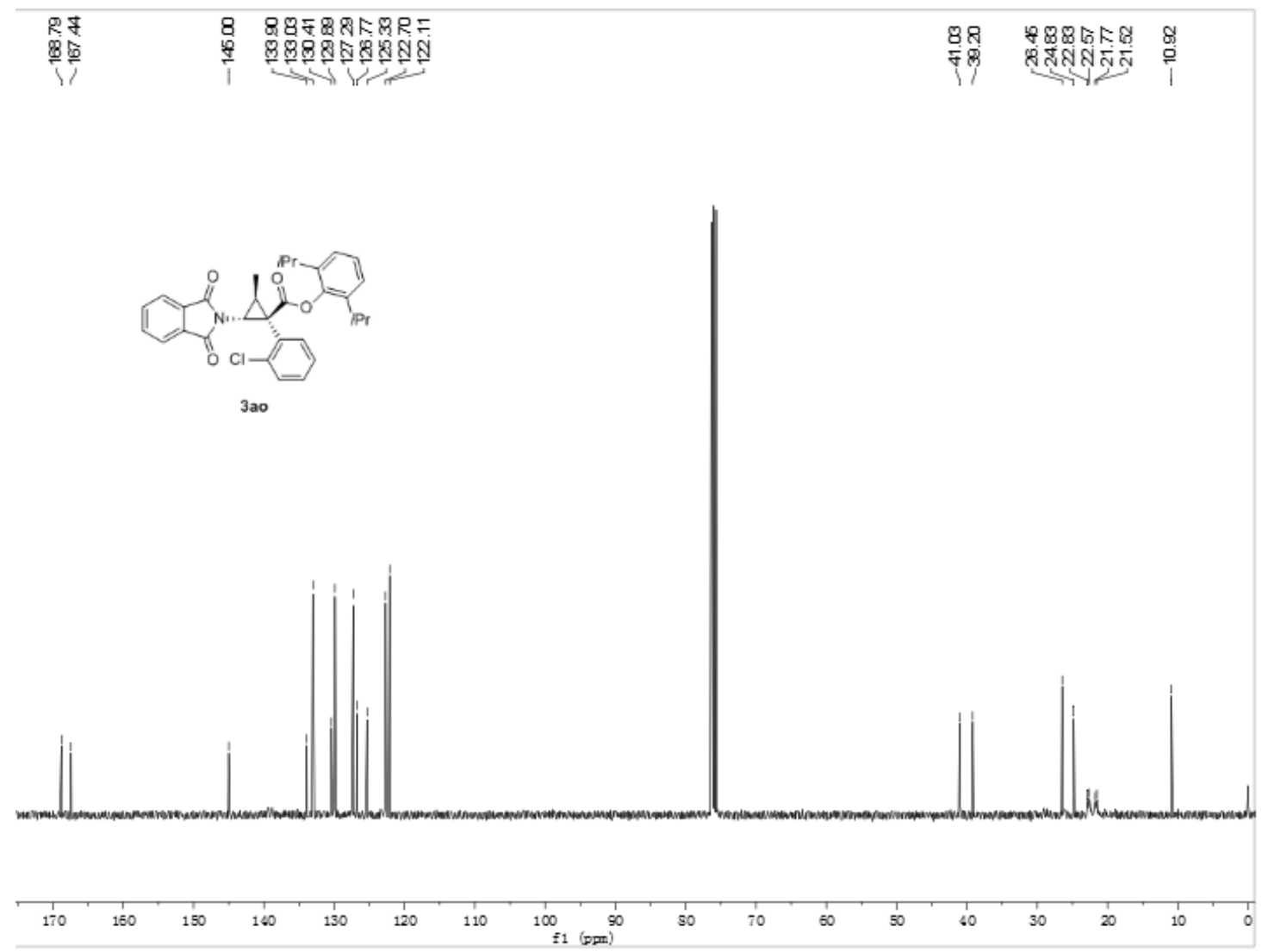


Figure S32: ${ }^{13} \mathrm{C}$ NMR $\left(100 \mathrm{MHz}, \mathrm{CDCl}_{3}\right)$ spectrum for 3ao
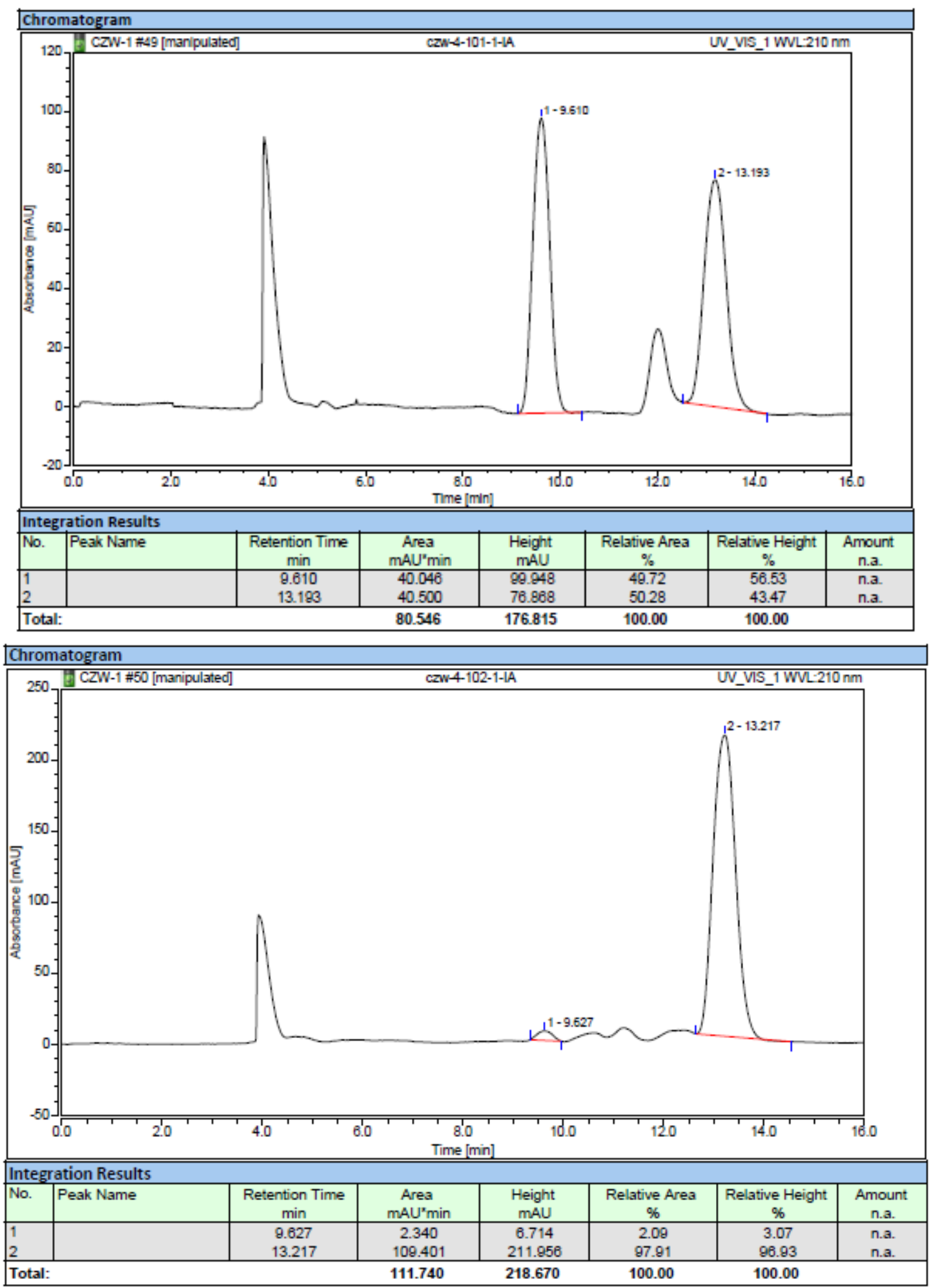

Figure S33: HPLC chromatographs of 3ao 


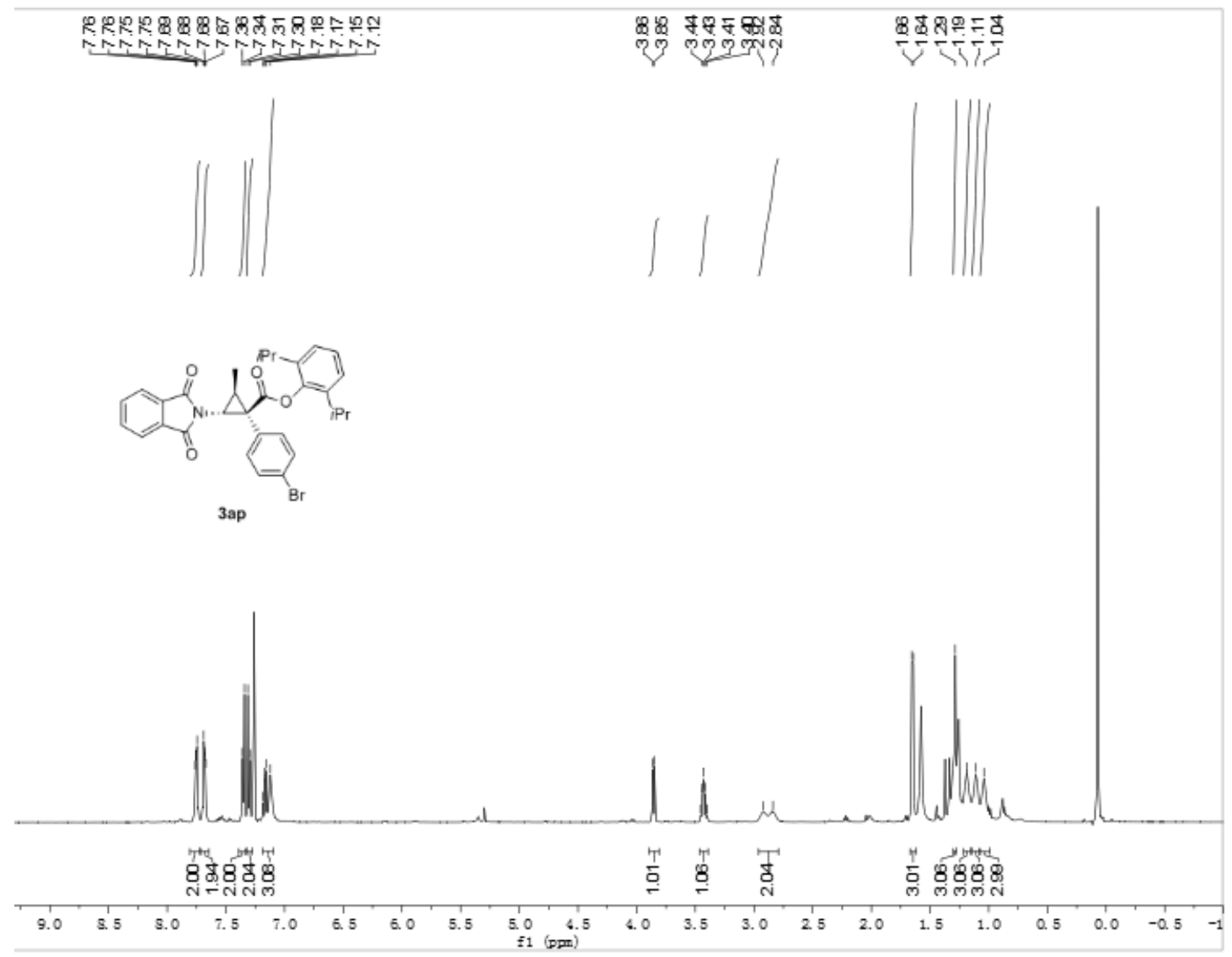

Figure S34: ${ }^{1} \mathrm{H} \mathrm{NMR}\left(500 \mathrm{MHz}, \mathrm{CDCl}_{3}\right)$ spectrum for 3ap 


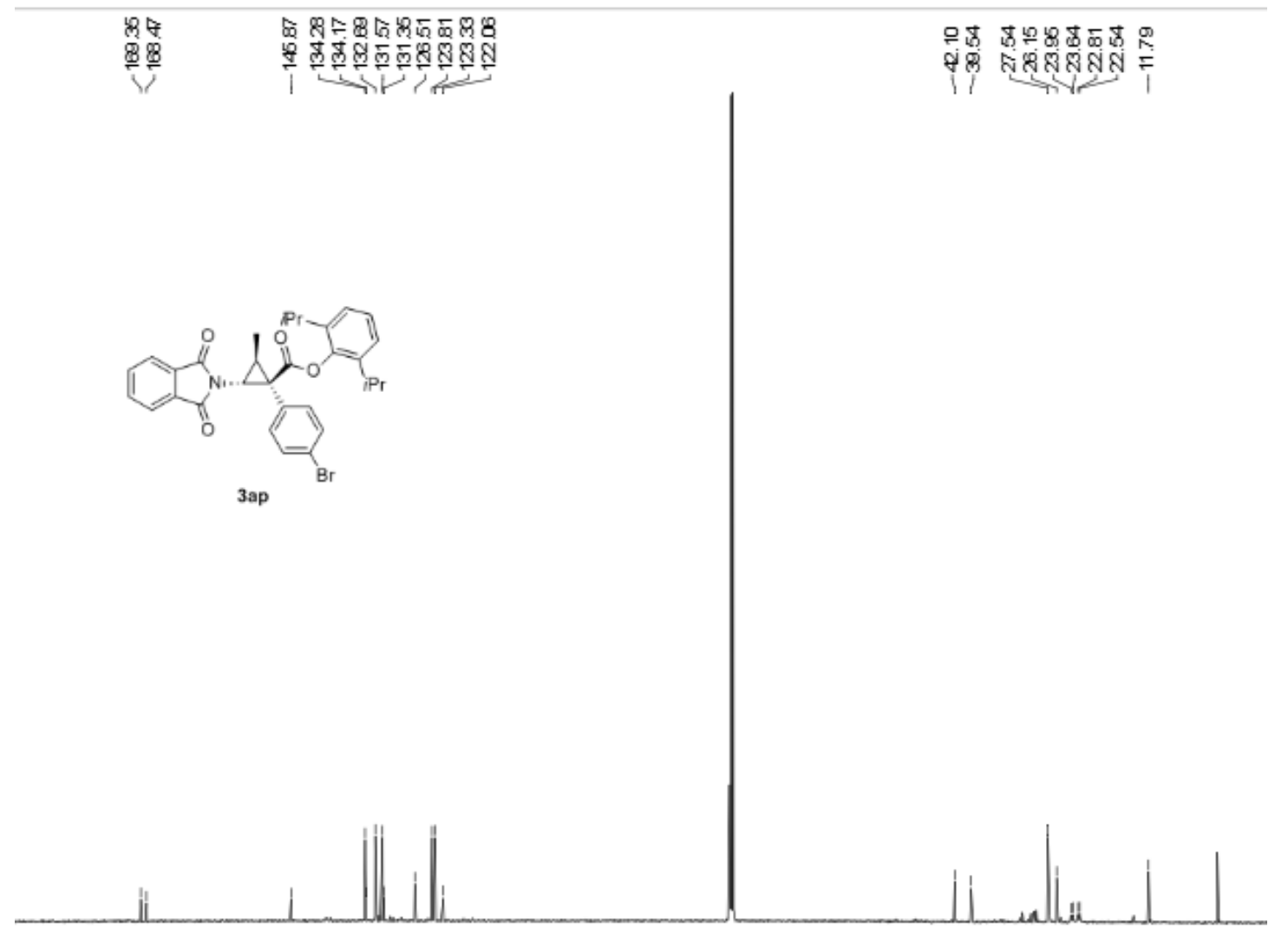

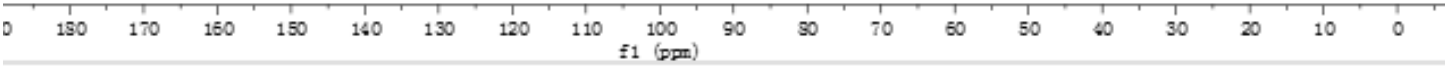

Figure S35: ${ }^{13} \mathrm{C} \mathrm{NMR}\left(125 \mathrm{MHz}, \mathrm{CDCl}_{3}\right)$ spectrum for 3ap 


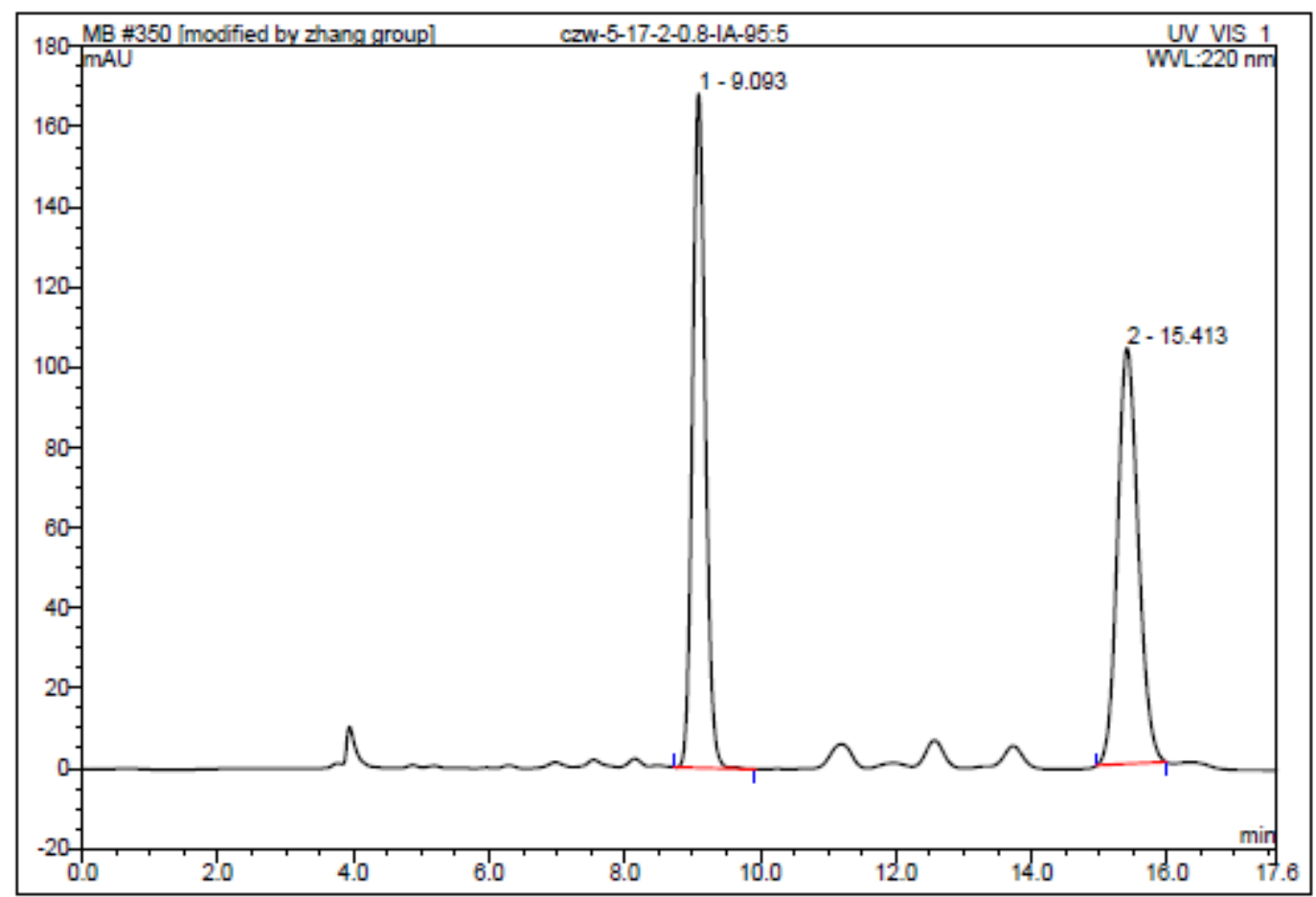

\begin{tabular}{|r|rrrrrrr|}
\hline No. & $\begin{array}{r}\text { Ret.Time } \\
\text { min }\end{array}$ & Peak Name & $\begin{array}{c}\text { Height } \\
\text { mAU }\end{array}$ & $\begin{array}{c}\text { Area } \\
\text { mAU*min }\end{array}$ & $\begin{array}{c}\text { Rel.Area } \\
\%\end{array}$ & Amount & Type \\
\hline 1 & 9.09 & n.a. & 168.166 & 36.825 & 49.96 & n.a. & BMB $^{\star}$ \\
2 & 15.41 & n.a. & 103.694 & 36.879 & 50.04 & n.a. & BMB $^{\star}$ \\
\hline Total: & & & 271.860 & 73.703 & 100.00 & 0.000 & \\
\hline
\end{tabular}

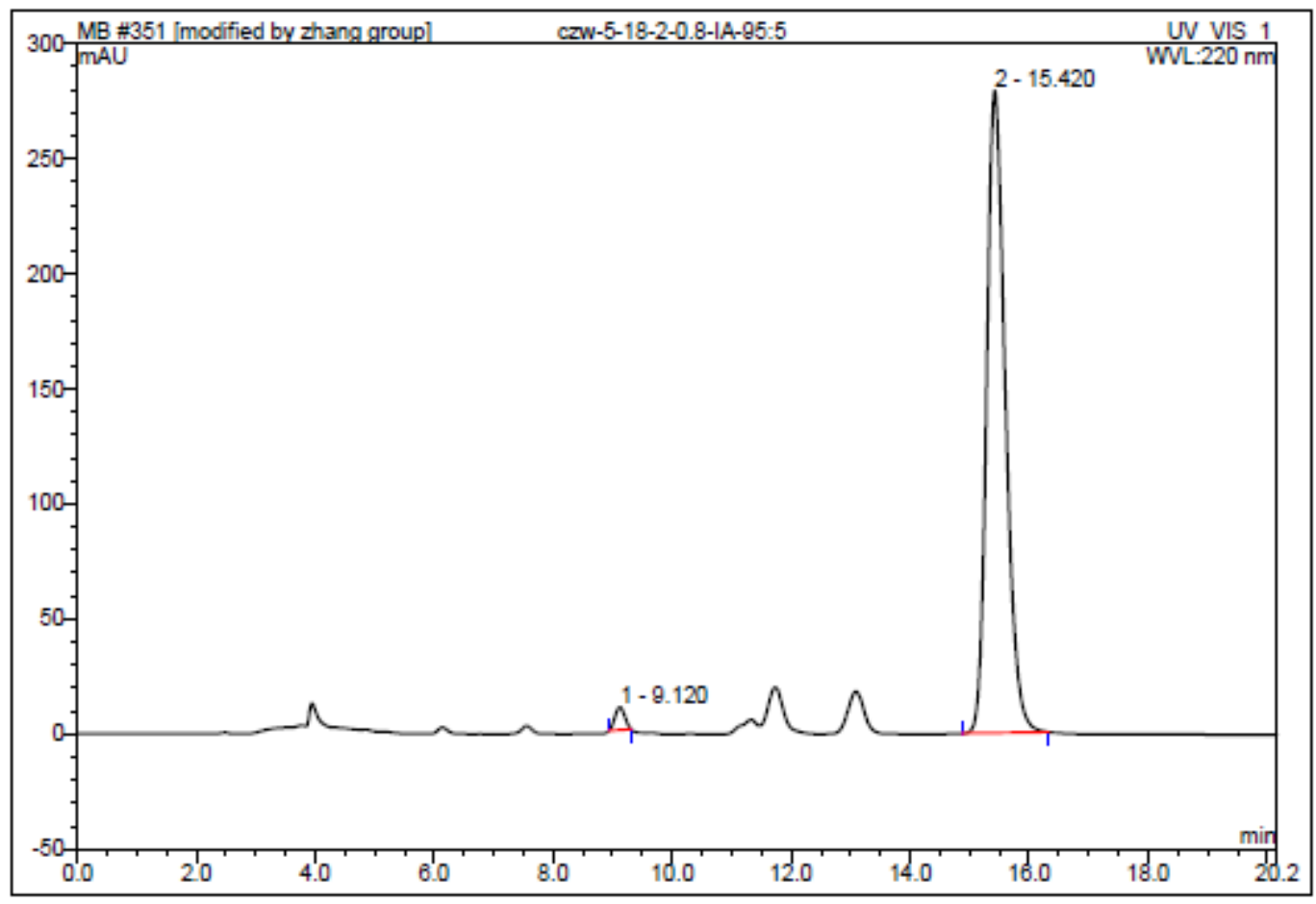

\begin{tabular}{|r|rrrrrrr|}
\hline No. & $\begin{array}{c}\text { Ret.Time } \\
\text { min }\end{array}$ & Peak Name & $\begin{array}{c}\text { Height } \\
\text { mAU }\end{array}$ & $\begin{array}{c}\text { Area } \\
\text { mAU*min }\end{array}$ & $\begin{array}{r}\text { Rel.Area } \\
\%\end{array}$ & Amount & Type \\
\hline 1 & 9.12 & n.a. & 9.802 & 1.873 & 1.81 & n.a. & BMB $^{\star}$ \\
2 & 15.42 & n.a. & 279.186 & 101.502 & 98.19 & n.a. & BMB $^{\star}$ \\
\hline Total: & & & 288.988 & 103.375 & 100.00 & 0.000 & \\
\hline
\end{tabular}


Figure S36: HPLC chromatographs of 3ap

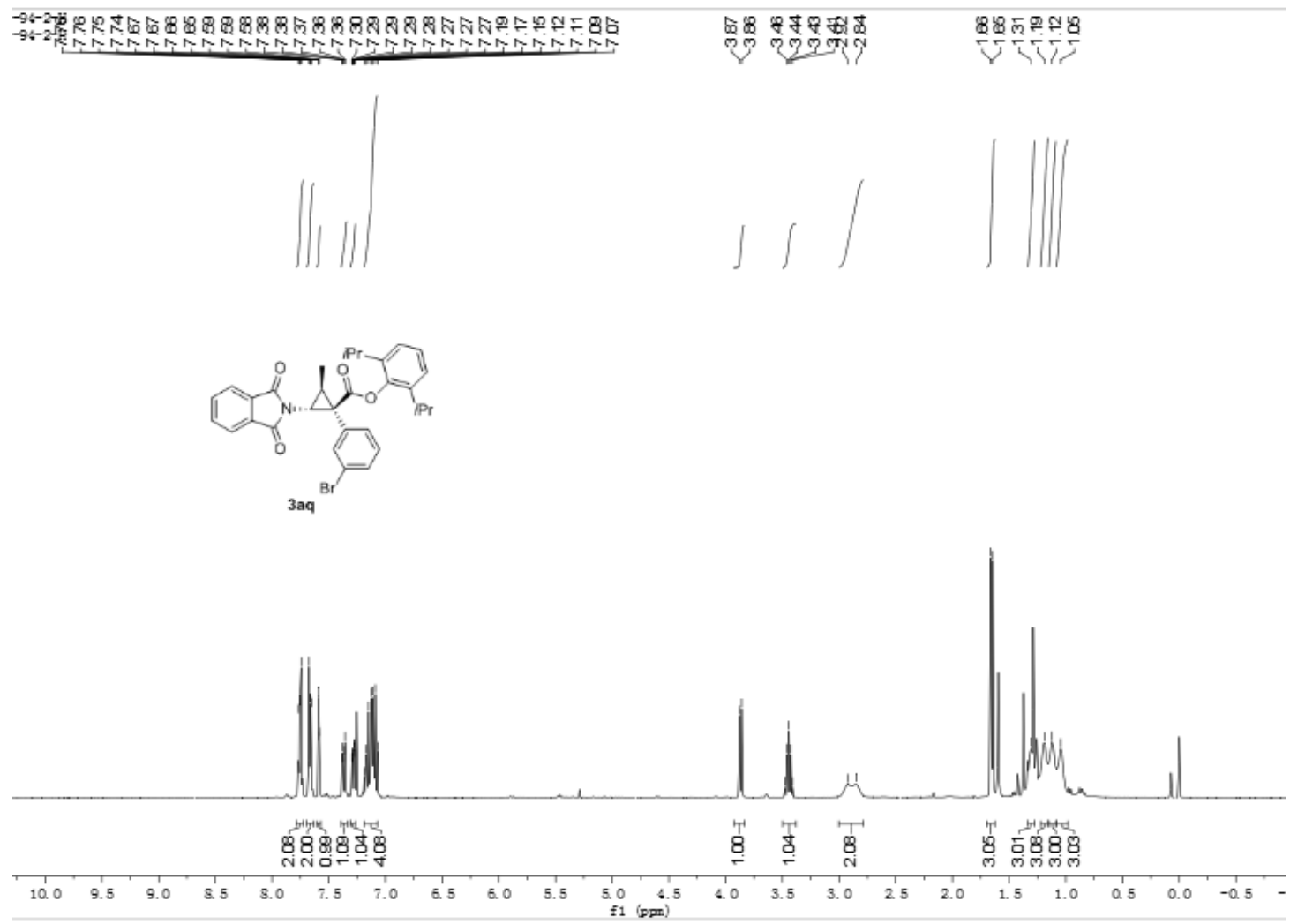

Figure S37: ${ }^{1} \mathrm{H} \mathrm{NMR}\left(400 \mathrm{MHz}, \mathrm{CDCl}_{3}\right)$ spectrum for 3aq

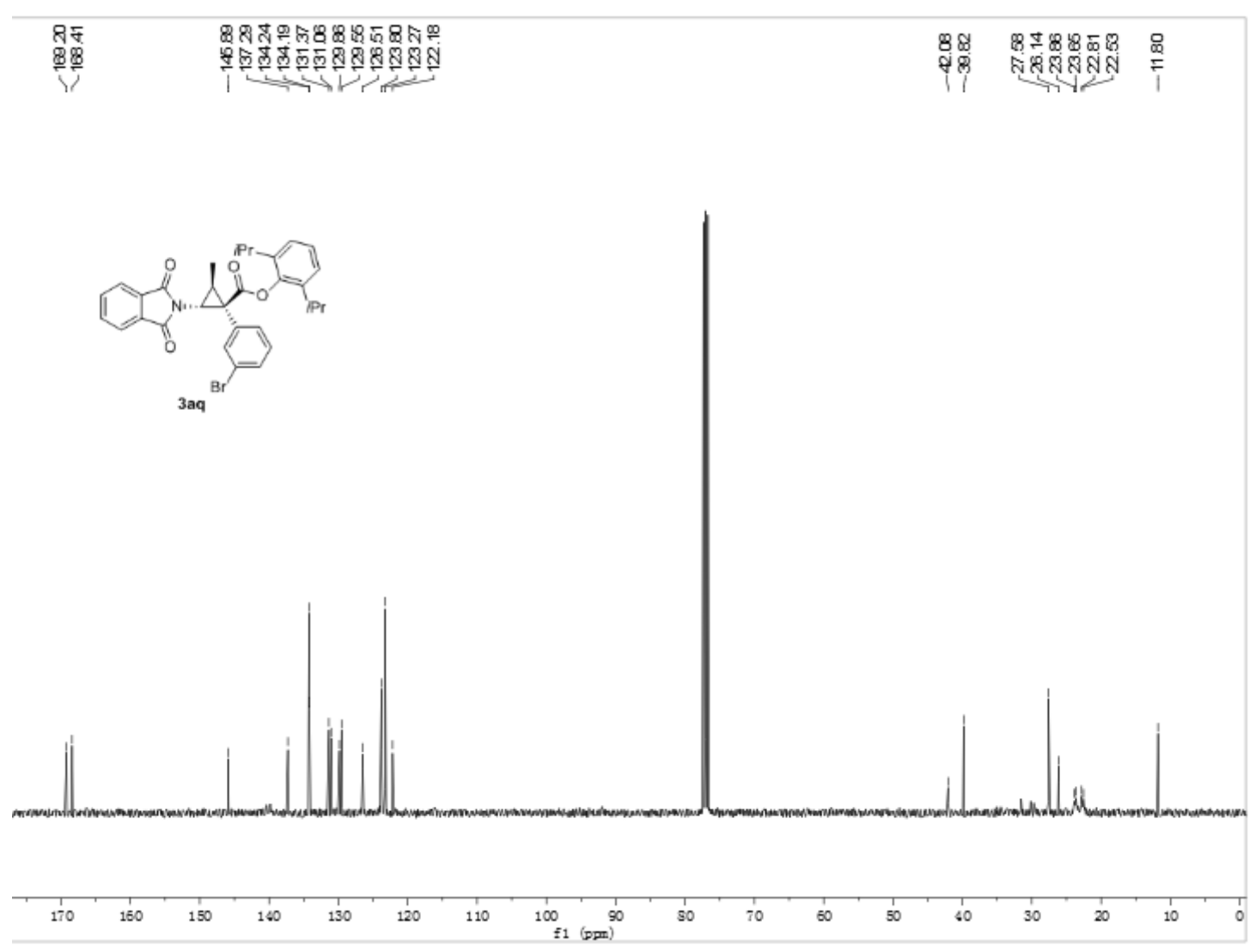

Figure S38: ${ }^{13} \mathrm{C}$ NMR $\left(100 \mathrm{MHz}, \mathrm{CDCl}_{3}\right)$ spectrum for 3aq 

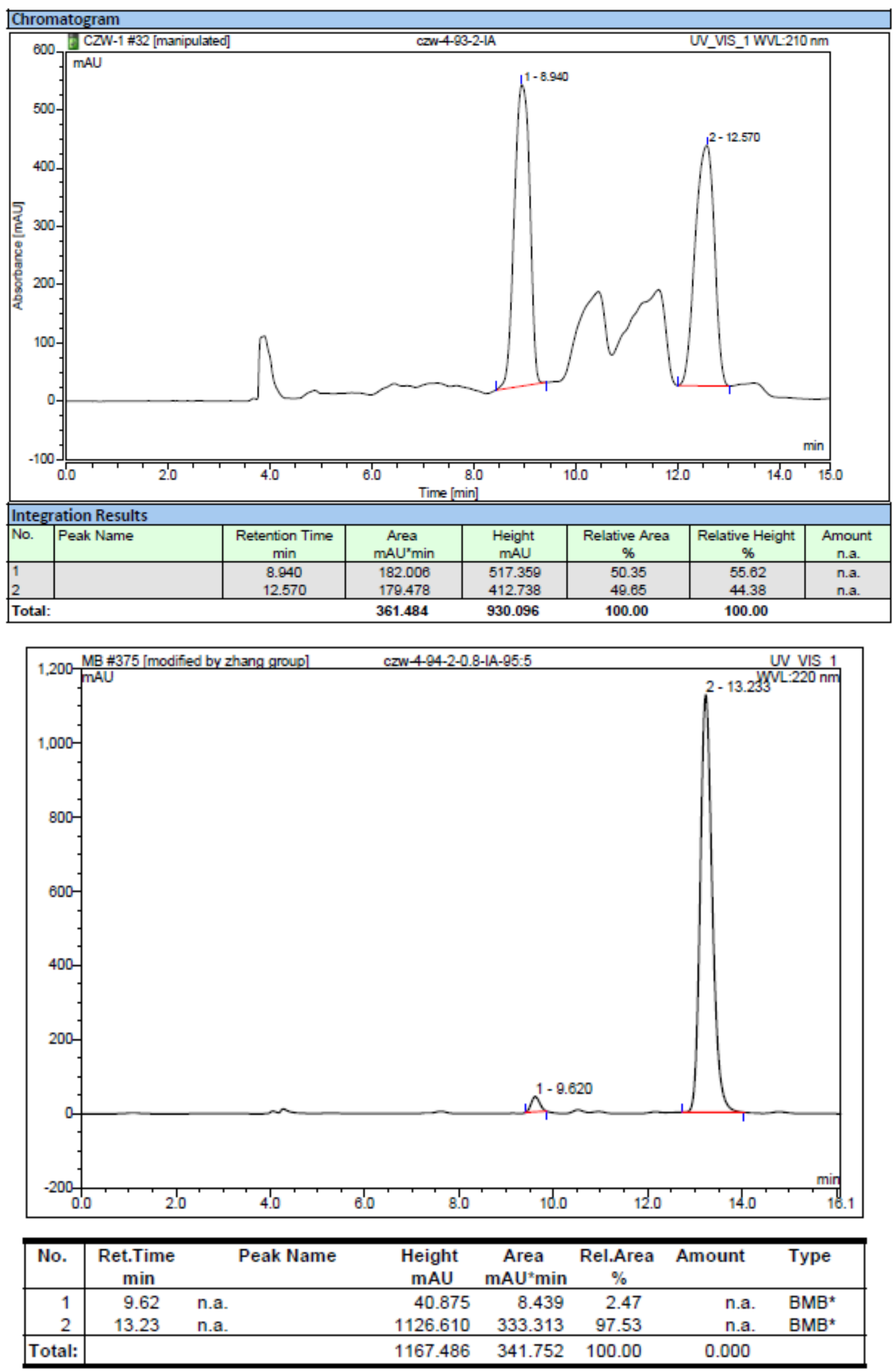

Figure S39: HPLC chromatographs of 3aq 


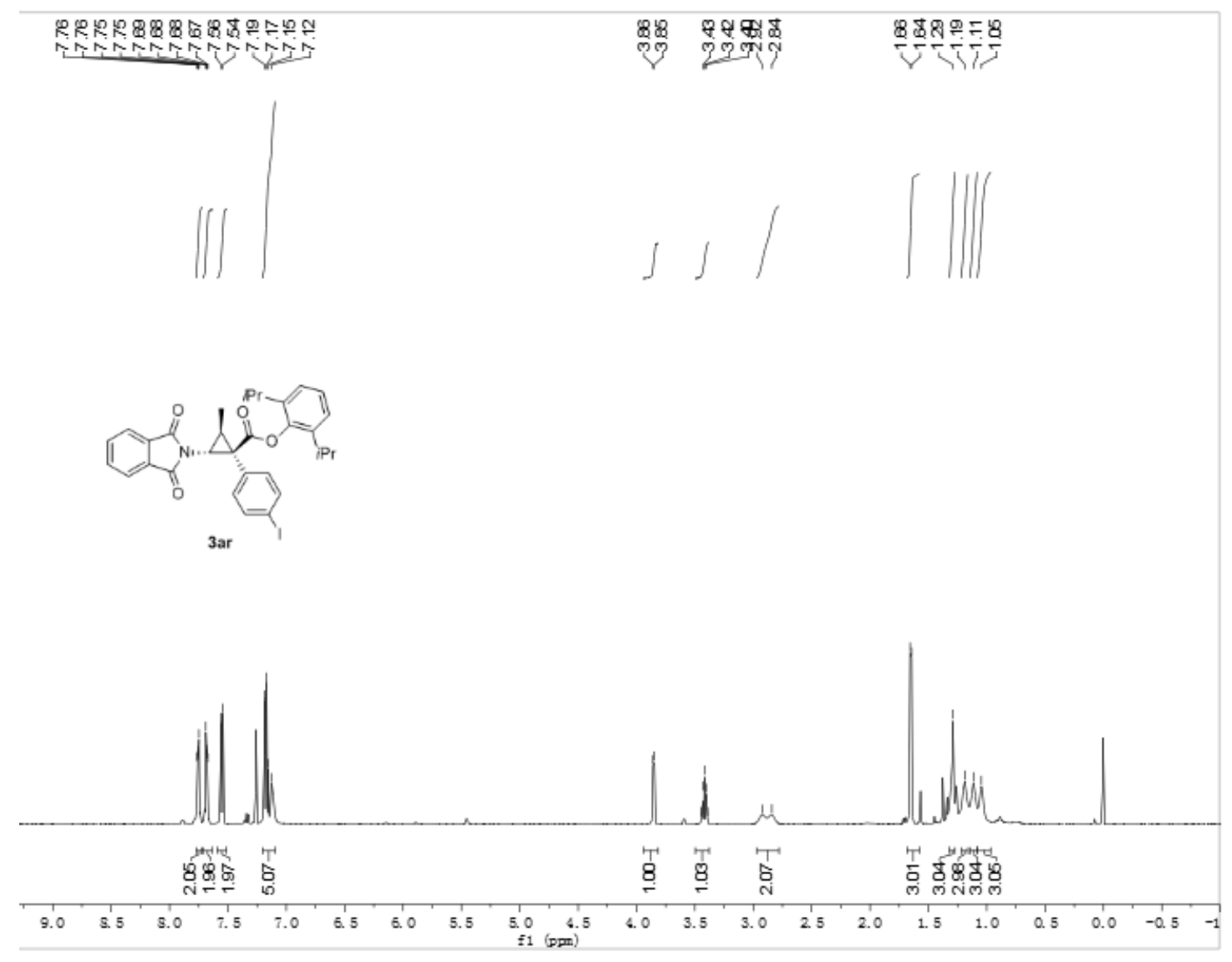

Figure S40: ${ }^{1} \mathrm{H}$ NMR $\left(500 \mathrm{MHz}, \mathrm{CDCl}_{3}\right)$ spectrum for $\mathbf{3 a r}$

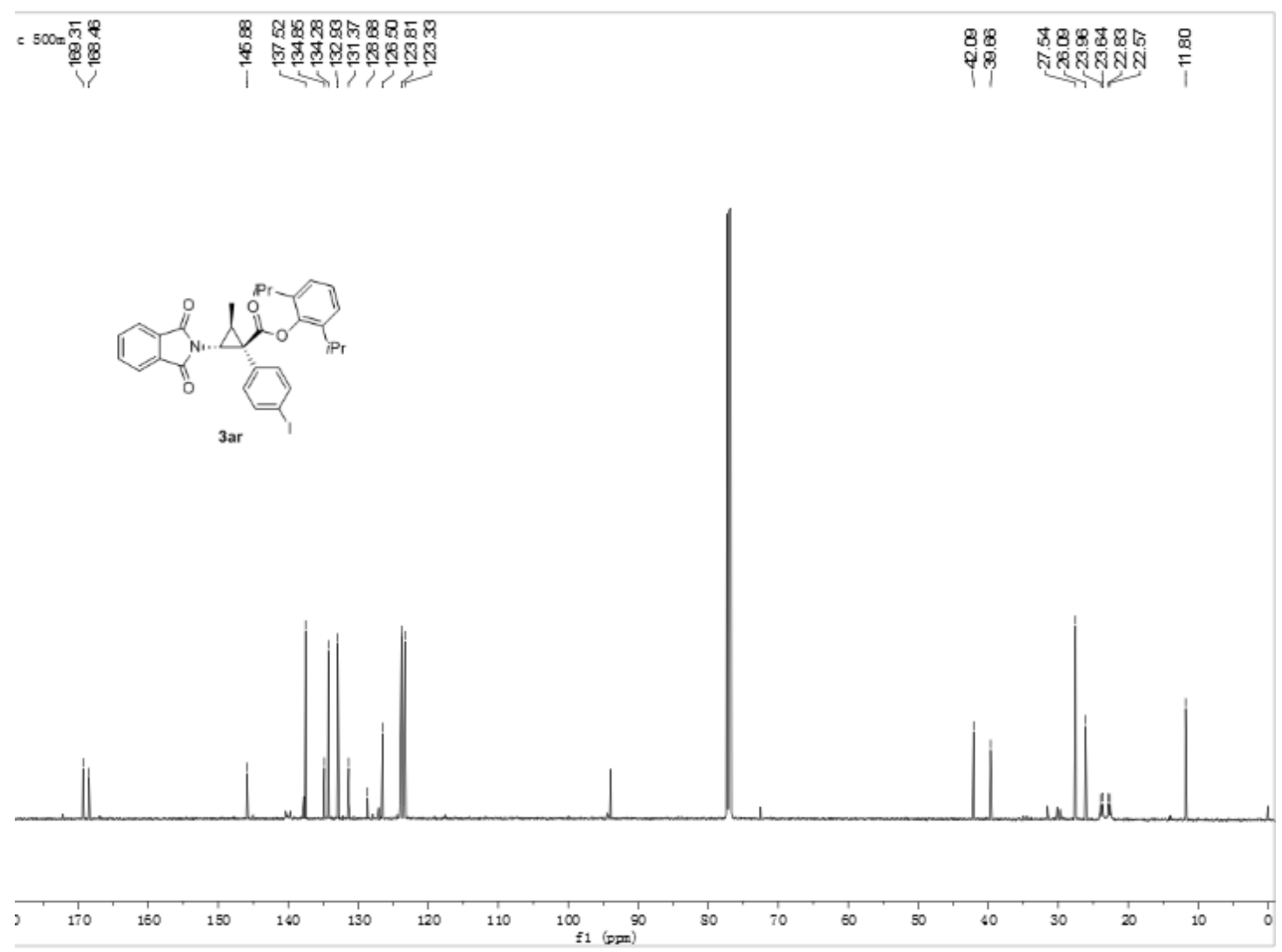


Figure S41: ${ }^{13} \mathrm{C}$ NMR (125 MHz, $\left.\mathrm{CDCl}_{3}\right)$ spectrum for 3ar

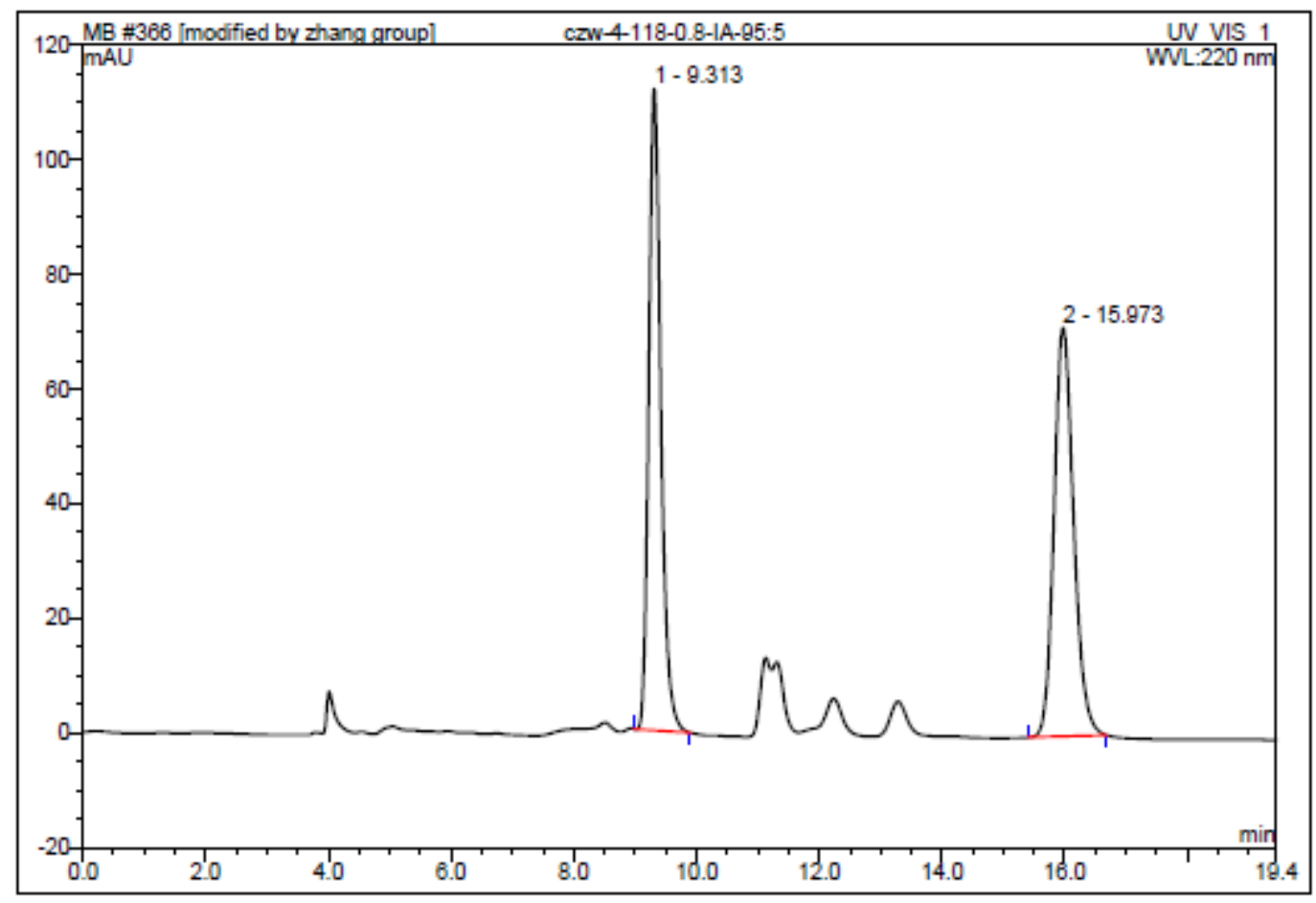

\begin{tabular}{|r|rrrrrrr|}
\hline No. & $\begin{array}{c}\text { Ret.Time } \\
\text { min }\end{array}$ & Peak Name & Height & Area & Rel.Area & Amount & Type \\
& & mAU & mAU*min & $\%$ & & \\
\hline 1 & 9.31 & n.a. & 111.886 & 25.588 & 49.24 & n.a. & BMB $^{\star}$ \\
2 & 15.97 & n.a. & 71.285 & 26.378 & 50.76 & n.a. & BMB $^{\star}$ \\
\hline Total: & & & 183.171 & 51.966 & 100.00 & 0.000 & \\
\hline
\end{tabular}

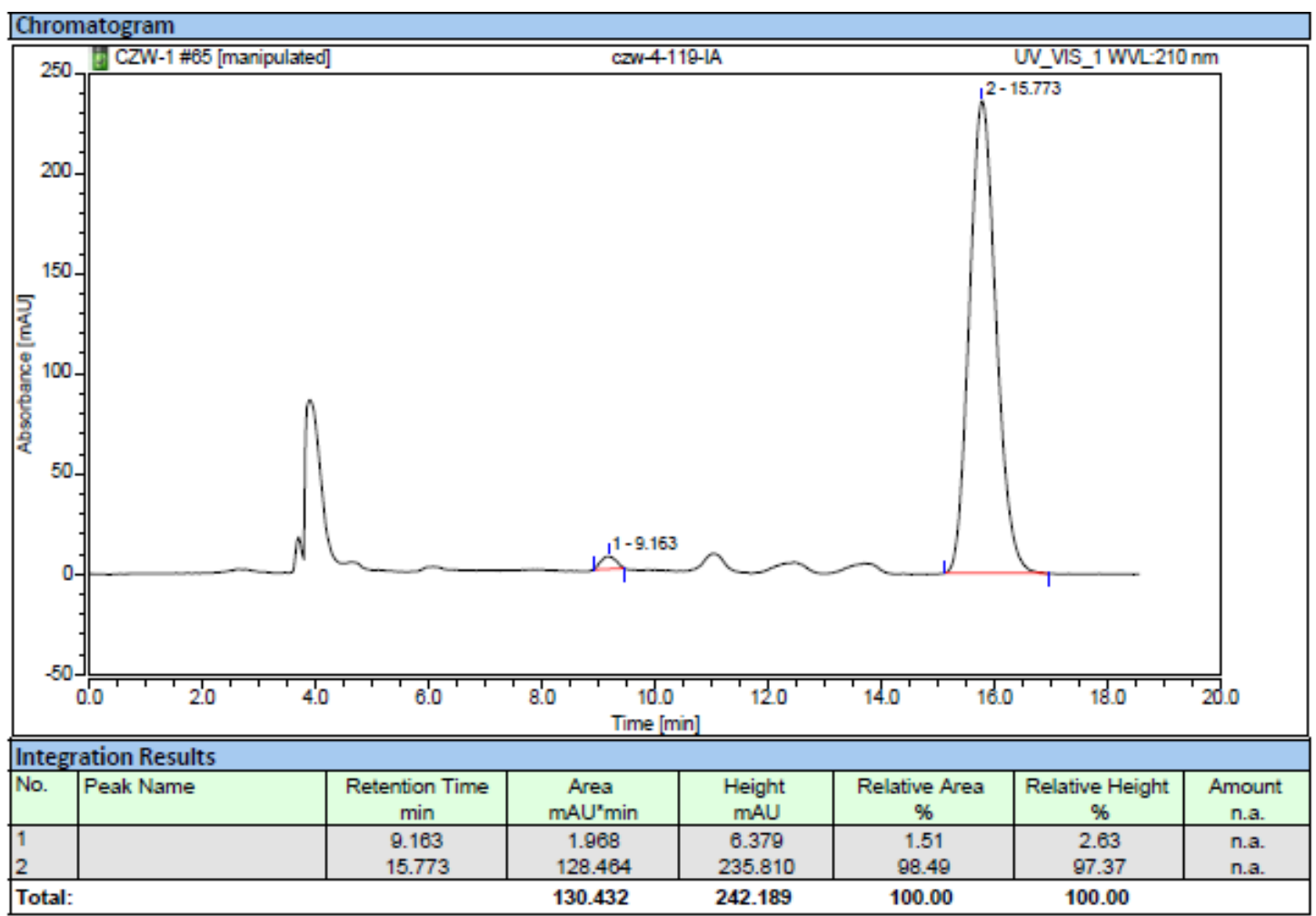

Figure S42: HPLC chromatographs of 3ar 


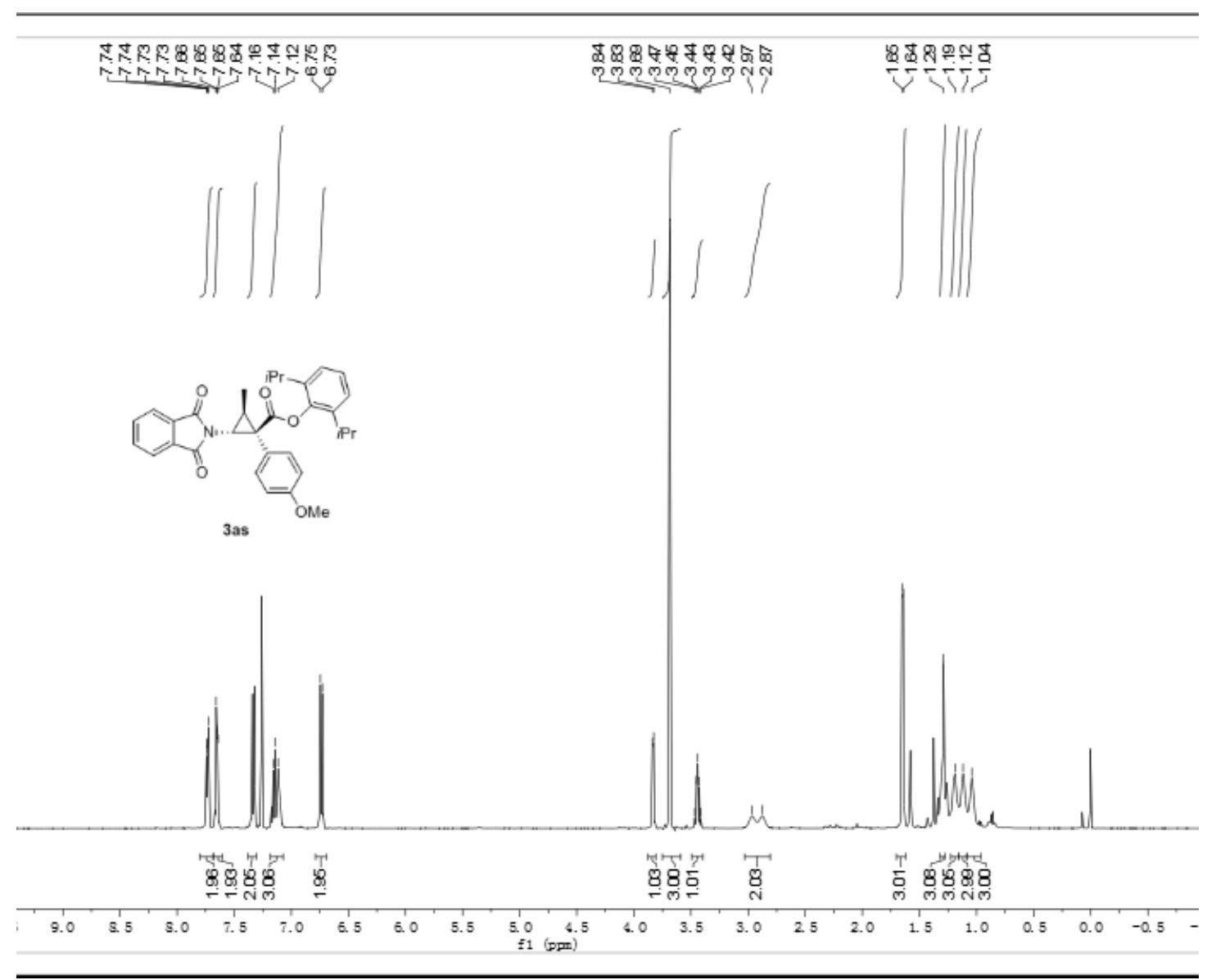

Figure S43: ${ }^{1} \mathrm{H}$ NMR $\left(500 \mathrm{MHz}, \mathrm{CDCl}_{3}\right)$ spectrum for 3as

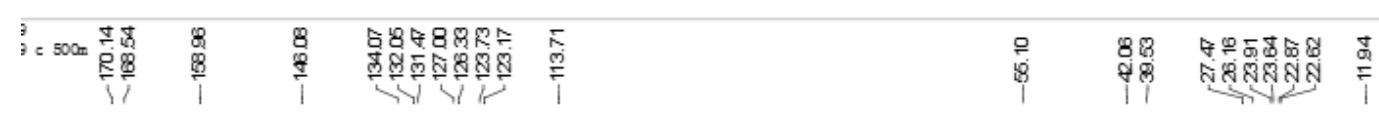

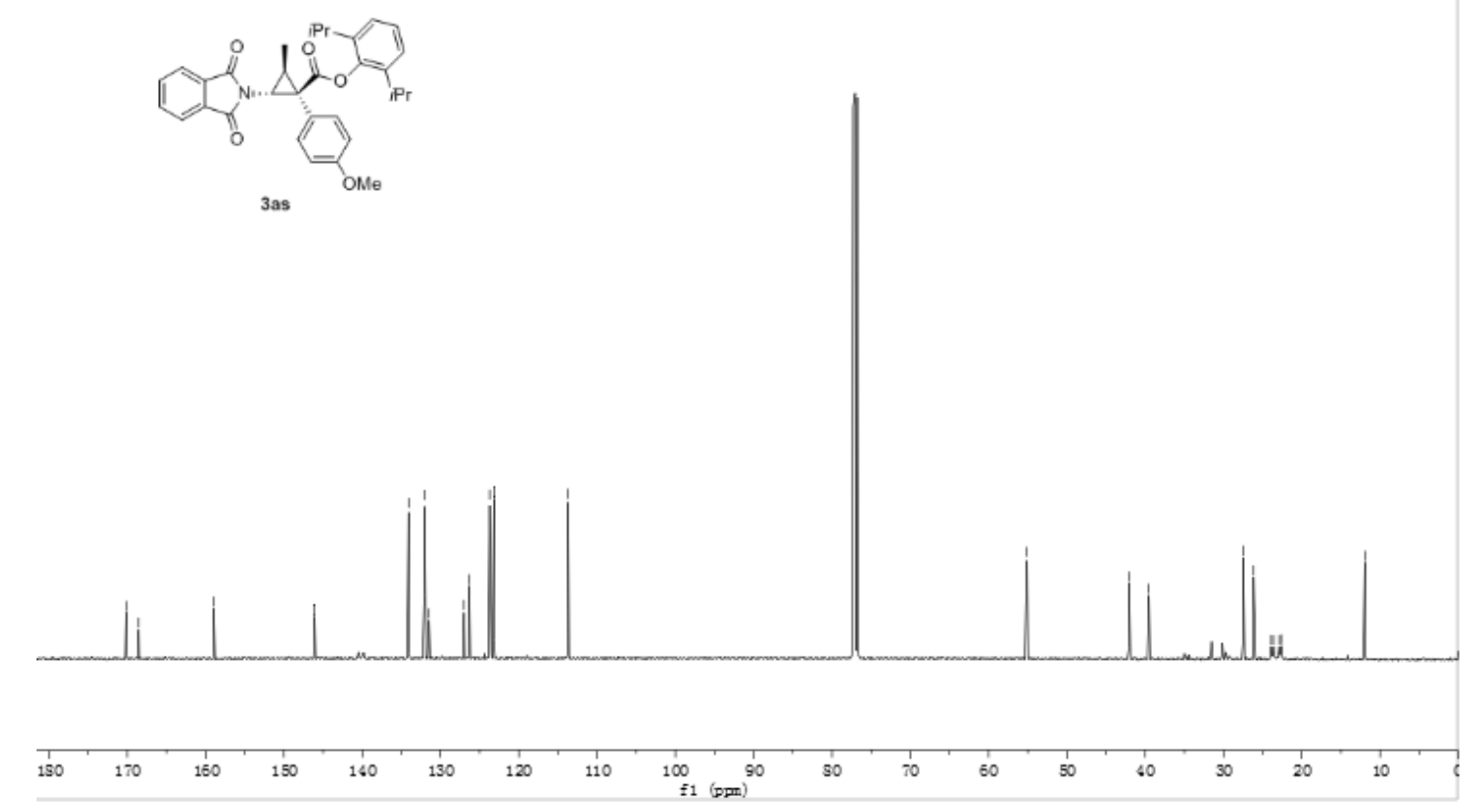

3as

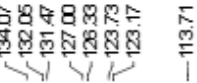

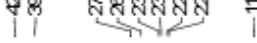

Figure S44: ${ }^{13} \mathrm{C}$ NMR (125 MHz, $\left.\mathrm{CDCl}_{3}\right)$ spectrum for 3as 


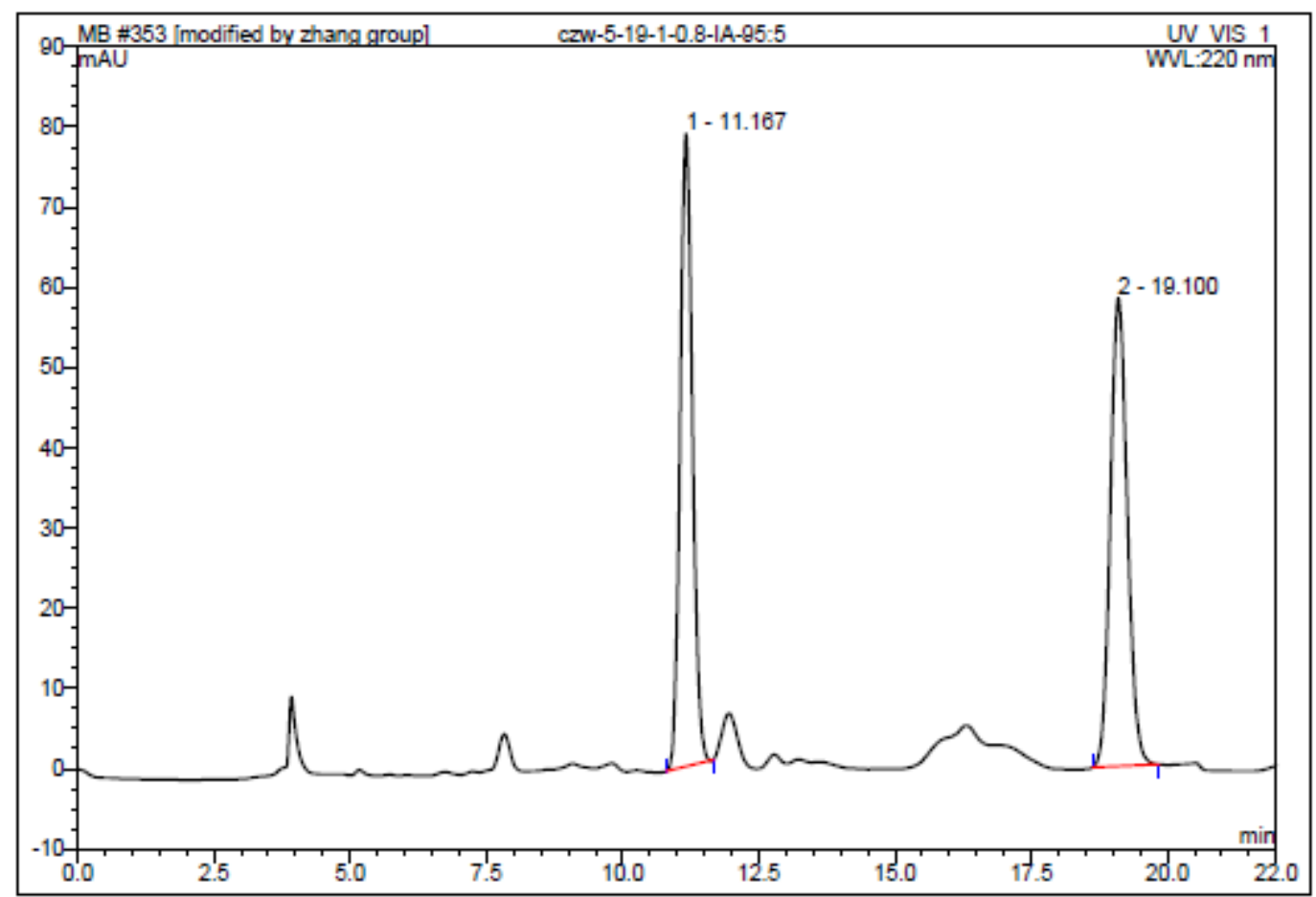

\begin{tabular}{|r|ccrrrrr|}
\hline No. & $\begin{array}{c}\text { Ret.Time } \\
\text { min }\end{array}$ & Peak Name & $\begin{array}{c}\text { Height } \\
\text { mAU }\end{array}$ & $\begin{array}{c}\text { Area } \\
\text { mAU*min }\end{array}$ & $\begin{array}{c}\text { Rel.Area } \\
\%\end{array}$ & Amount & Type \\
\hline 1 & 11.17 & n.a. & 78.886 & 20.782 & 49.56 & n.a. & BMB $^{\star}$ \\
2 & 19.10 & n.a. & 58.367 & 21.150 & 50.44 & n.a. & BMB $^{\star}$ \\
\hline Total: & & & 137.253 & 41.932 & 100.00 & 0.000 & \\
\hline
\end{tabular}

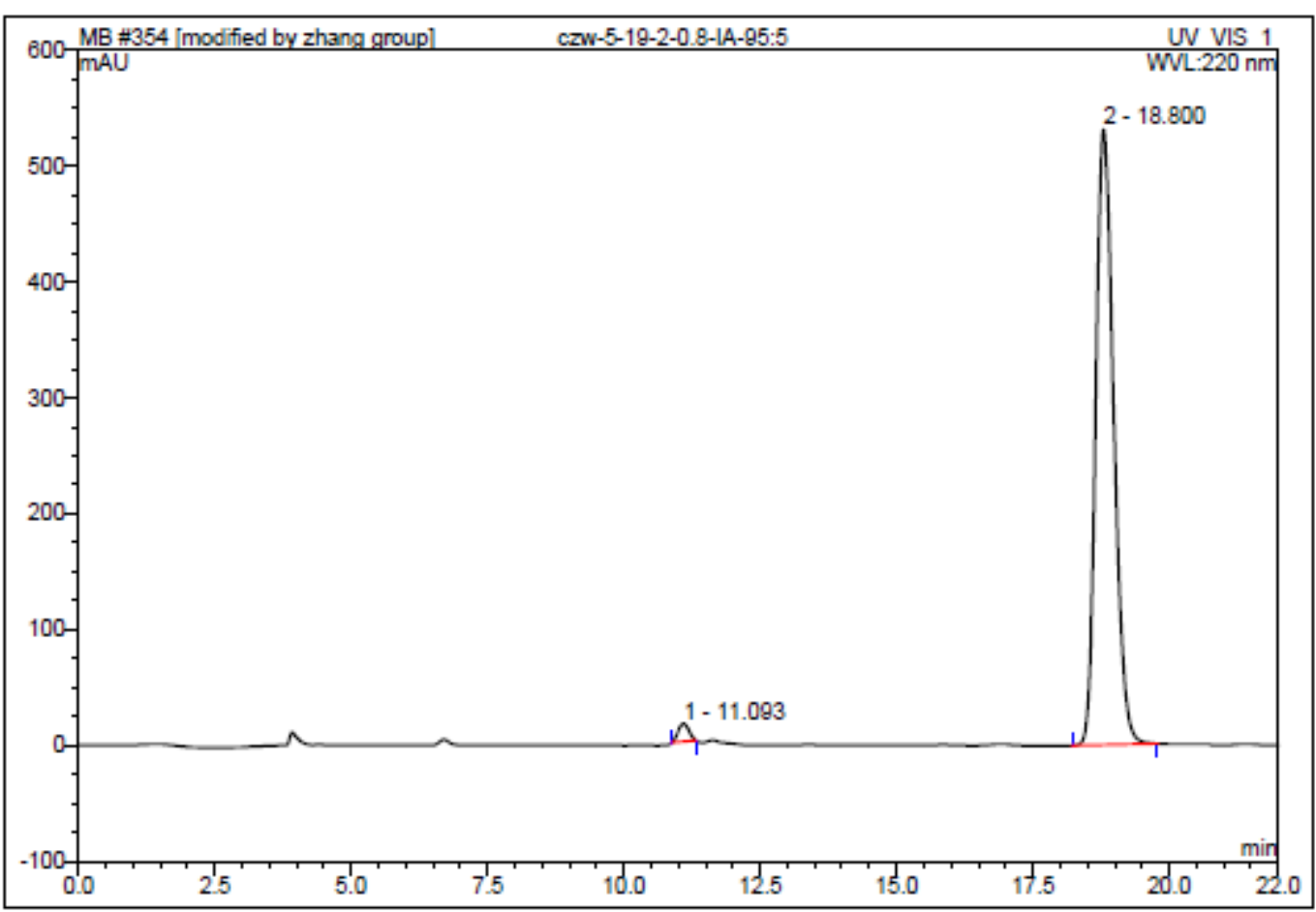

\begin{tabular}{|r|rrrrrrr|}
\hline No. & $\begin{array}{c}\text { Ret.Time } \\
\text { min }\end{array}$ & Peak Name & $\begin{array}{c}\text { Height } \\
\text { mAU }\end{array}$ & $\begin{array}{c}\text { Area } \\
\text { mAU*min }\end{array}$ & $\begin{array}{r}\text { Rel.Area } \\
\%\end{array}$ & Amount & Type \\
\hline 1 & 11.09 & n.a. & 15.507 & 3.535 & 1.72 & n.a. & BMB $^{\star}$ \\
2 & 18.80 & n.a. & 531.146 & 202.033 & 98.28 & n.a. & BMB $^{\star}$ \\
\hline Total: & & & 546.652 & 205.568 & 100.00 & 0.000 & \\
\hline
\end{tabular}


Figure S45: HPLC chromatographs of 3as

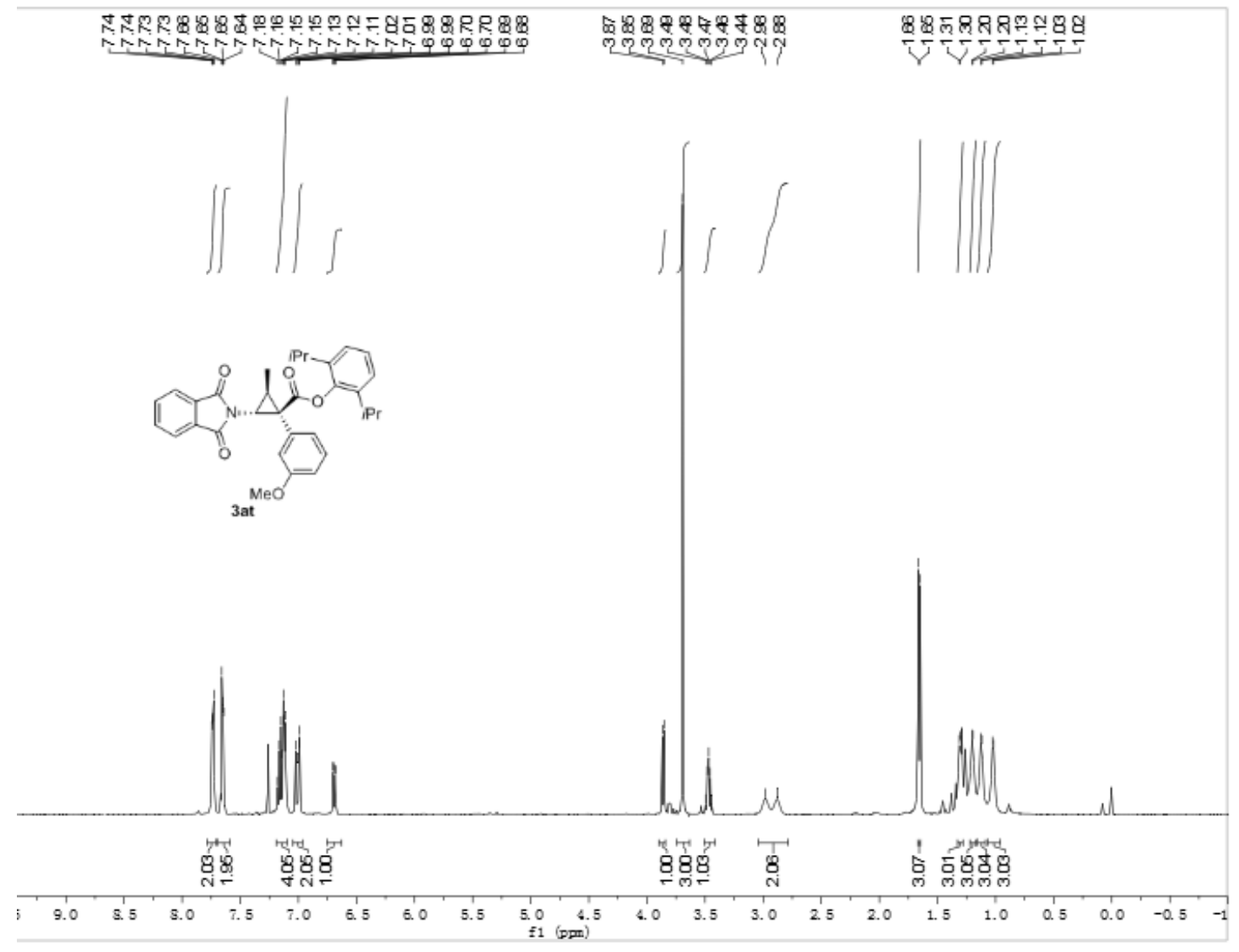

Figure S46: ${ }^{1} \mathrm{H}$ NMR $\left(500 \mathrm{MHz}, \mathrm{CDCl}_{3}\right)$ spectrum for 3at

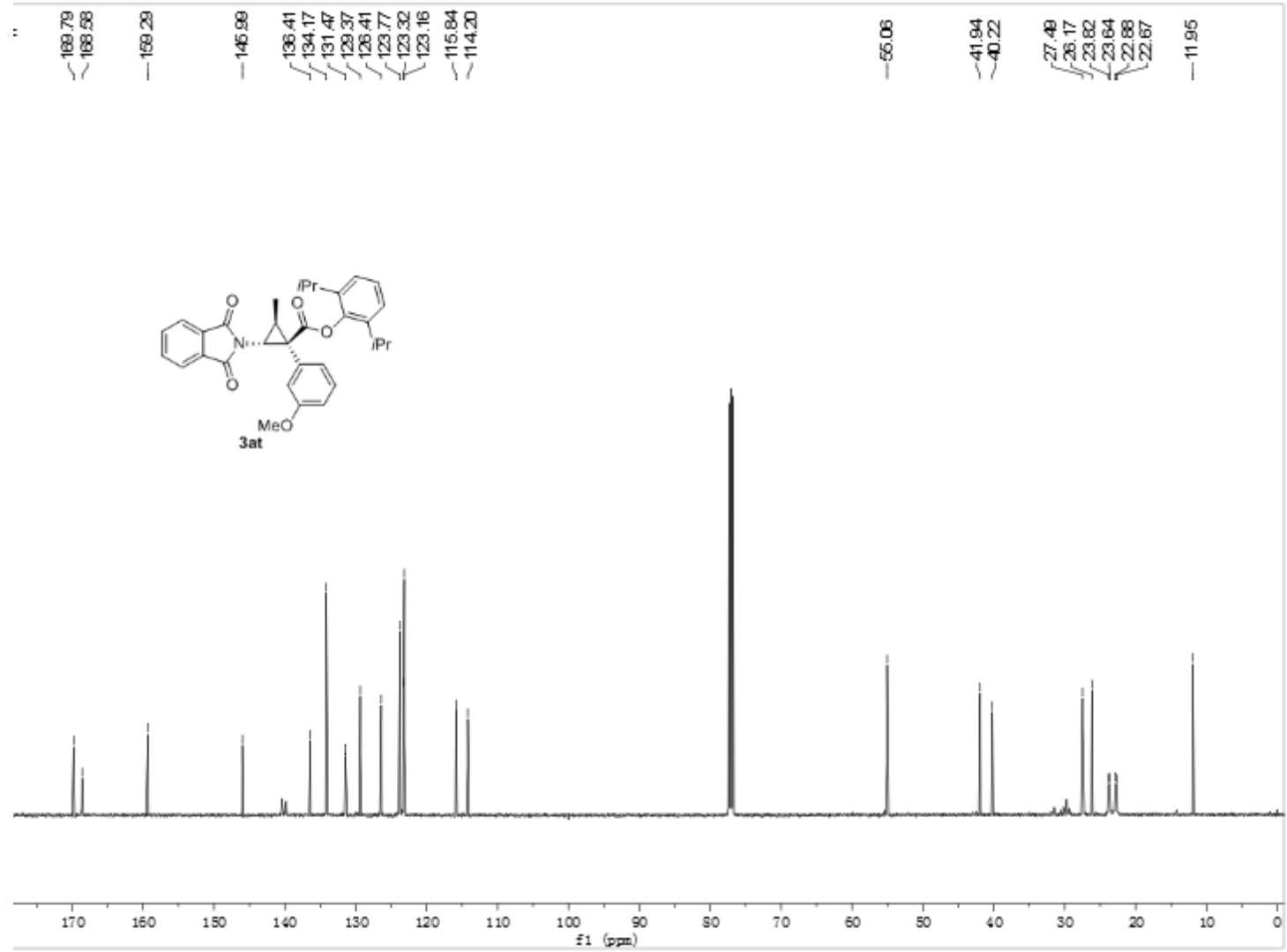


Figure S47: ${ }^{13} \mathrm{C}$ NMR (125 MHz, $\left.\mathrm{CDCl}_{3}\right)$ spectrum for 3at

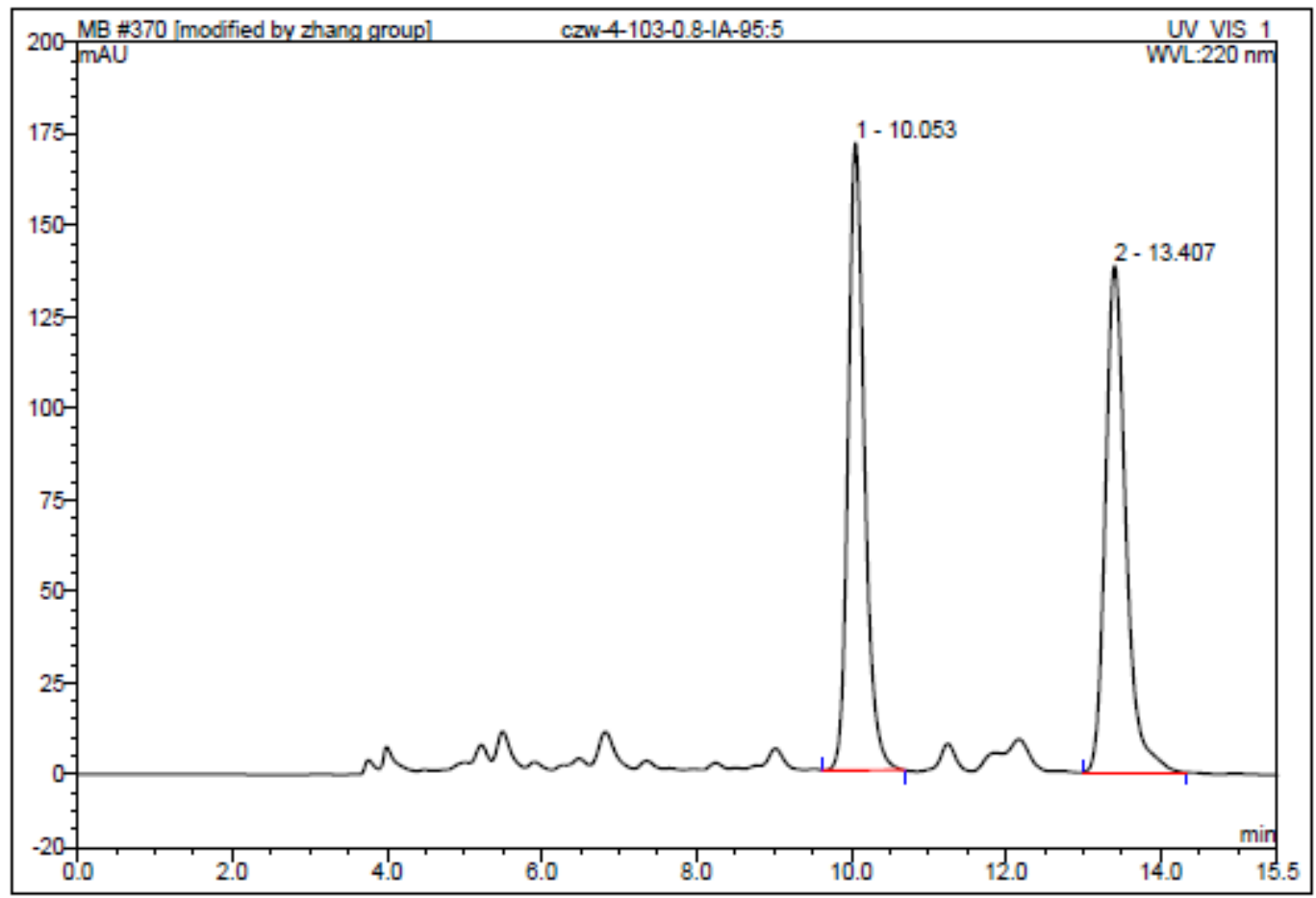

\begin{tabular}{|r|cccrrrr|}
\hline No. & $\begin{array}{c}\text { Ret.Time } \\
\text { min }\end{array}$ & Peak Name & $\begin{array}{c}\text { Height } \\
\text { mAU }\end{array}$ & $\begin{array}{c}\text { Area } \\
\text { mAU*min }\end{array}$ & $\begin{array}{r}\text { Rel.Area } \\
\%\end{array}$ & Amount & Type \\
\hline 1 & 10.05 & n.a. & 171.357 & 42.021 & 49.81 & n.a. & BMB $^{\star}$ \\
2 & 13.41 & n.a. & 138.323 & 42.337 & 50.19 & n.a. & BMB $^{\star}$ \\
\hline Total: & & & 309.681 & 84.358 & 100.00 & 0.000 & \\
\hline
\end{tabular}

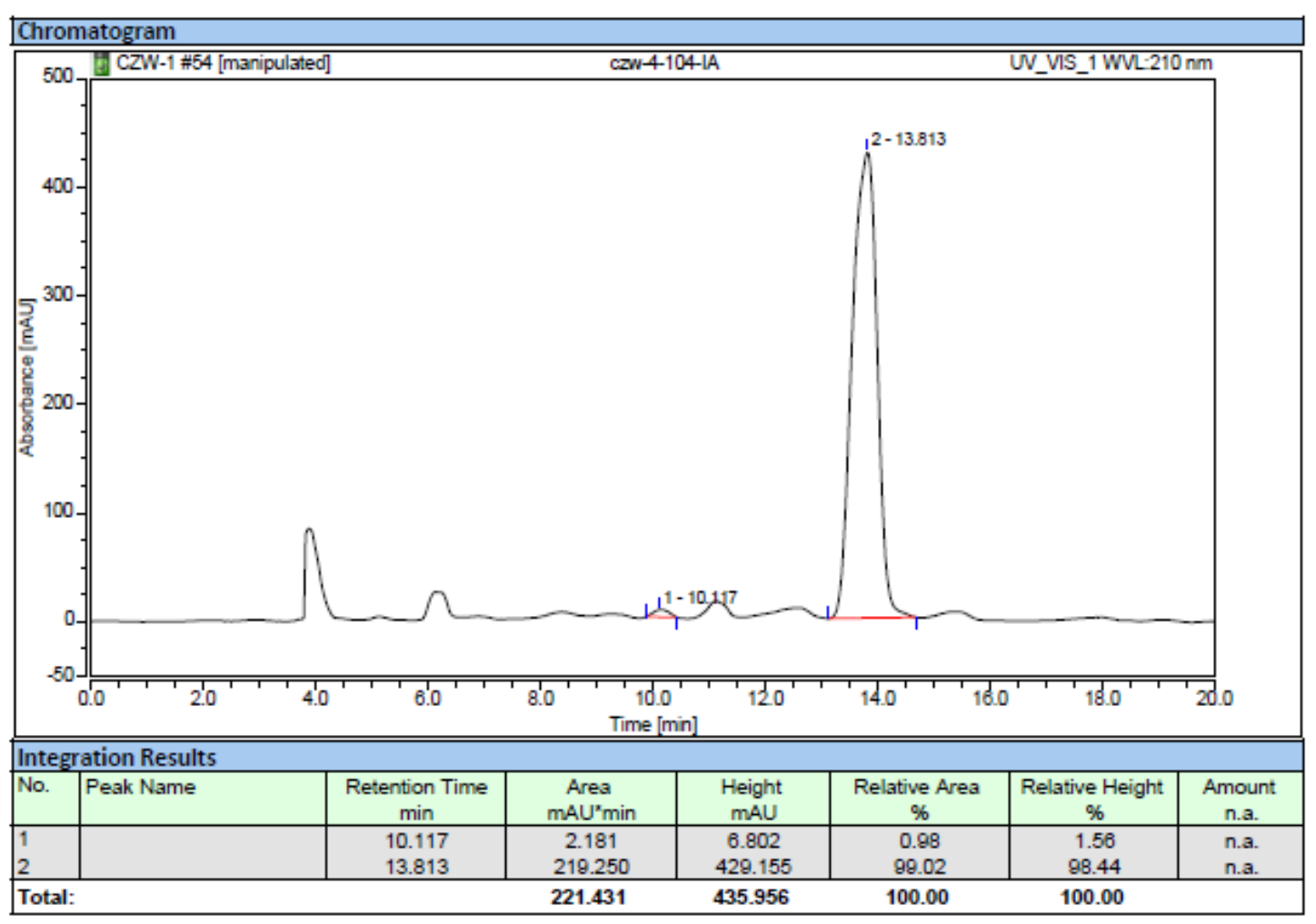

Figure S48: HPLC chromatographs of 3at 


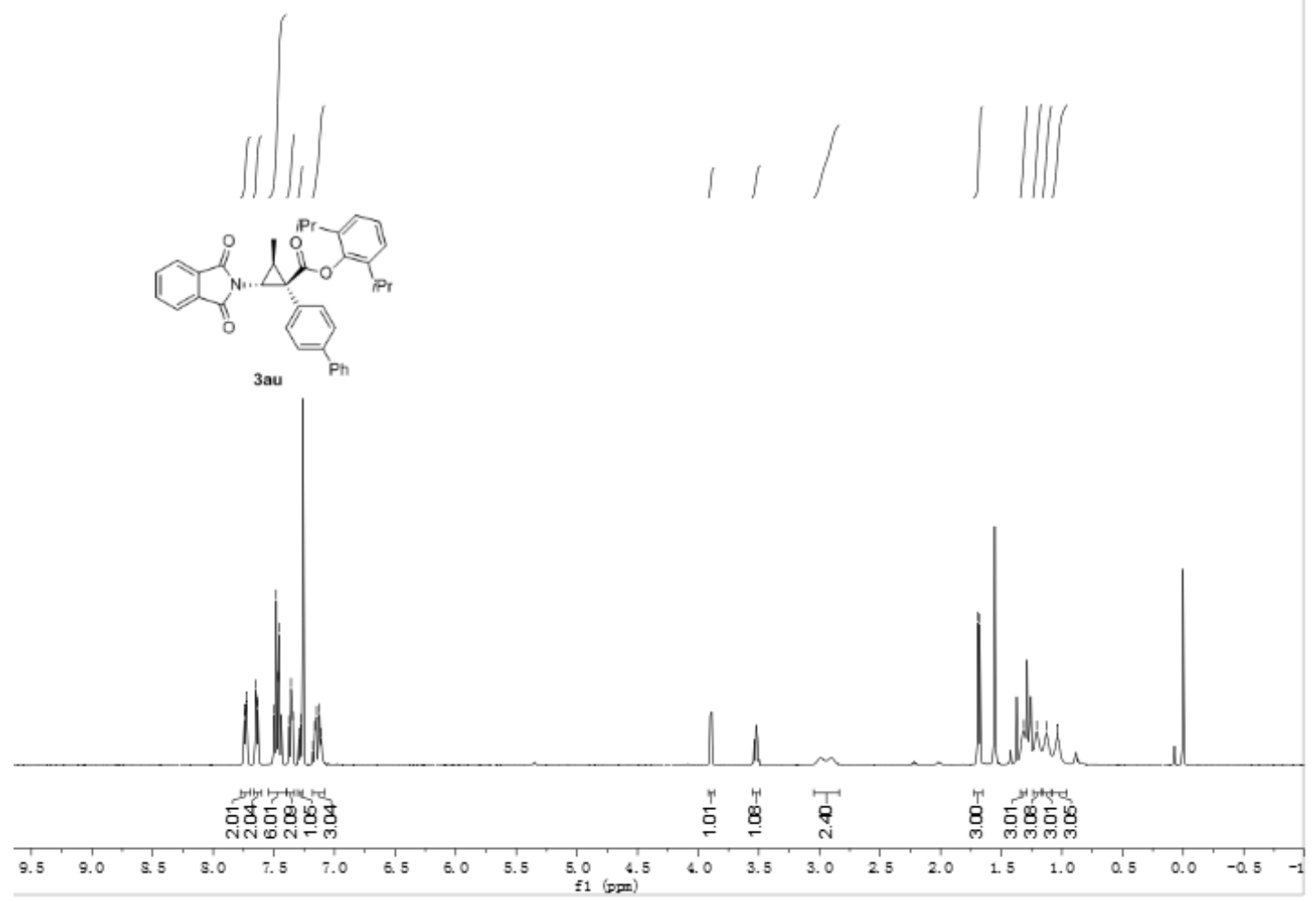

Figure S49: ${ }^{1} \mathrm{H}$ NMR (500 MHz, $\left.\mathrm{CDCl}_{3}\right)$ spectrum for 3au

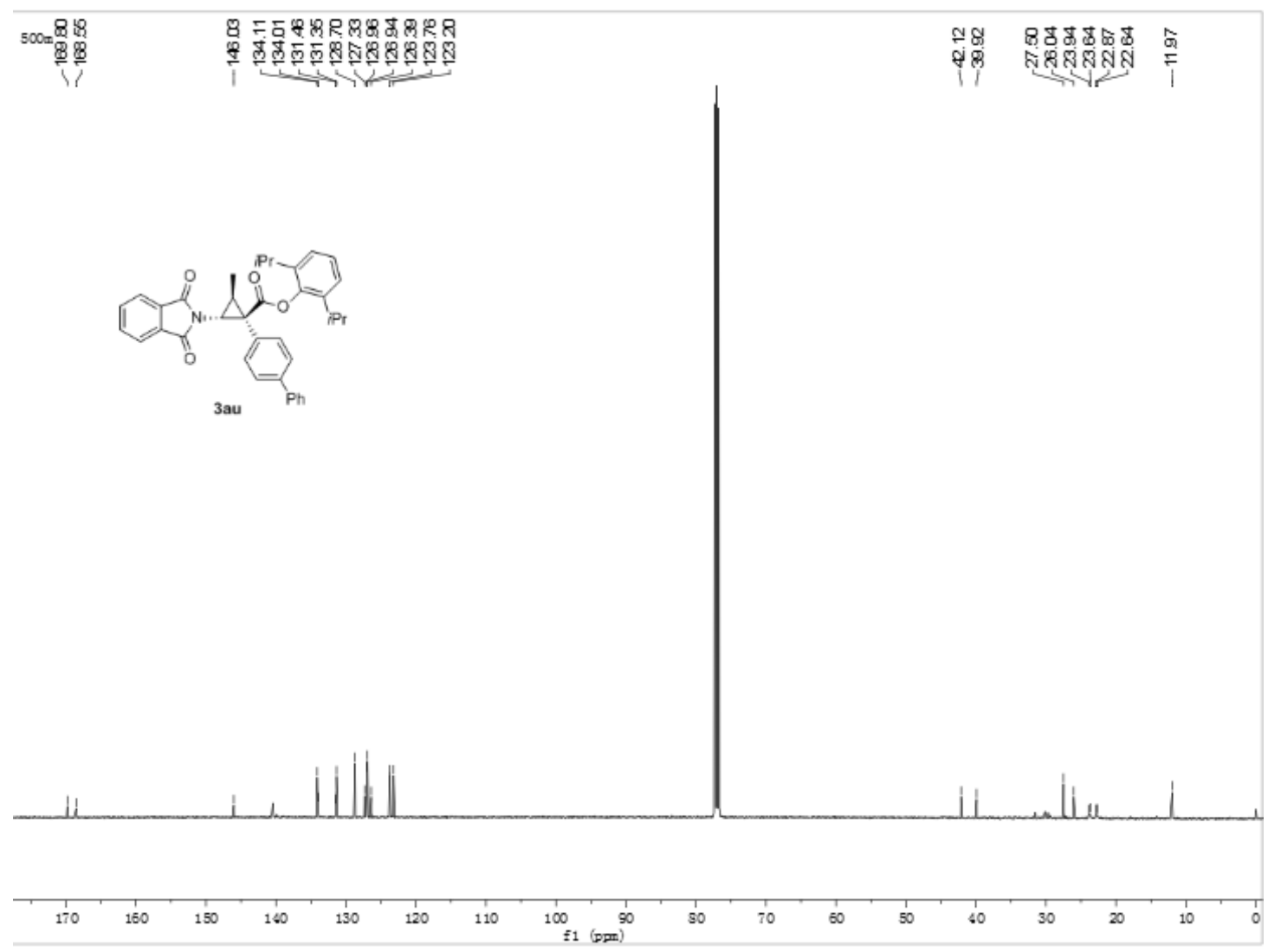

Figure S50: ${ }^{13} \mathrm{C}$ NMR $\left(125 \mathrm{MHz}, \mathrm{CDCl}_{3}\right)$ spectrum for 3au 

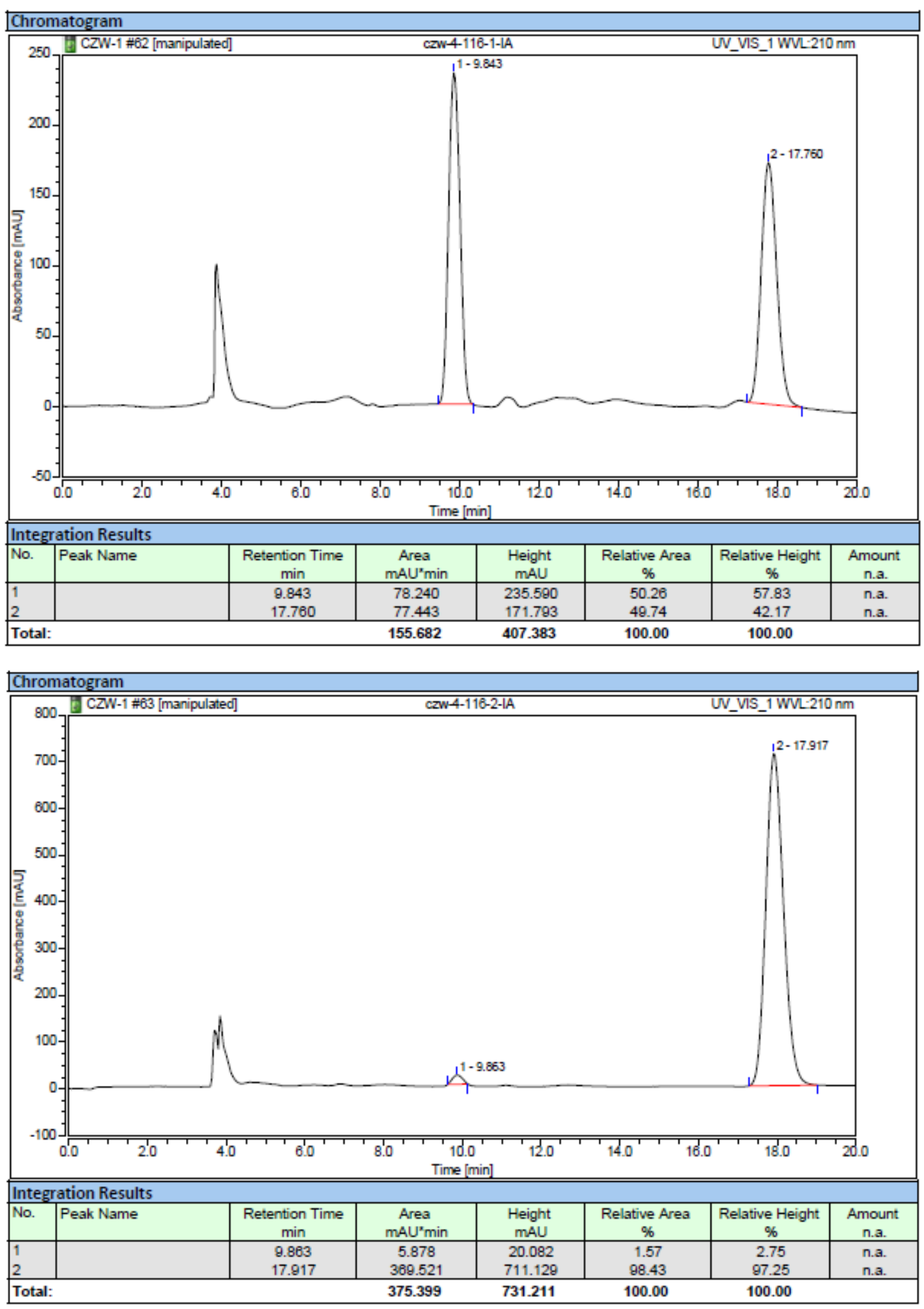

Figure S51: HPLC chromatographs of 3au 


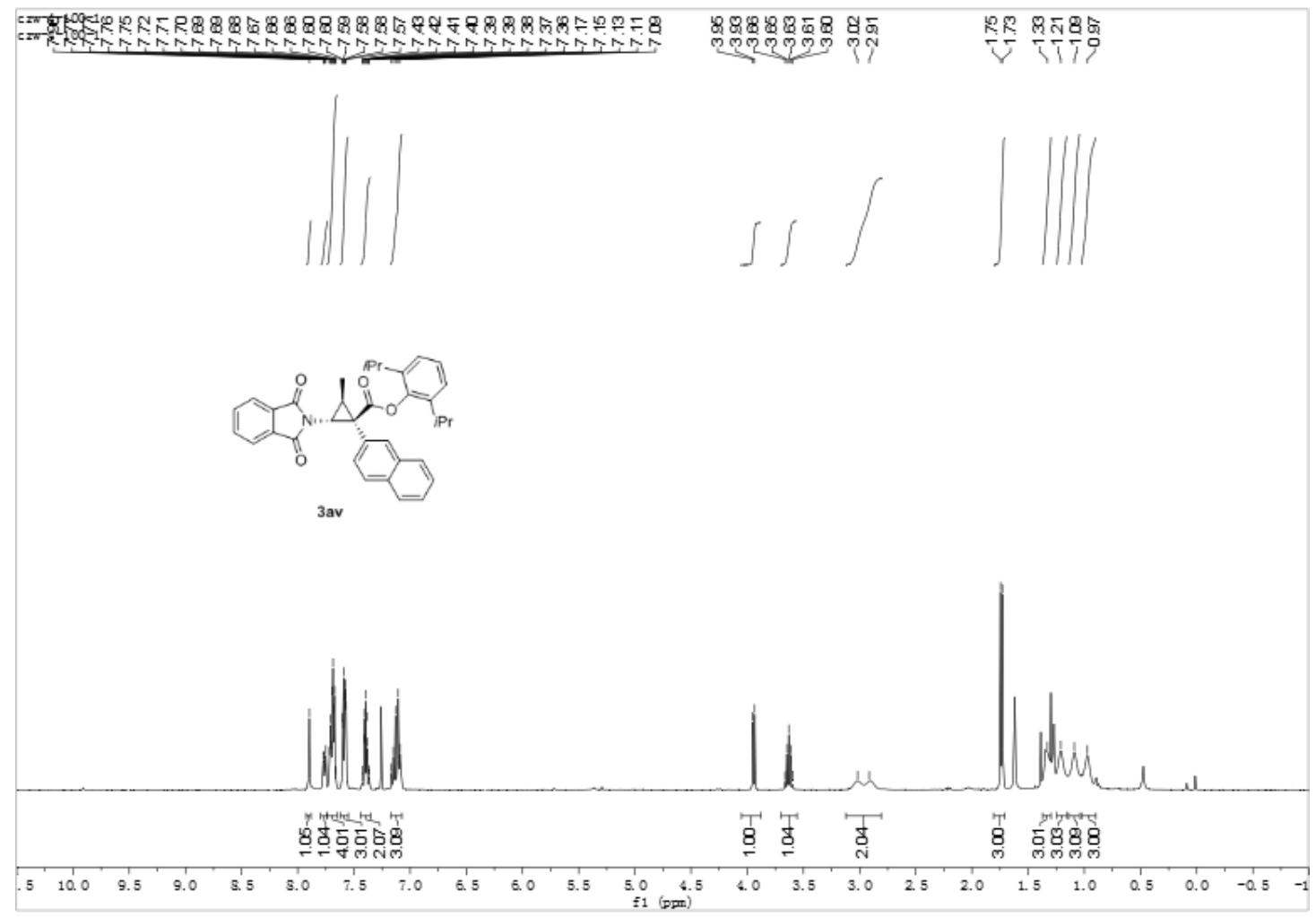

Figure S52: ${ }^{1} \mathrm{H}$ NMR (400 MHz, $\left.\mathrm{CDCl}_{3}\right)$ spectrum for 3av

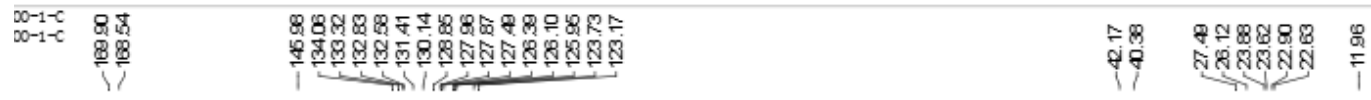

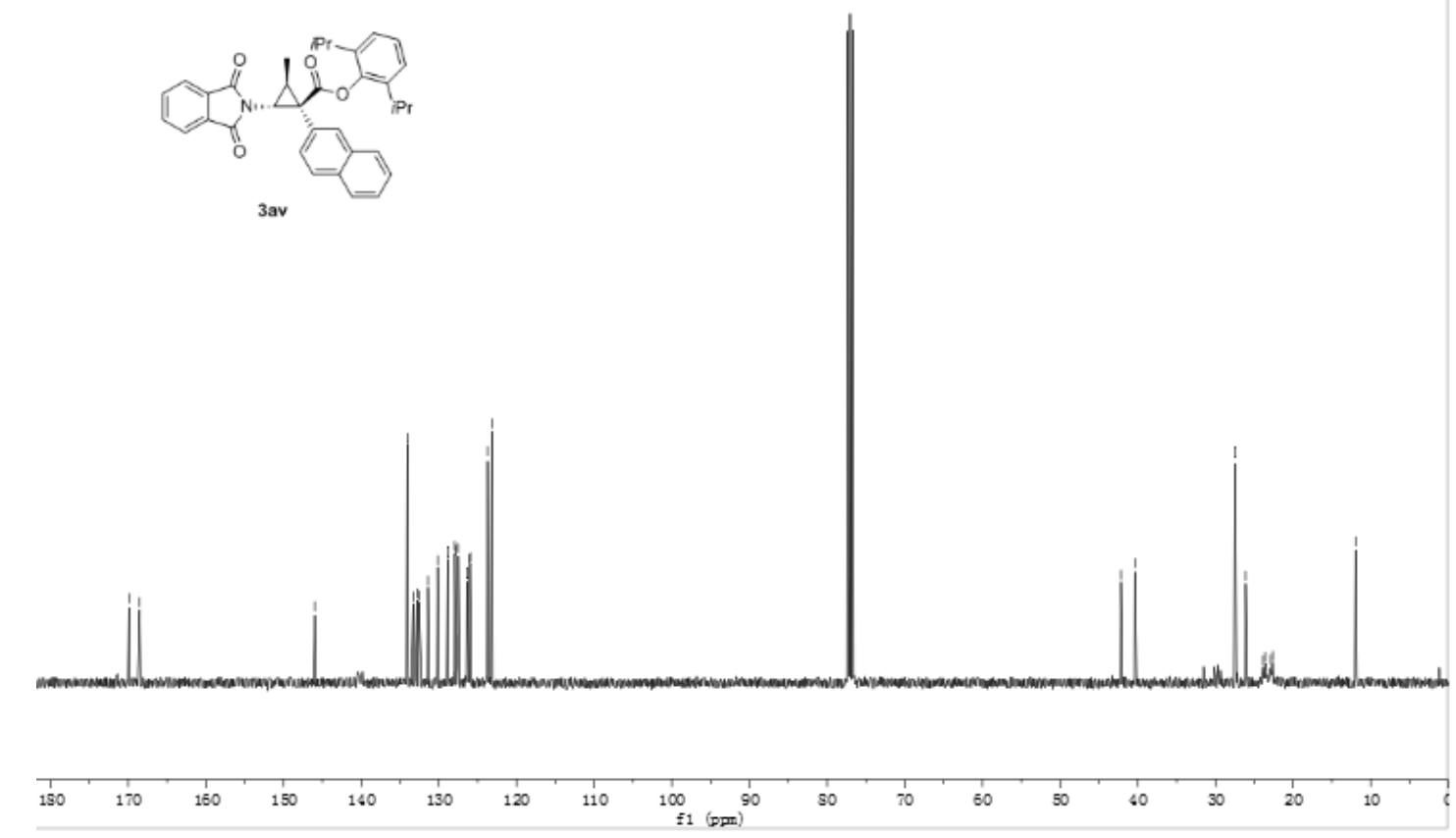

3av

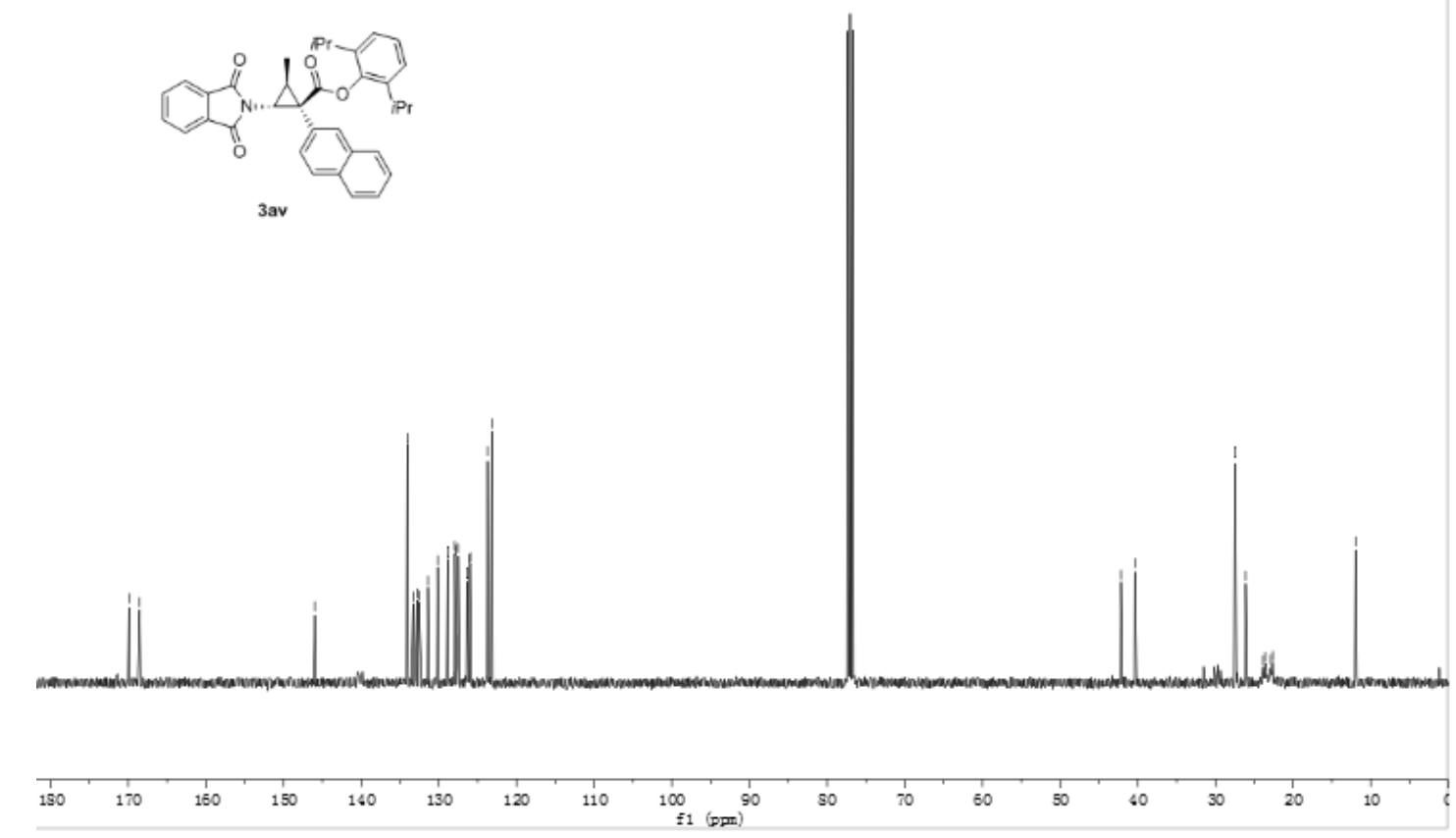

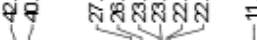

Figure S53: ${ }^{13} \mathrm{C}$ NMR $\left(100 \mathrm{MHz}, \mathrm{CDCl}_{3}\right)$ spectrum for 3av 

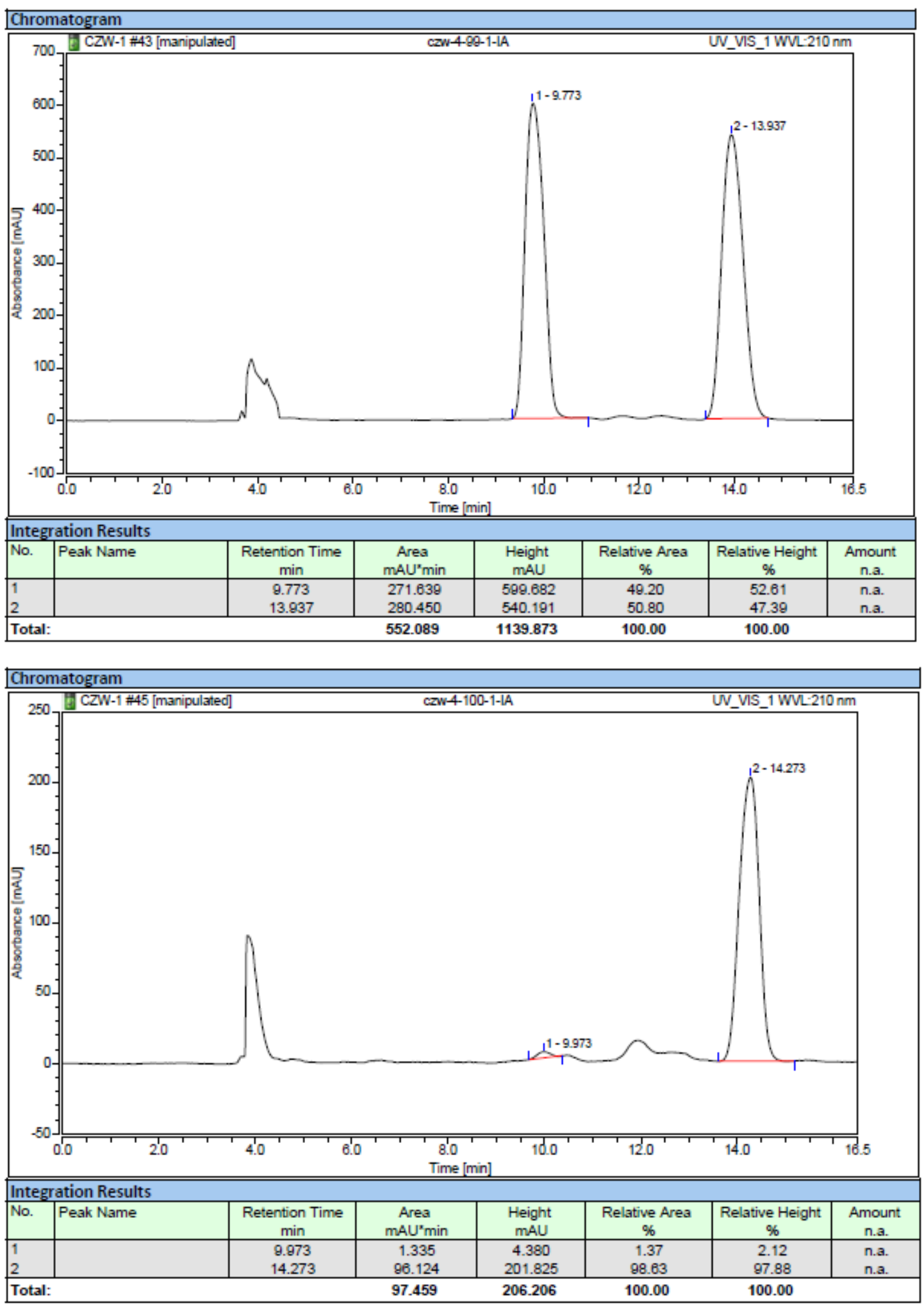

Figure S54: HPLC chromatographs of 3av 


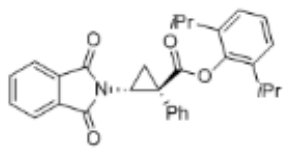

3 be

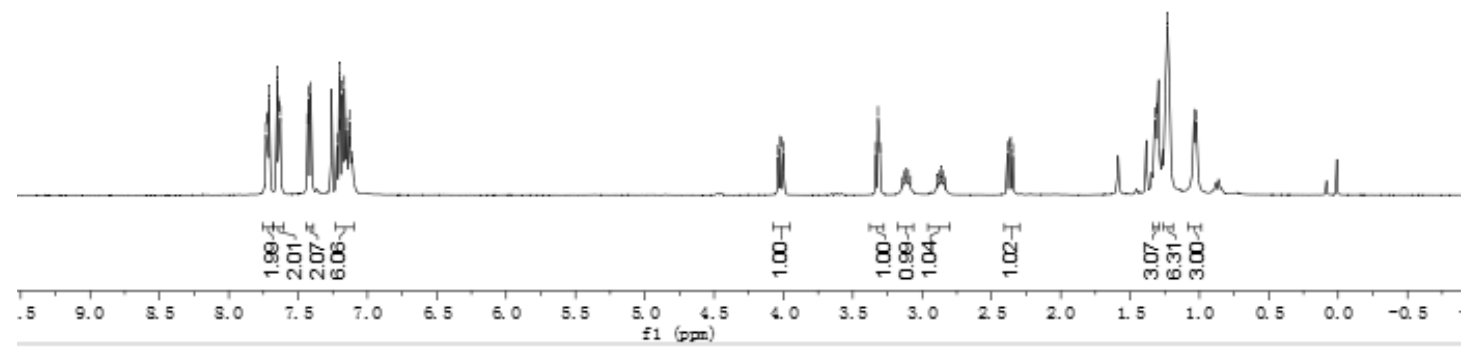

Figure S55: ${ }^{1} \mathrm{H}$ NMR (400 MHz, $\left.\mathrm{CDCl}_{3}\right)$ spectrum for $3 \mathbf{b e}$

\begin{tabular}{|c|c|c|}
\hline $\begin{array}{l}130-2 \\
130-2=\frac{18}{10}\end{array}$ & 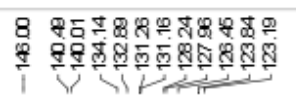 & 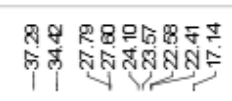 \\
\hline
\end{tabular}
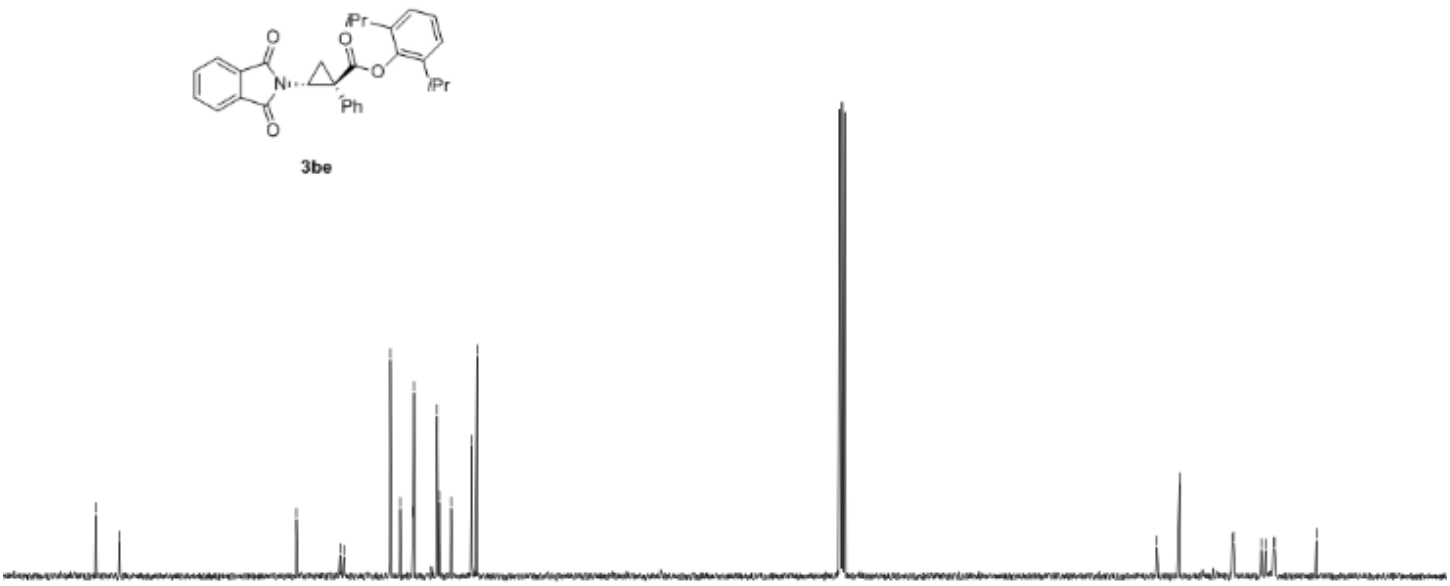

Figure S56: ${ }^{13} \mathrm{C} \mathrm{NMR}\left(100 \mathrm{MHz}, \mathrm{CDCl}_{3}\right)$ spectrum for 3ae 

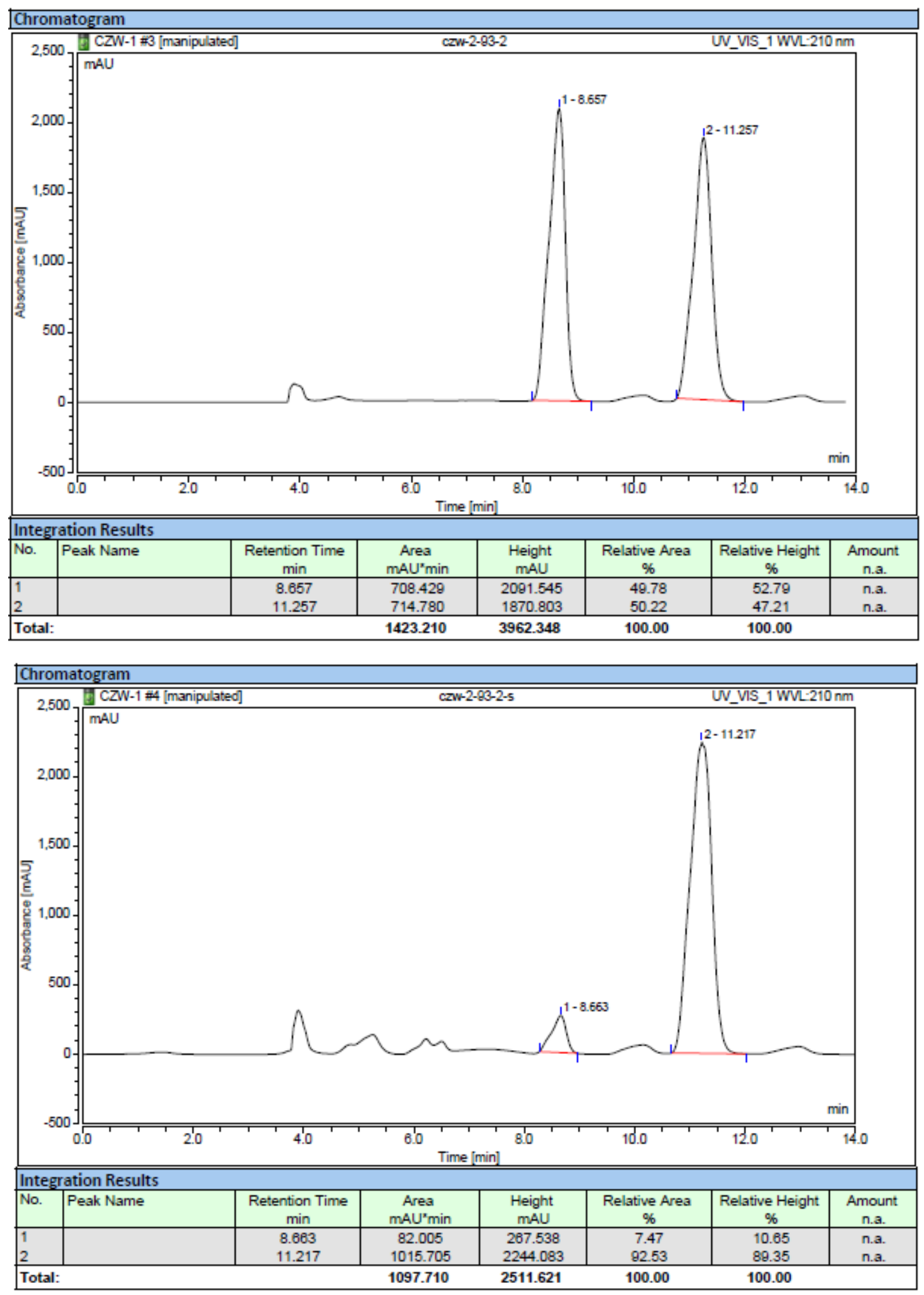

Figure S57: HPLC chromatographs of $3 \mathbf{b e}$ 


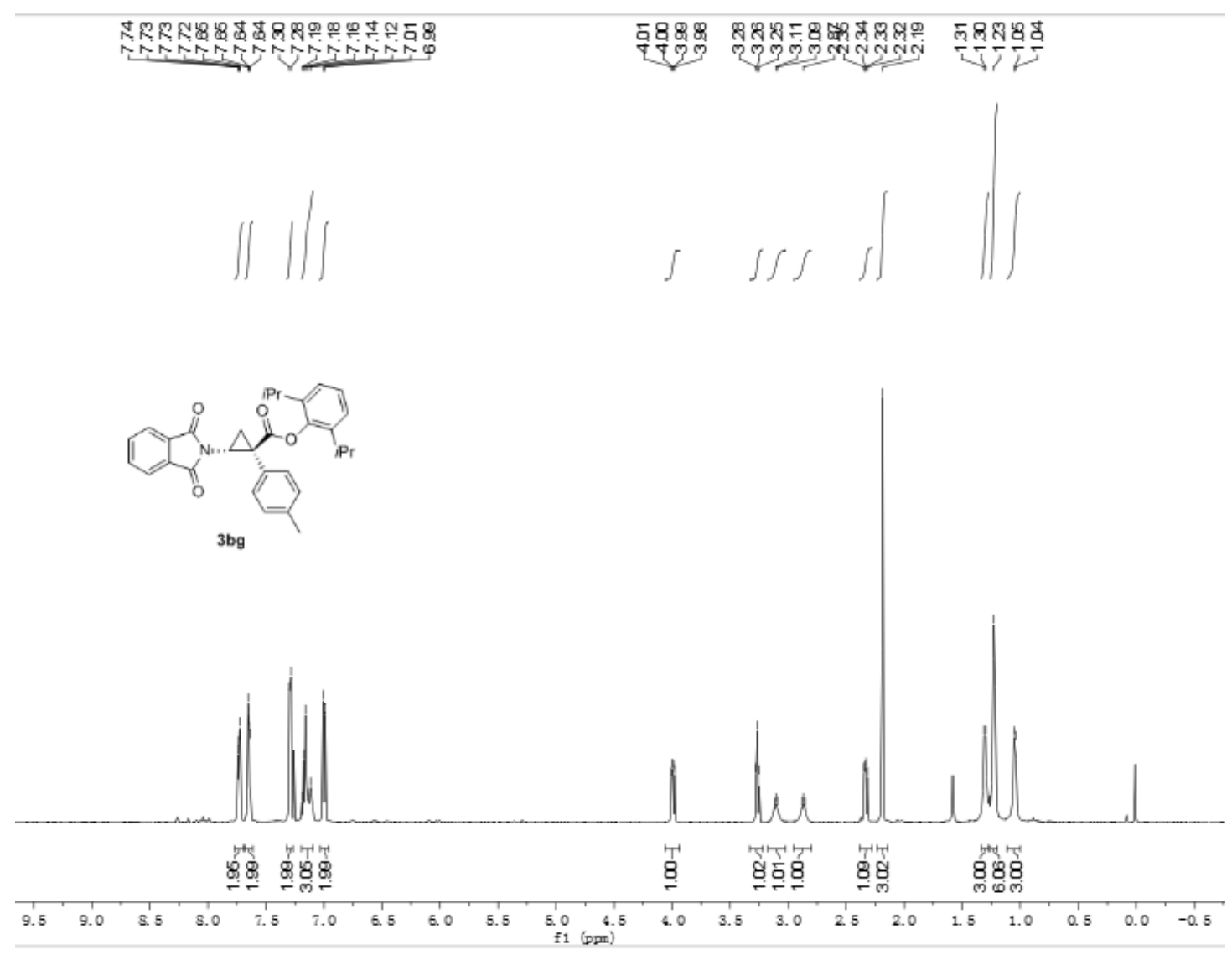

Figure S58: ${ }^{1} \mathrm{H} \mathrm{NMR}\left(500 \mathrm{MHz}, \mathrm{CDCl}_{3}\right)$ spectrum for $\mathbf{3 b g}$

\begin{tabular}{|c|c|}
\hline$=\frac{\mathscr{R} F}{5}$ & 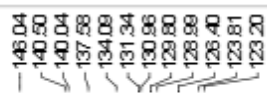 \\
\hline
\end{tabular}

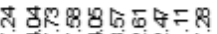

ठิ

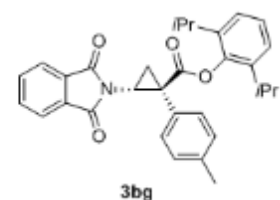

$3 \mathrm{bg}$
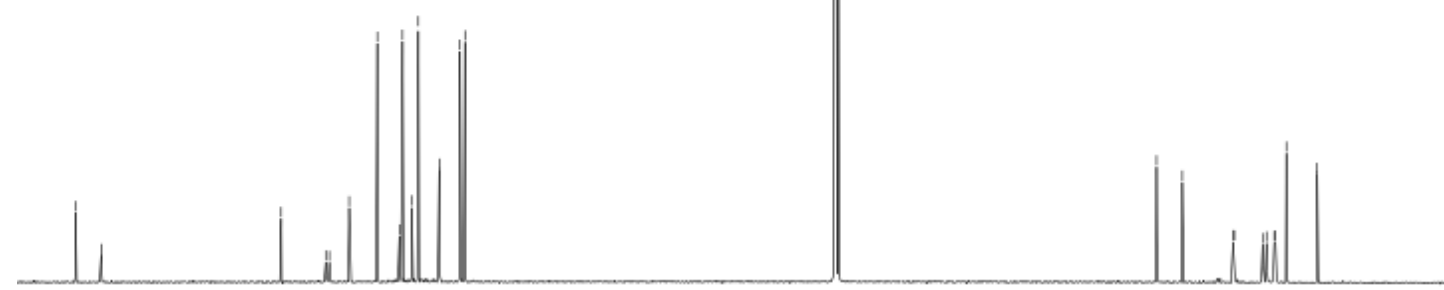

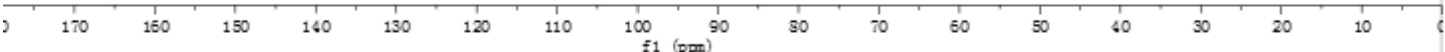

Figure S59: ${ }^{13} \mathrm{C}$ NMR $\left(125 \mathrm{MHz}, \mathrm{CDCl}_{3}\right)$ spectrum for $\mathbf{3 b g}$ 


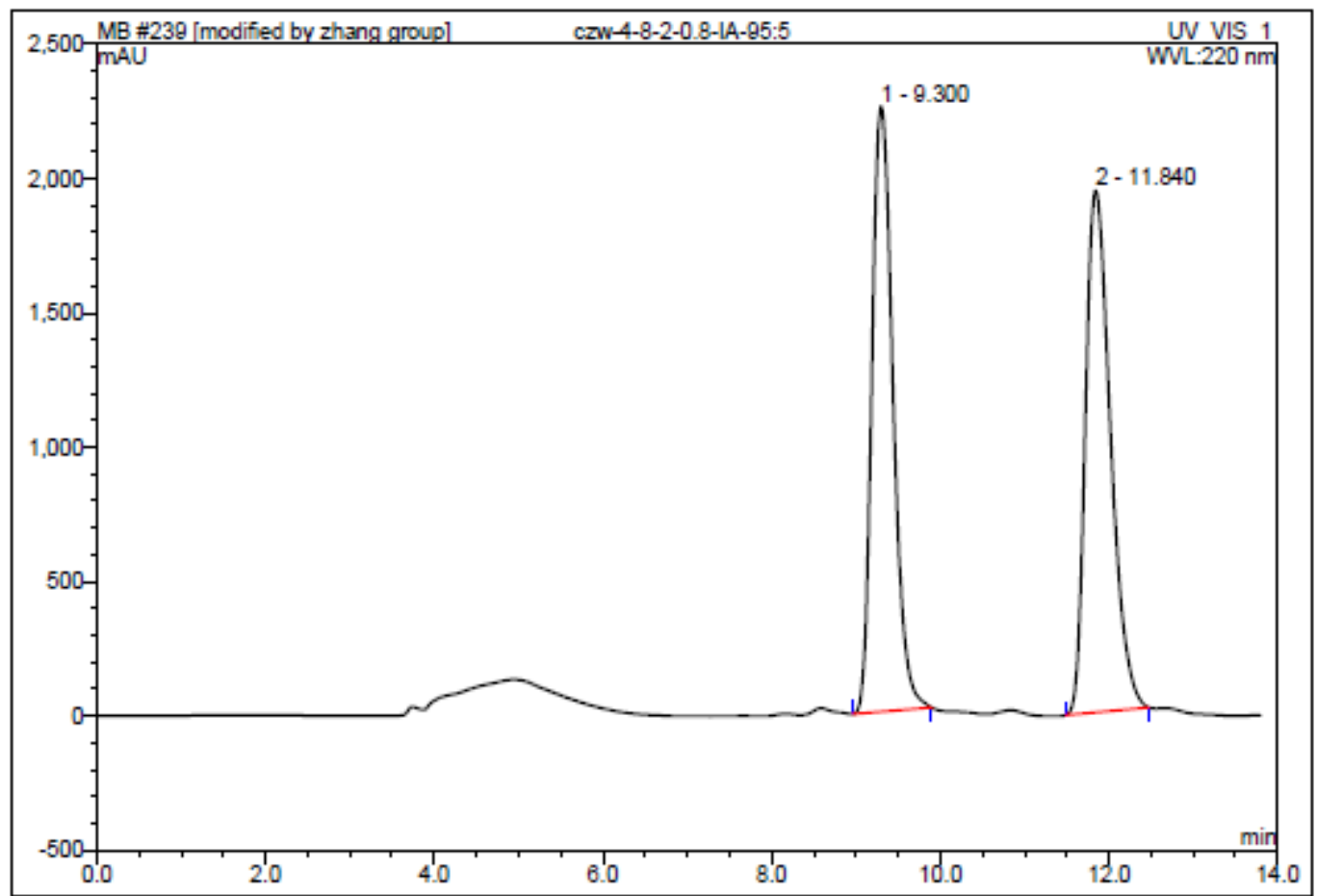

\begin{tabular}{|r|rrrrrrr|}
\hline No. & $\begin{array}{c}\text { Ret.Time } \\
\text { min }\end{array}$ & Peak Name & $\begin{array}{c}\text { Height } \\
\text { mAU }\end{array}$ & $\begin{array}{c}\text { Area } \\
\text { mAU*min }\end{array}$ & $\begin{array}{r}\text { Rel.Area } \\
\%\end{array}$ & Amount & Type \\
\hline 1 & 9.30 & n.a. & 2253.402 & 650.753 & 49.35 & n.a. & BMB $^{\star}$ \\
2 & 11.84 & n.a. & 1941.549 & 668.014 & 50.65 & n.a. & BMB $^{\star}$ \\
\hline Total: & & & 4194.951 & 1318.767 & 100.00 & 0.000 & \\
\hline
\end{tabular}

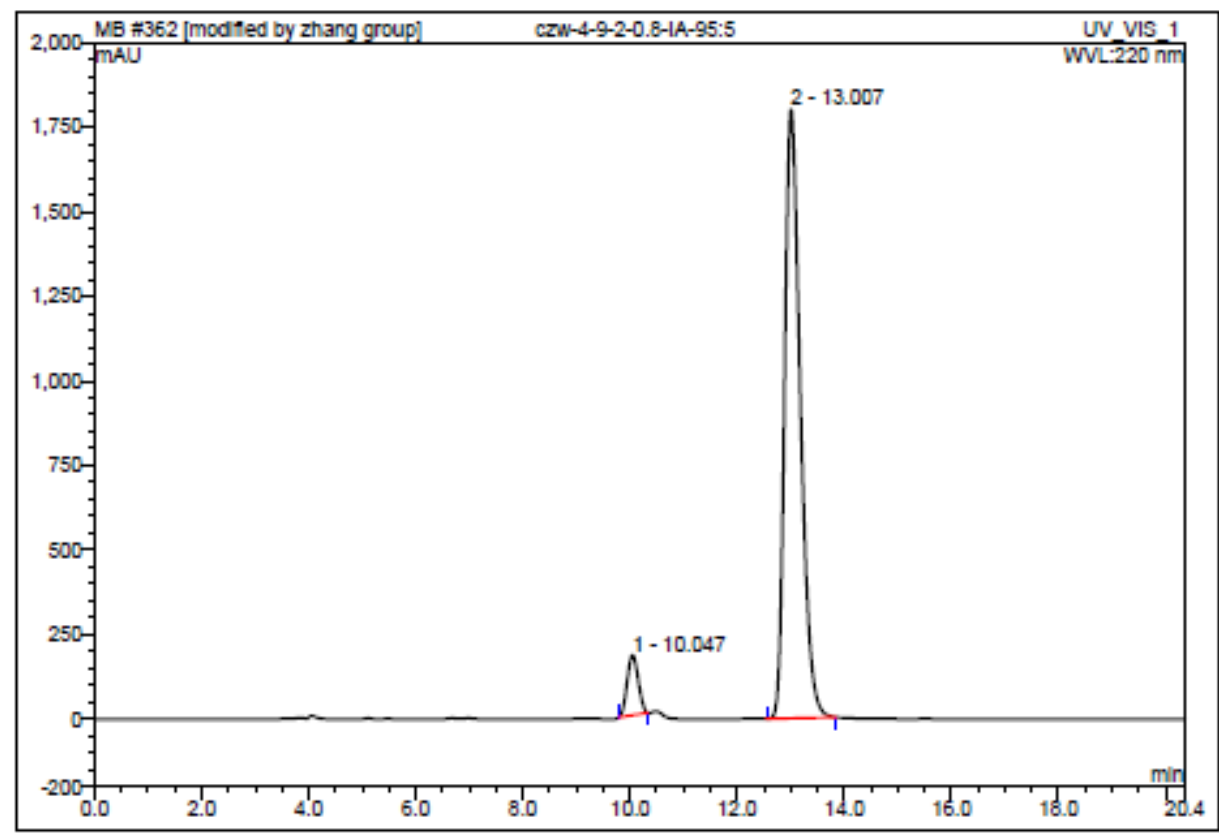

\begin{tabular}{|r|ccrrrrr|}
\hline No. & $\begin{array}{c}\text { Ret.Time } \\
\text { min }\end{array}$ & Peak Name & $\begin{array}{c}\text { Height } \\
\text { mAU }\end{array}$ & $\begin{array}{c}\text { Area } \\
\text { mAU*min }\end{array}$ & $\begin{array}{r}\text { Rel.Area } \\
\text { Amount }\end{array}$ & Type \\
\hline 1 & 10.05 & n.a. & 177.460 & 42.561 & 6.47 & n.a. & BMB $^{*}$ \\
2 & 13.01 & n.a. & 1799.865 & 614.973 & 93.53 & n.a. & BMB $^{*}$ \\
\hline Total: & & & 1977.325 & 657.535 & 100.00 & 0.000 & \\
\hline
\end{tabular}

Figure S60: HPLC chromatographs of $\mathbf{3 b g}$ 


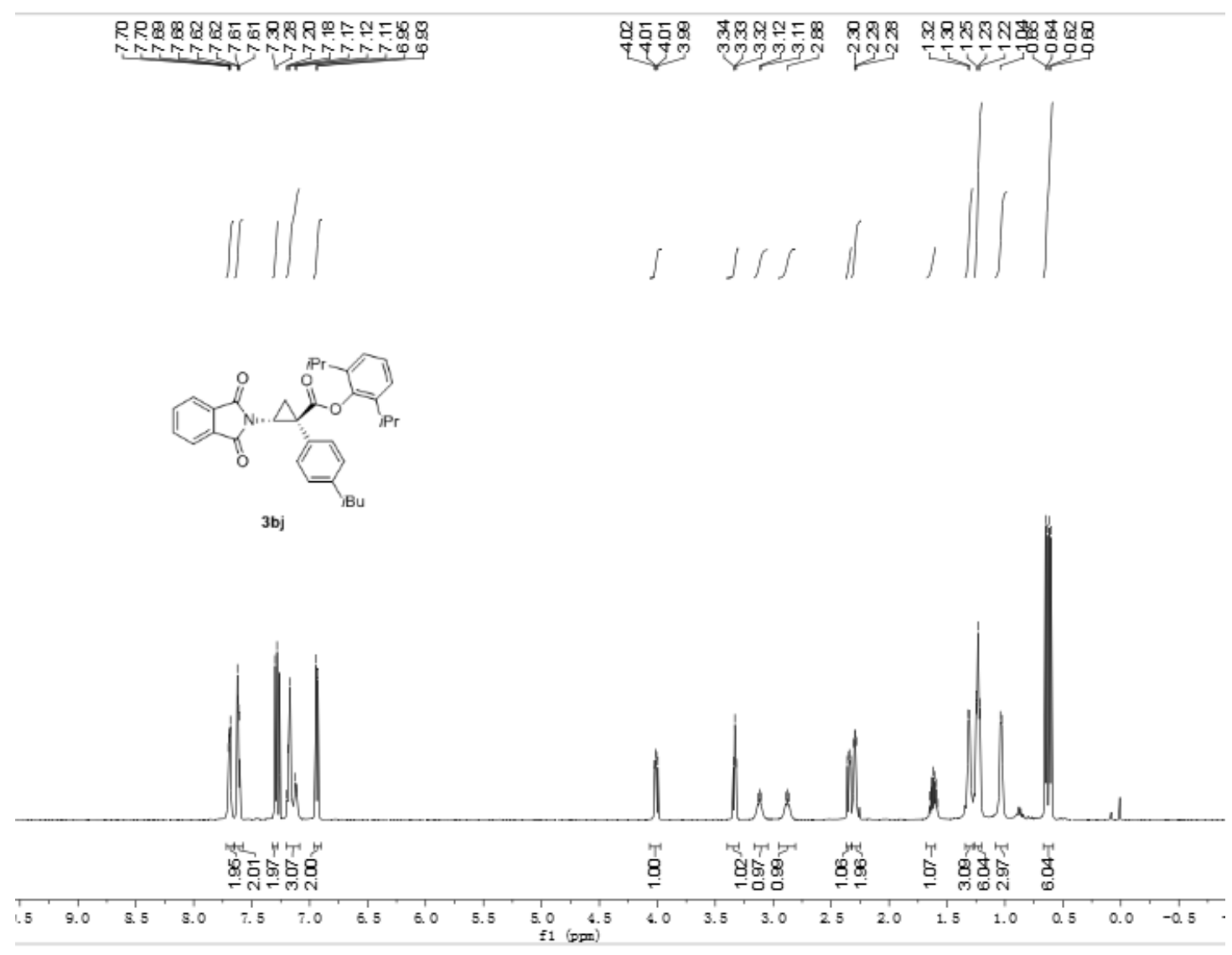

Figure S61: ${ }^{1} \mathrm{H}$ NMR $\left(500 \mathrm{MHz}, \mathrm{CDCl}_{3}\right)$ spectrum for $\mathbf{3 b j}$ 


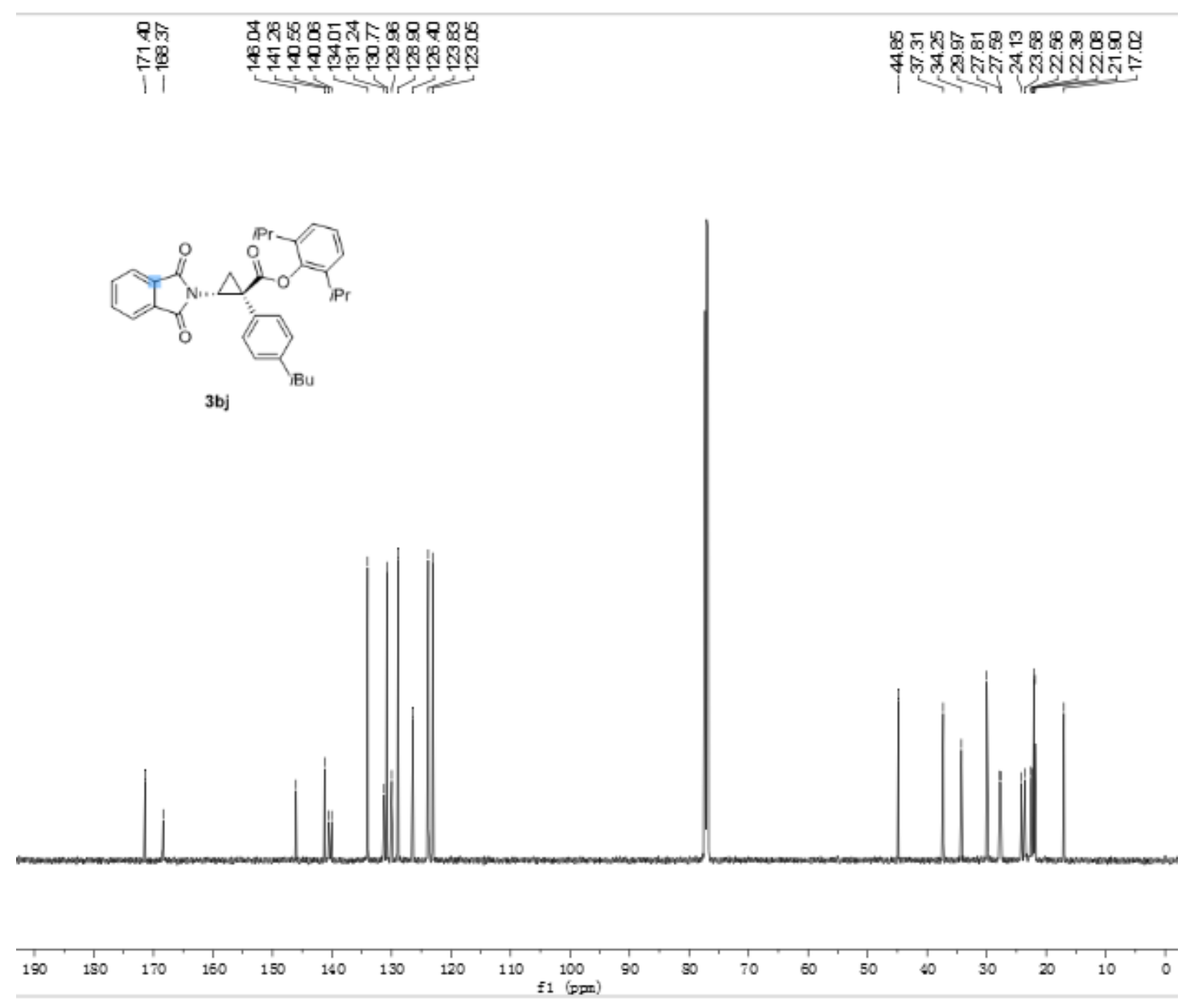

Figure S62: ${ }^{13} \mathrm{C}$ NMR $\left(125 \mathrm{MHz}, \mathrm{CDCl}_{3}\right)$ spectrum for $\mathbf{3 b j}$ 


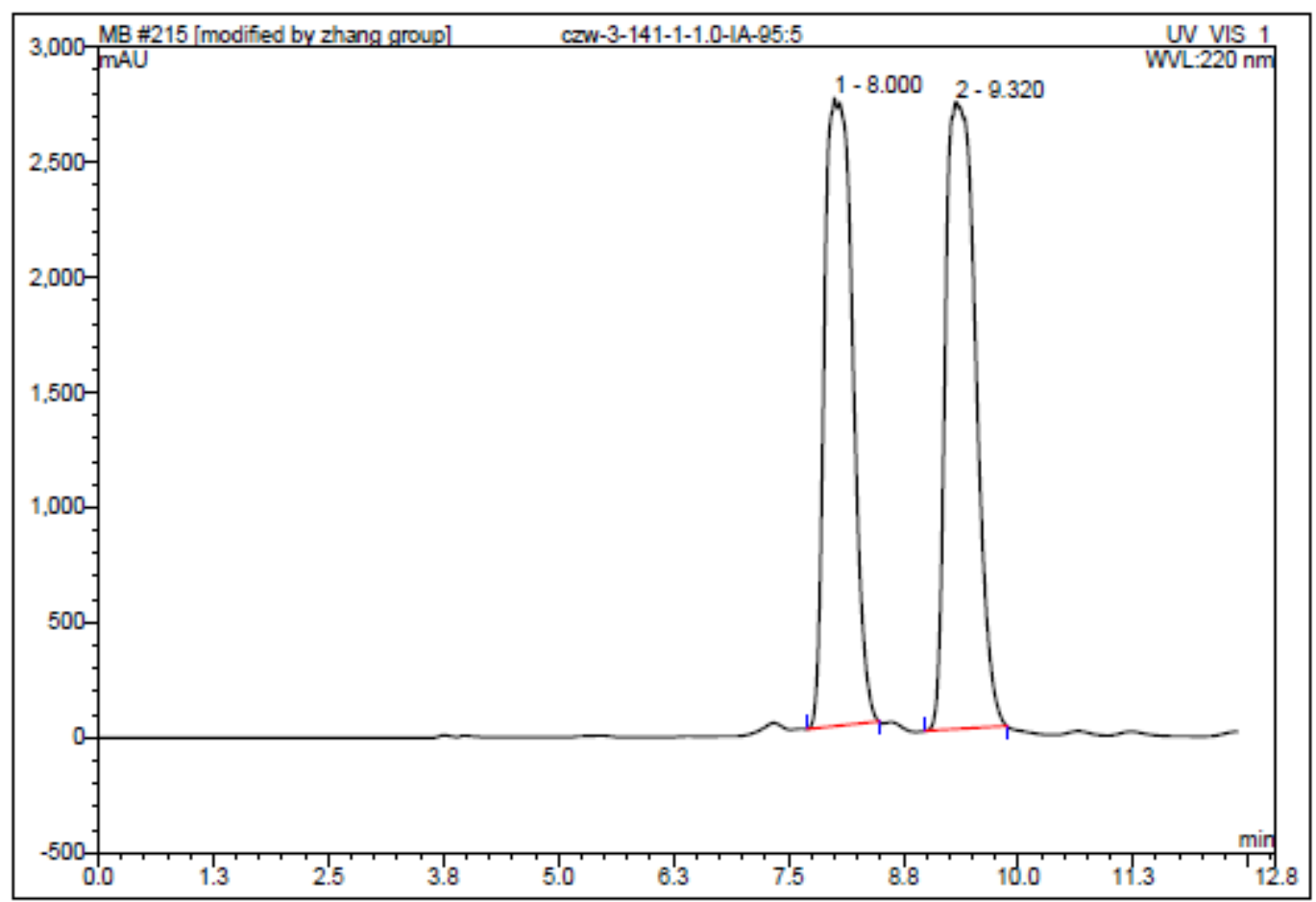

\begin{tabular}{|r|ccrrrrr|}
\hline No. & $\begin{array}{c}\text { Ret.Time } \\
\text { min }\end{array}$ & Peak Name & $\begin{array}{c}\text { Height } \\
\text { mAU }\end{array}$ & $\begin{array}{c}\text { Area } \\
\text { mAU*min }\end{array}$ & $\begin{array}{c}\text { Rel.Area } \\
\%\end{array}$ & Amount & Type \\
\hline 1 & 8.00 & n.a. & 2730.351 & 961.342 & 48.18 & n.a. & BMB $^{\star}$ \\
2 & 9.32 & n.a. & 2727.228 & 1033.904 & 51.82 & n.a. & BMB $^{\star}$ \\
\hline Total: & & & 5457.579 & 1995.246 & 100.00 & 0.000 & \\
\hline
\end{tabular}

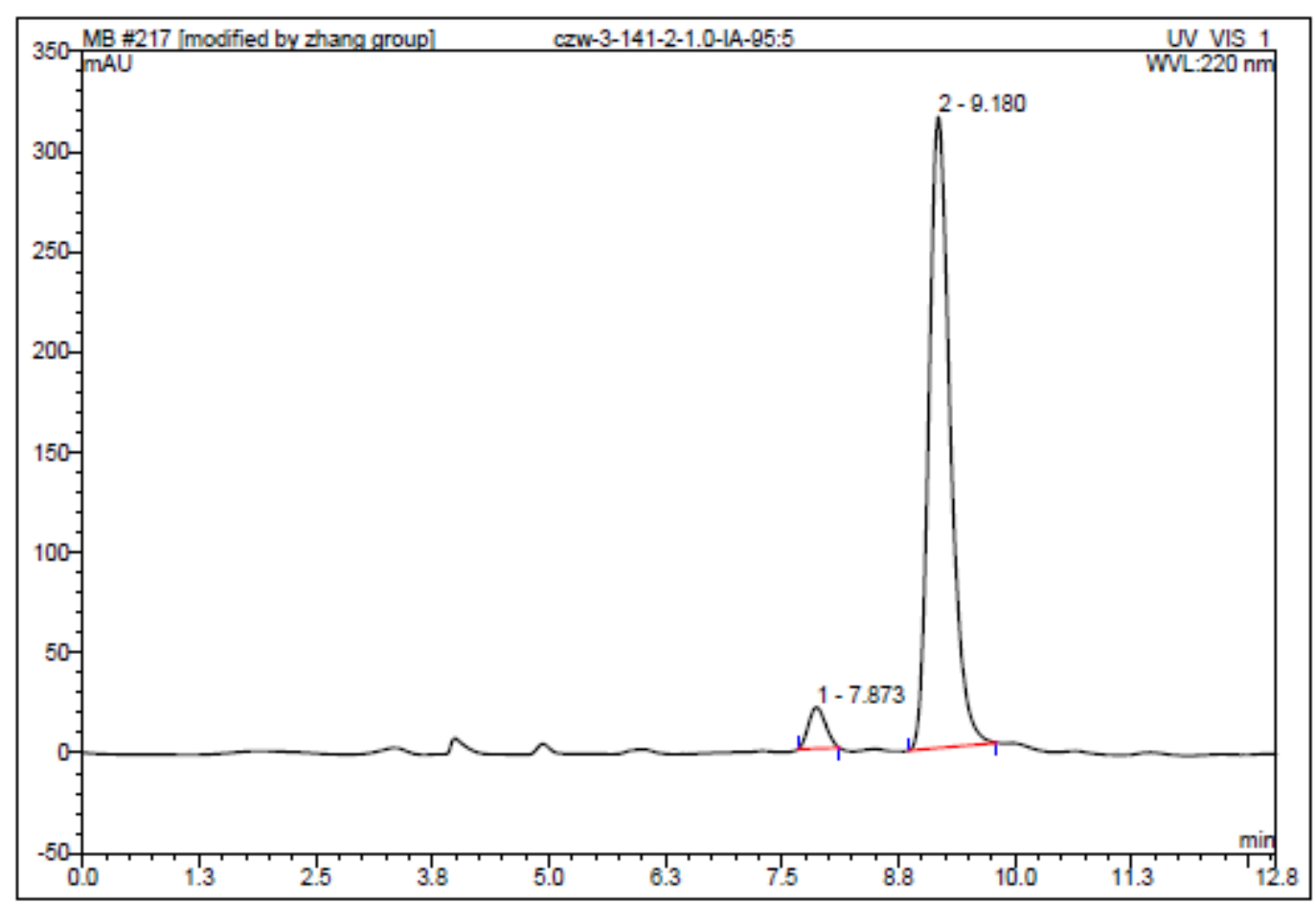

\begin{tabular}{|r|ccrrrrr|}
\hline No. & $\begin{array}{c}\text { Ret.Time } \\
\text { min }\end{array}$ & Peak Name & $\begin{array}{c}\text { Height } \\
\text { mAU }\end{array}$ & $\begin{array}{c}\text { Area } \\
\text { mAU*min }\end{array}$ & $\begin{array}{r}\text { Rel.Area } \\
\%\end{array}$ & Amount & Type \\
\hline 1 & 7.87 & n.a. & 20.475 & 4.372 & 4.98 & n.a. & BMB $^{\star}$ \\
2 & 9.18 & n.a. & 314.691 & 83.461 & 95.02 & n.a. & BMB $^{\star}$ \\
\hline Total: & & & 335.166 & 87.833 & 100.00 & 0.000 & \\
\hline
\end{tabular}


Figure S63: HPLC chromatographs of $\mathbf{3 b j}$

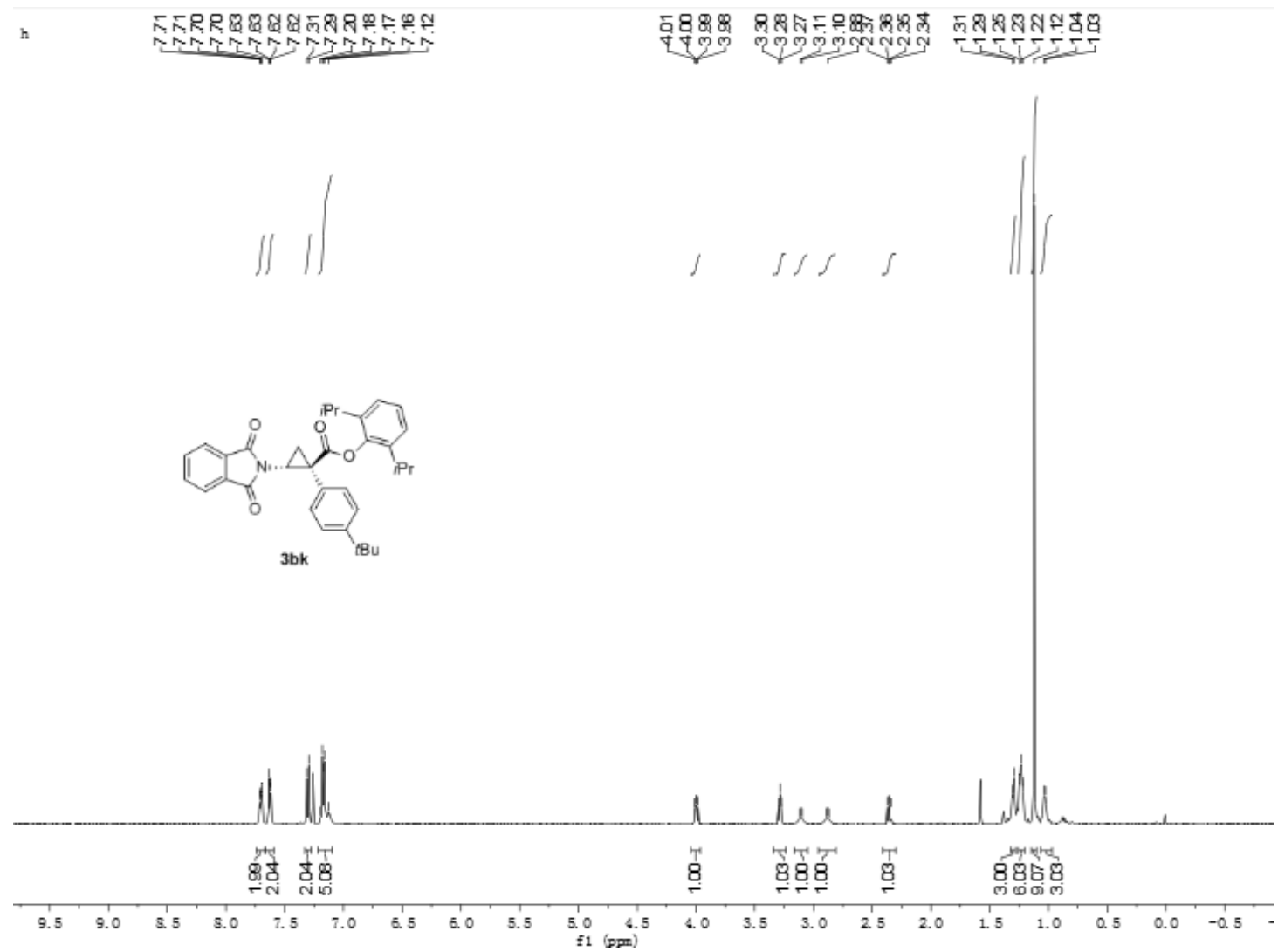

Figure S64: ${ }^{1} \mathrm{H}$ NMR (500 MHz, $\mathrm{CDCl}_{3}$ ) spectrum for $\mathbf{3 b k}$

\begin{tabular}{|c|c|c|}
\hline $1=\frac{87}{1}$ & 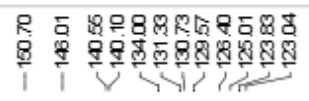 & 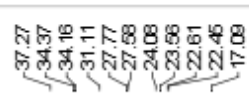 \\
\hline
\end{tabular}
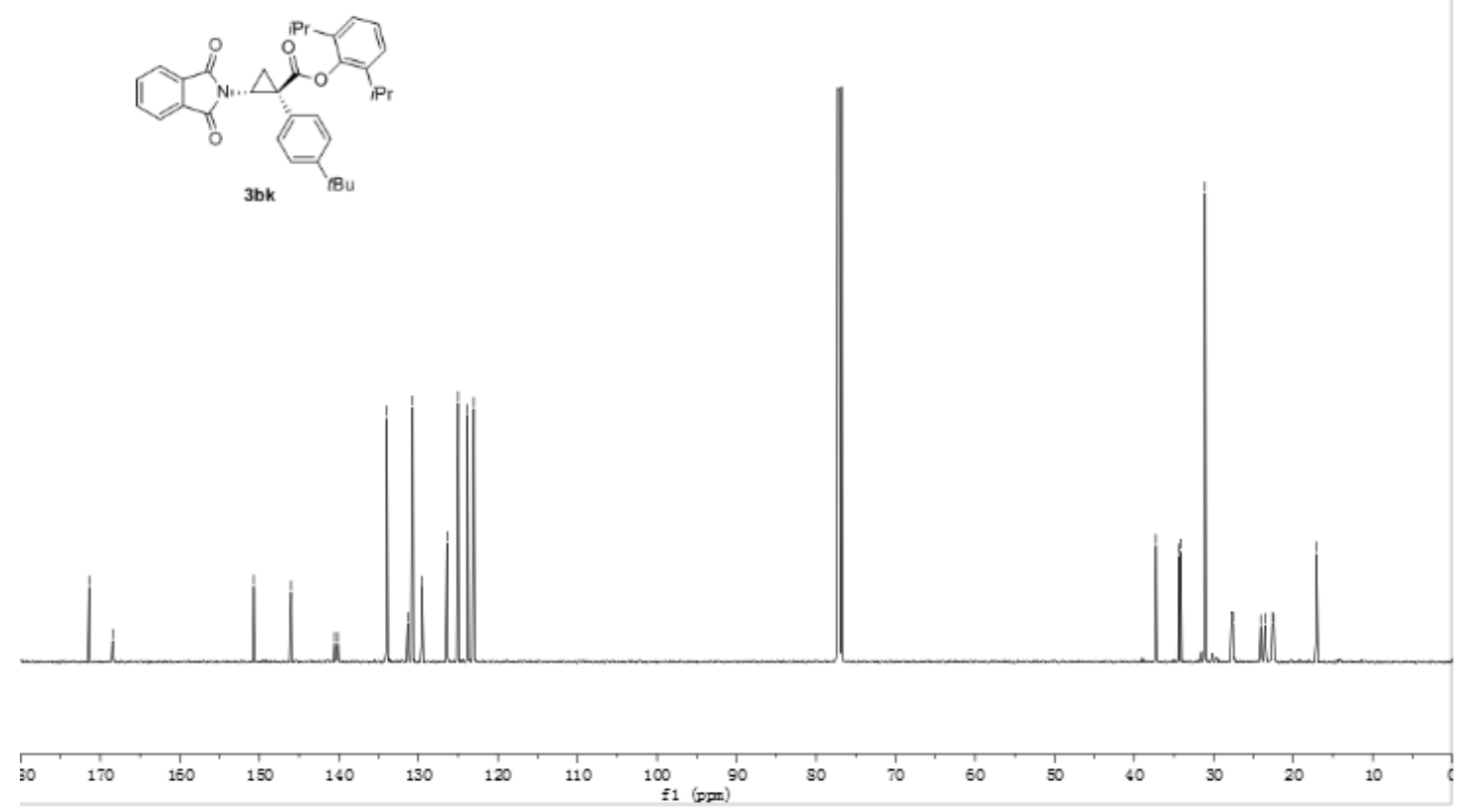

Figure S65: ${ }^{13} \mathrm{C}$ NMR $\left(125 \mathrm{MHz}, \mathrm{CDCl}_{3}\right)$ spectrum for $\mathbf{3 b k}$ 


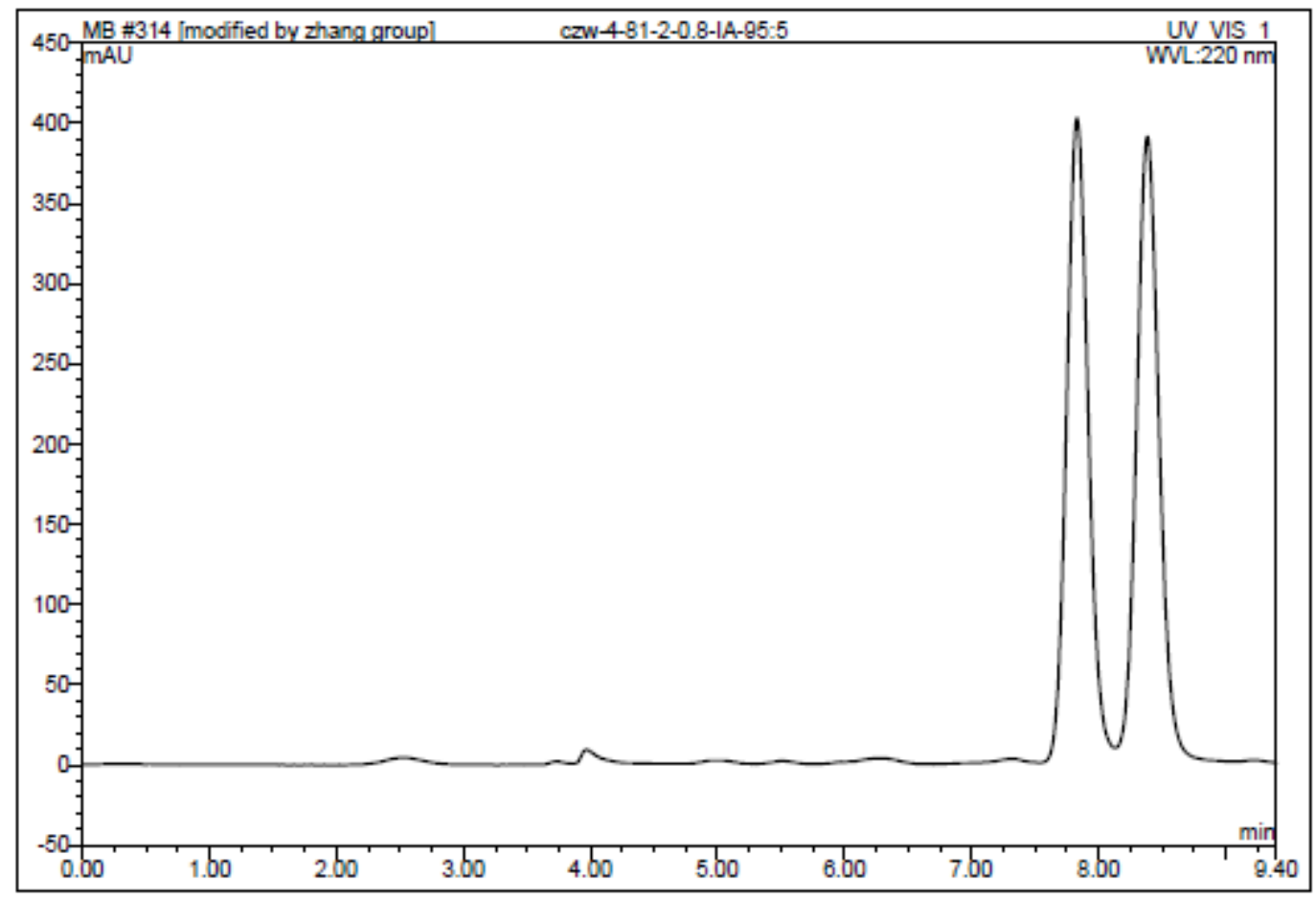

\begin{tabular}{|c|ccccccc|}
\hline No. & $\begin{array}{c}\text { Ret.Time } \\
\mathrm{min}\end{array}$ & Peak Name & $\begin{array}{c}\text { Height } \\
\mathrm{mAU}\end{array}$ & $\begin{array}{c}\text { Area } \\
\mathrm{mAU} \mathrm{min}^{*}\end{array}$ & $\begin{array}{c}\text { Rel.Area } \\
\%\end{array}$ & Amount & Type \\
\hline Total: & & & 0.000 & 0.000 & 0.00 & 0.000 & \\
\hline
\end{tabular}

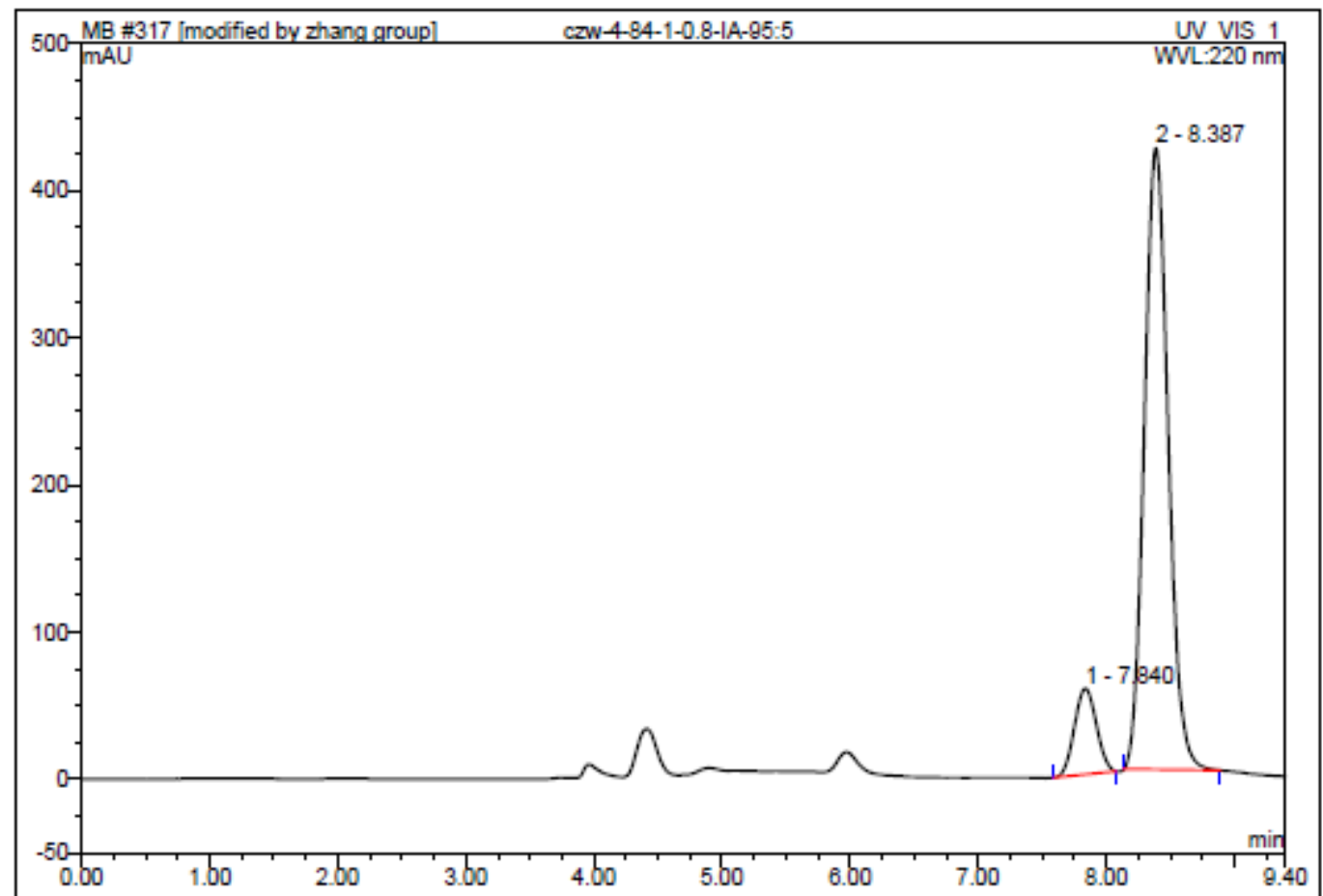

\begin{tabular}{|c|c|c|c|c|c|c|c|}
\hline No. & $\begin{array}{c}\text { Ret.Time } \\
\text { min }\end{array}$ & Peak Name & $\begin{array}{c}\text { Height } \\
\text { mAU }\end{array}$ & $\begin{array}{c}\text { Area } \\
\mathrm{mAU}^{*} \min \end{array}$ & $\begin{array}{c}\text { Rel.Area } \\
\% \\
\end{array}$ & Amount & Type \\
\hline 1 & 7.84 & n.a. & 58.484 & 11.588 & 11.31 & n.a. & $\mathrm{BMB}^{\star}$ \\
\hline 2 & 8.39 & n.a. & 422.040 & 90.836 & 88.69 & n.a. & $\mathrm{BMB}^{\star}$ \\
\hline Total: & & & 480.524 & 102.425 & 100.00 & 0.000 & \\
\hline
\end{tabular}

Figure S66: HPLC chromatographs of $\mathbf{3 b k}$ 


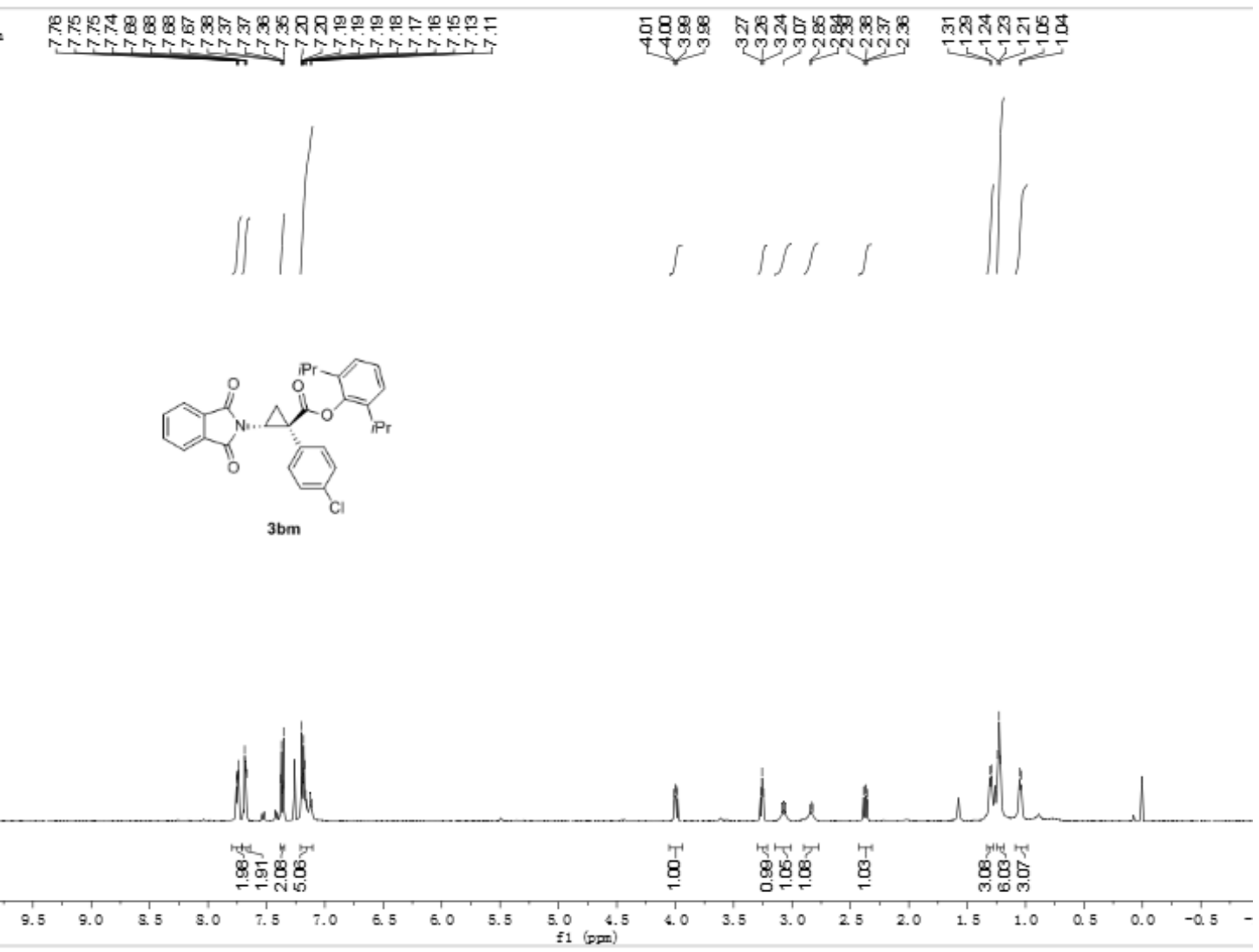

Figure S67: ${ }^{1} \mathrm{H}$ NMR $\left(500 \mathrm{MHz}, \mathrm{CDCl}_{3}\right)$ spectrum for $\mathbf{3 b m}$

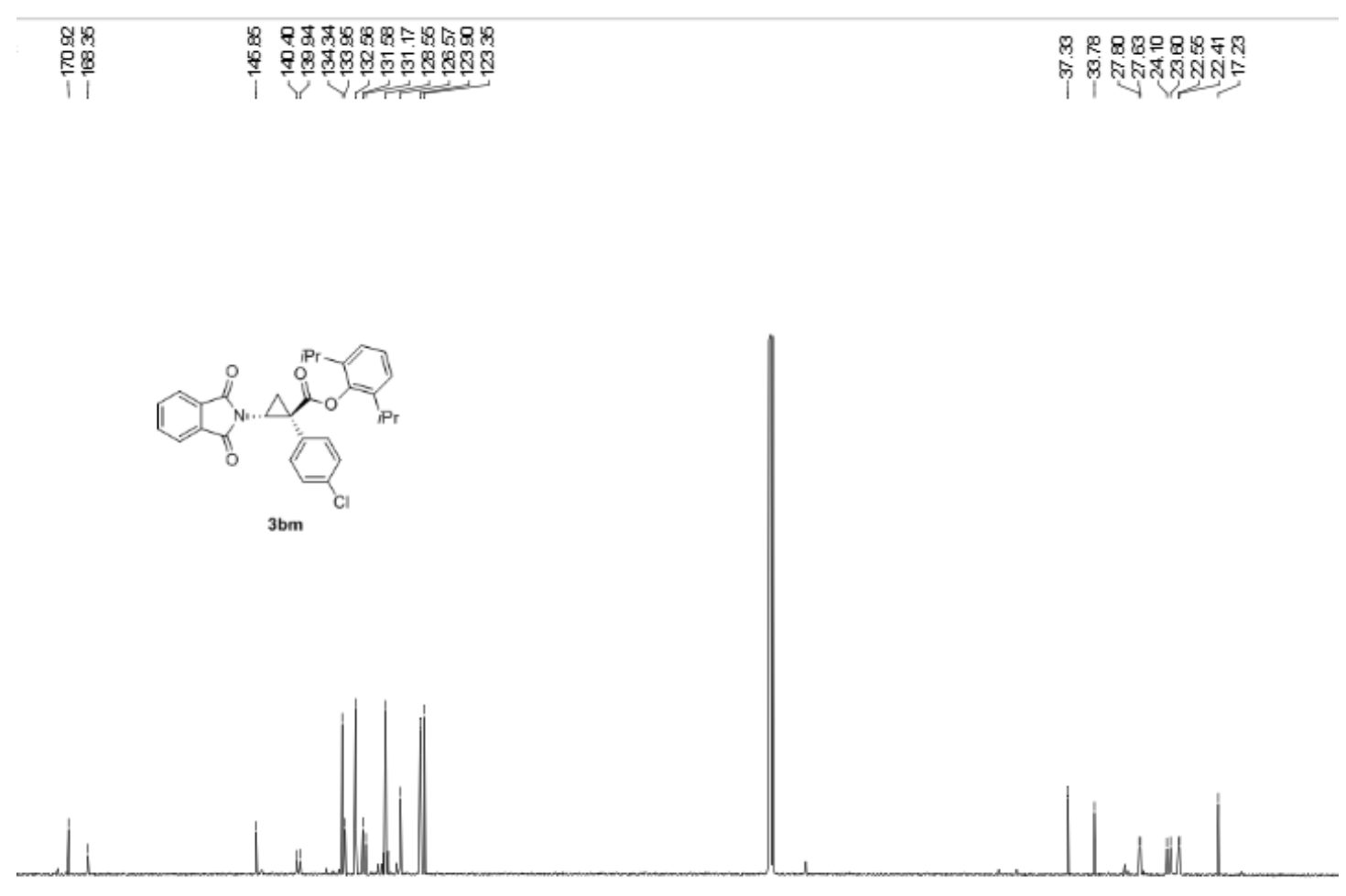

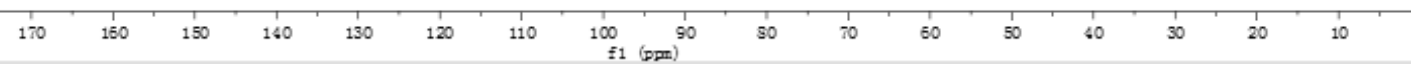

Figure S68: ${ }^{13} \mathrm{C}$ NMR $\left(125 \mathrm{MHz}, \mathrm{CDCl}_{3}\right)$ spectrum for $\mathbf{3 b m}$ 


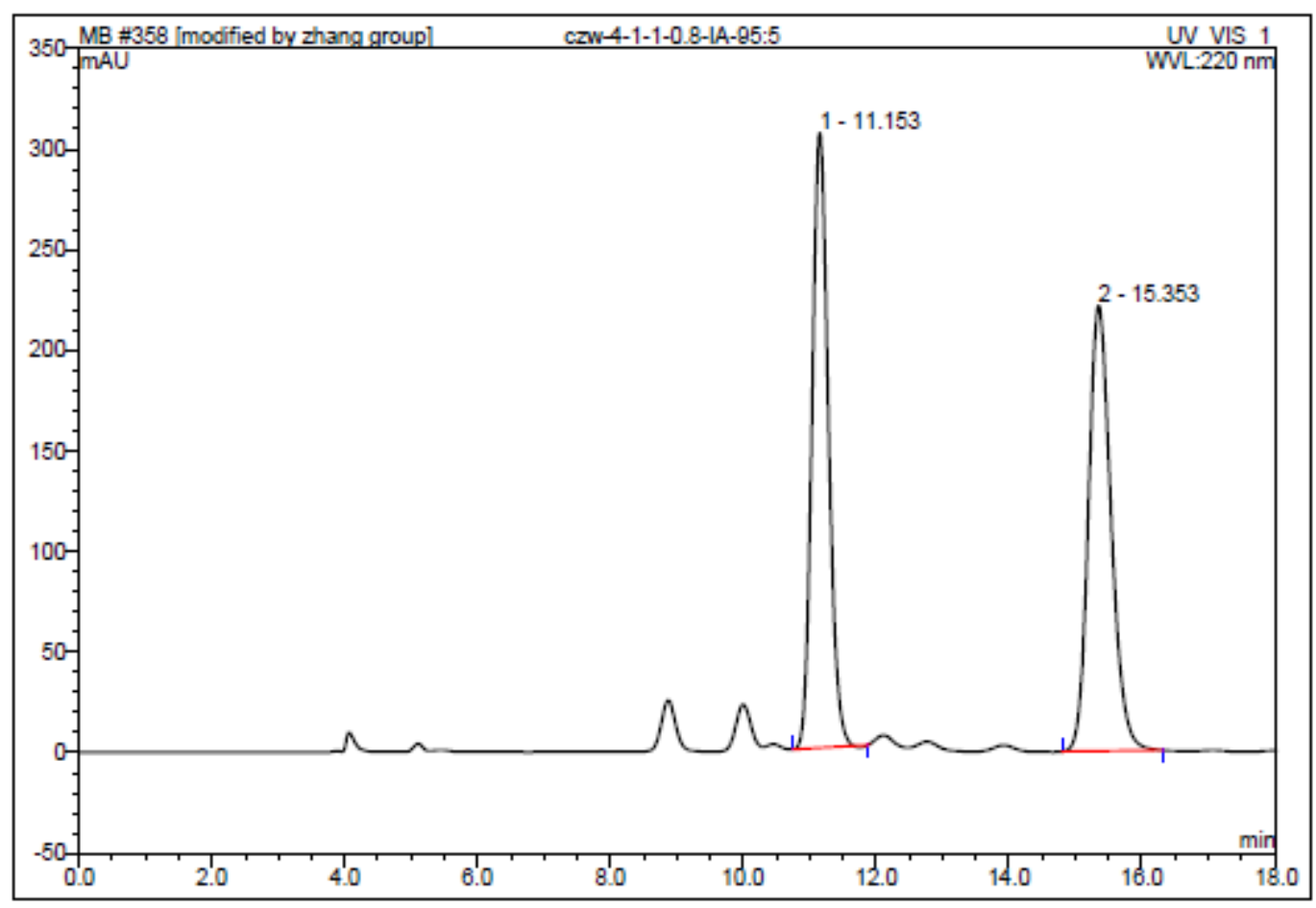

\begin{tabular}{|r|ccrrrrr|}
\hline No. & $\begin{array}{c}\text { Ret.Time } \\
\text { min }\end{array}$ & Peak Name & $\begin{array}{c}\text { Height } \\
\text { mAU }\end{array}$ & $\begin{array}{c}\text { Area } \\
\text { mAU*min }\end{array}$ & $\begin{array}{c}\text { Rel.Area } \\
\%\end{array}$ & Amount & Type \\
\hline 1 & 11.15 & n.a. & 305.712 & 87.046 & 49.96 & n.a. & BMB $^{\star}$ \\
2 & 15.35 & n.a. & 221.468 & 87.197 & 50.04 & n.a. & BMB $^{\star}$ \\
\hline Total: & & & 527.181 & 174.243 & 100.00 & 0.000 & \\
\hline
\end{tabular}

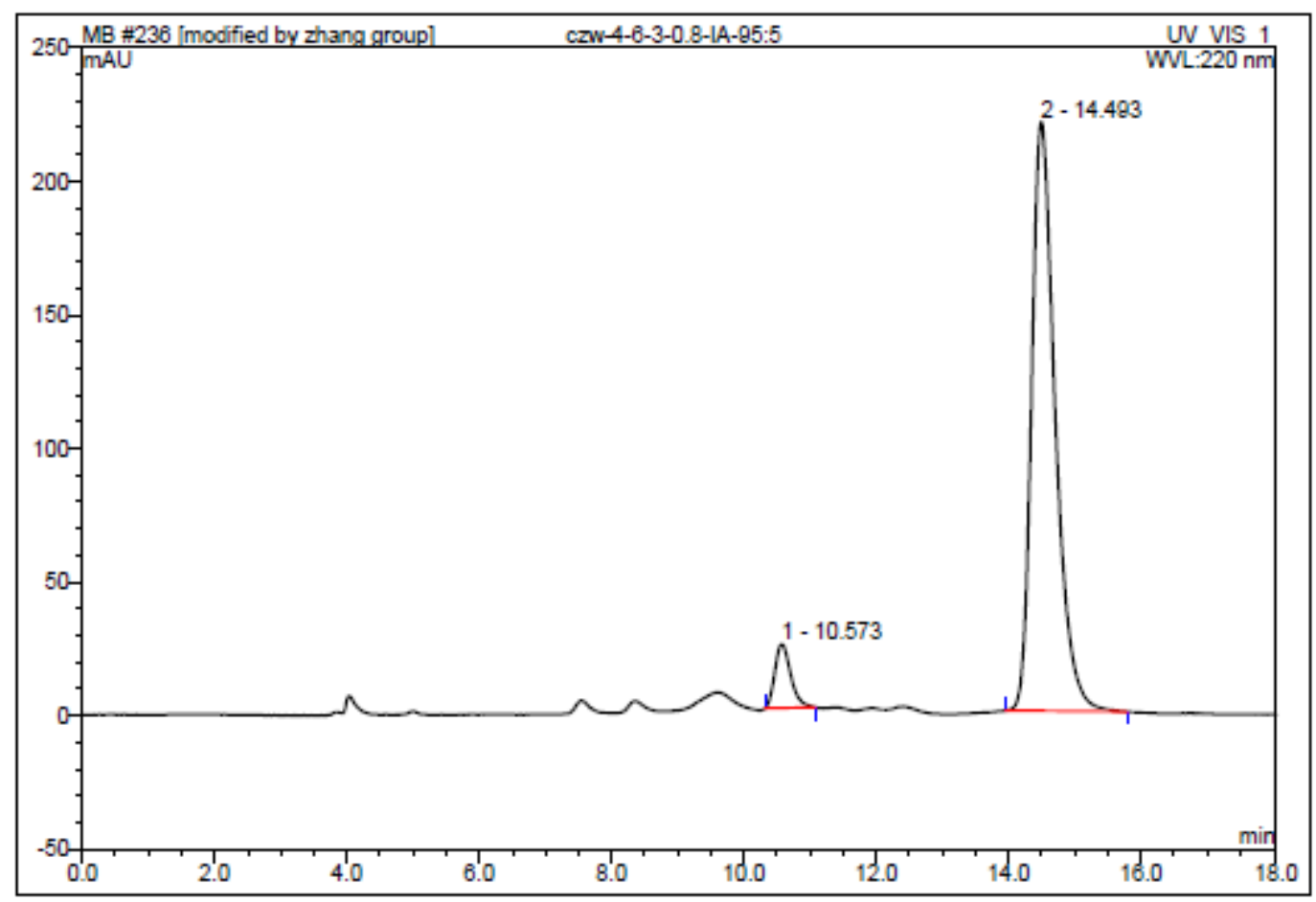

\begin{tabular}{|r|rrrrrrr|}
\hline No. & $\begin{array}{c}\text { Ret.Time } \\
\text { min }\end{array}$ & Peak Name & $\begin{array}{c}\text { Height } \\
\text { mAU }\end{array}$ & $\begin{array}{c}\text { Area } \\
\text { mAU*min }\end{array}$ & $\begin{array}{r}\text { Rel.Area } \\
\%\end{array}$ & Amount & Type \\
\hline 1 & 10.57 & n.a. & 23.958 & 6.679 & 6.86 & n.a. & BMB $^{\star}$ \\
2 & 14.49 & n.a. & 220.439 & 90.691 & 93.14 & n.a. & BMB $^{\star}$ \\
\hline Total: & & & 244.398 & 97.369 & 100.00 & 0.000 & \\
\hline
\end{tabular}


Figure S69: HPLC chromatographs of $\mathbf{3 b m}$

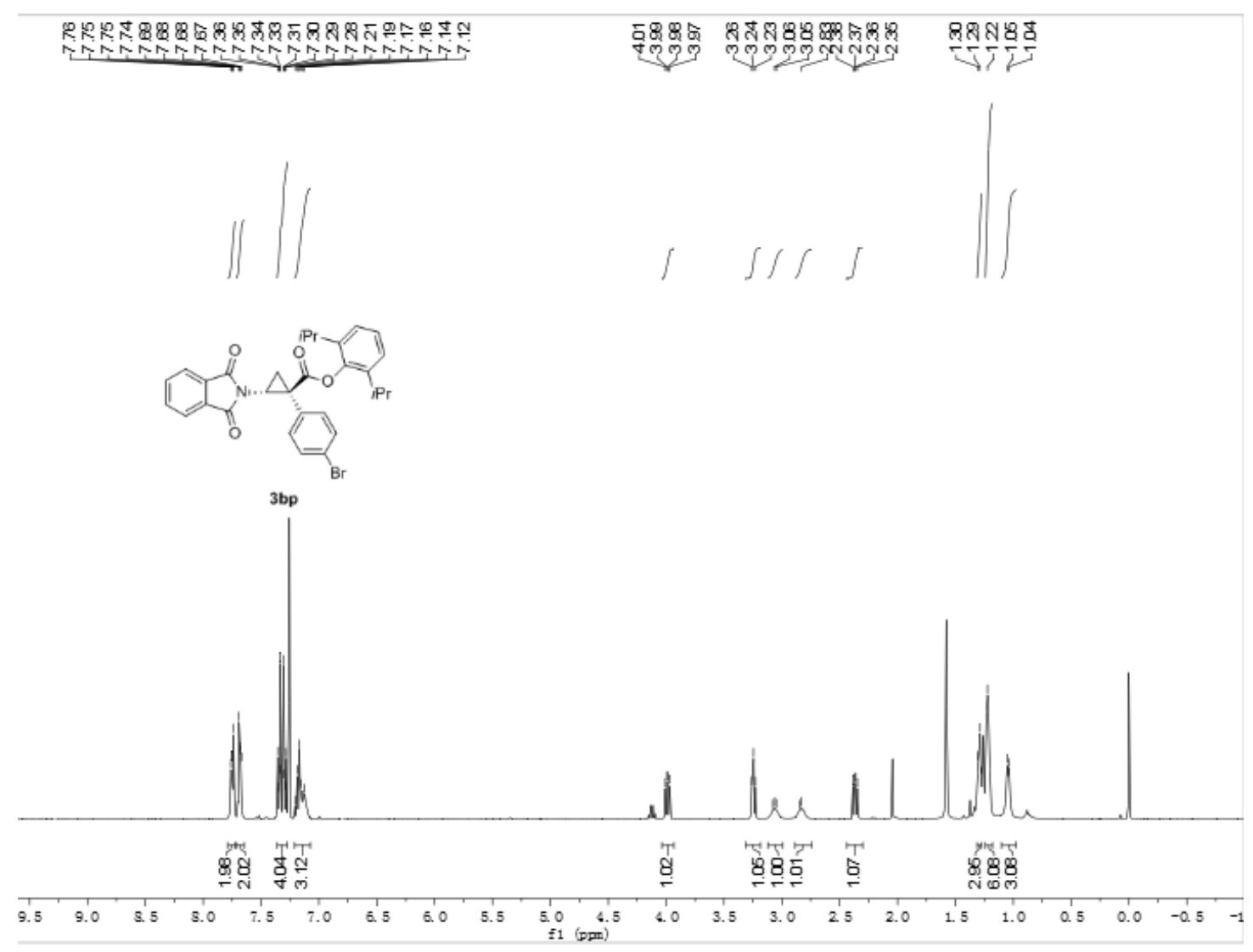

Figure S70: ${ }^{1} \mathrm{H} \mathrm{NMR}\left(400 \mathrm{MHz}, \mathrm{CDCl}_{3}\right)$ spectrum for $\mathbf{3 b p}$ 


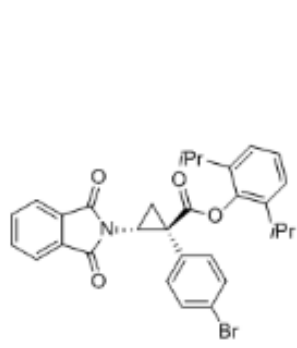

$3 \mathrm{bp}$

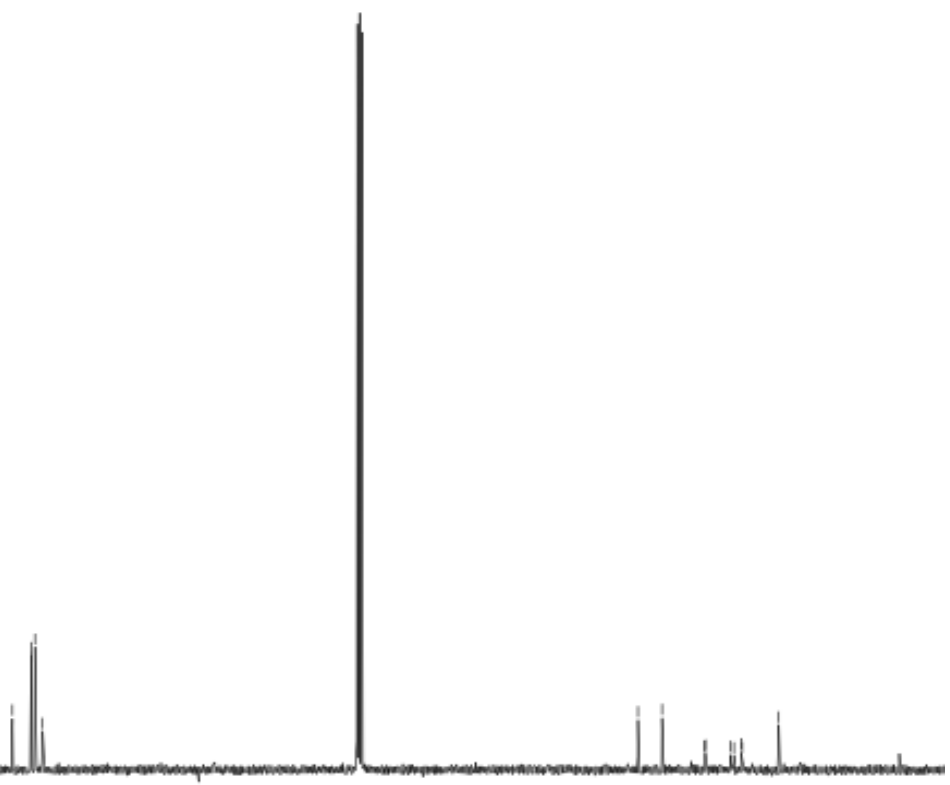

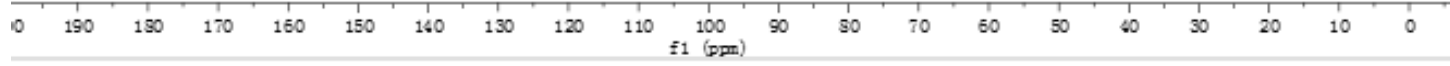

Figure S71: ${ }^{13} \mathrm{C}$ NMR $\left(100 \mathrm{MHz}, \mathrm{CDCl}_{3}\right)$ spectrum for $\mathbf{3 b p}$

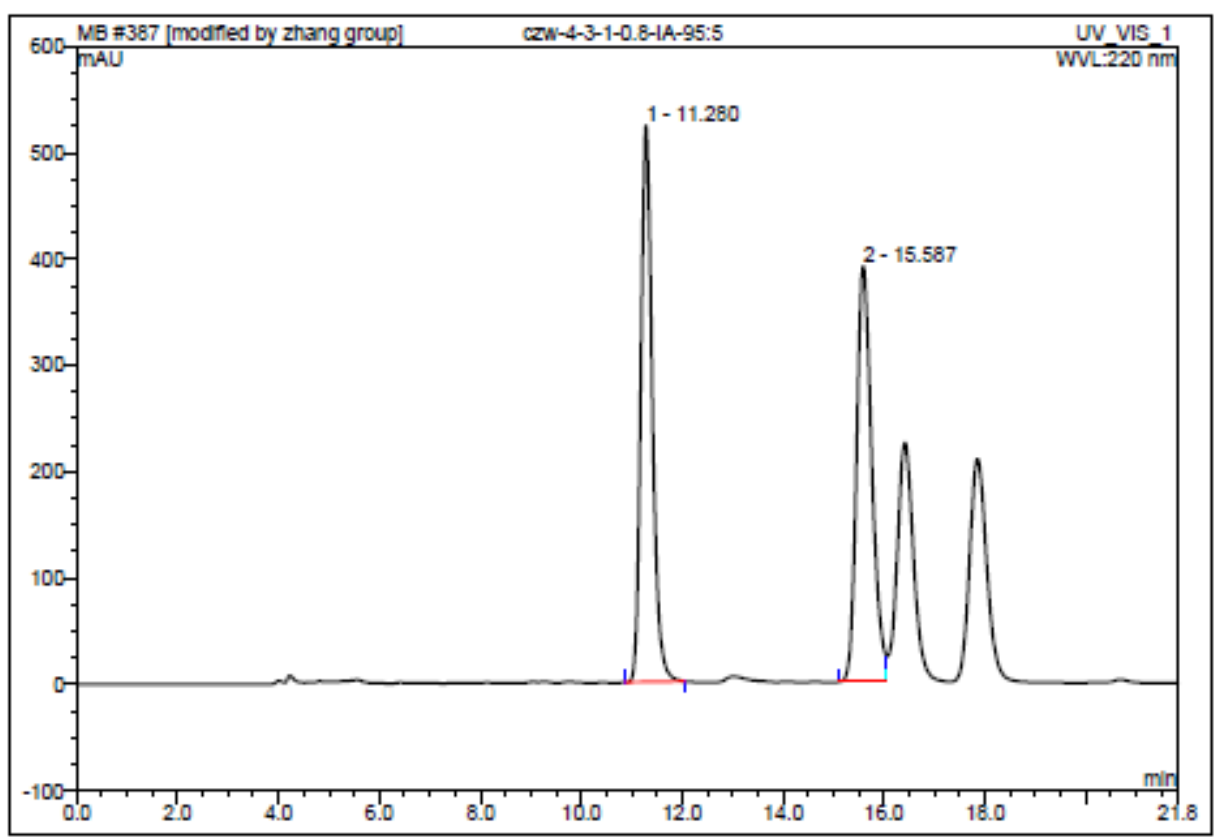

\begin{tabular}{|r|ccccccc|}
\hline No. & $\begin{array}{c}\text { Ret.Time } \\
\text { min }\end{array}$ & Peak Name & $\begin{array}{c}\text { Height } \\
\text { mAU }\end{array}$ & $\begin{array}{c}\text { Area } \\
\text { mAU*min }\end{array}$ & $\begin{array}{c}\text { Rel.Area } \\
\text { \% }\end{array}$ & Amount & Type \\
\hline 1 & 11.28 & n.a. & 522.858 & 138.536 & 50.21 & n.a. & BMB $^{*}$ \\
2 & 15.59 & n.a. & 390.665 & 137.351 & 49.79 & n.a. & BM $^{*}$ \\
\hline Total: & & & 913.523 & 275.886 & 100.00 & 0.000 & \\
\hline
\end{tabular}




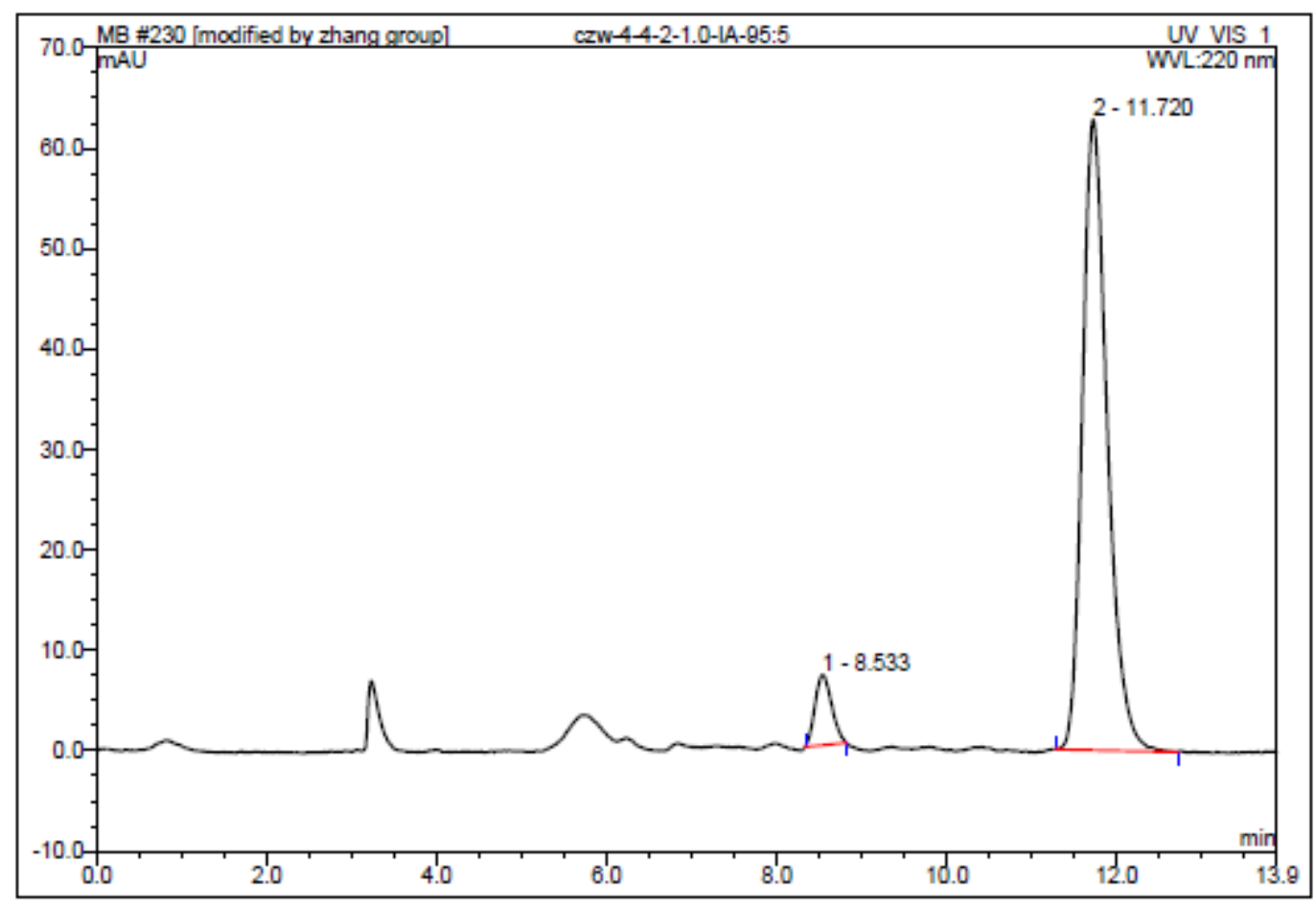

\begin{tabular}{|r|rrrrrrr|}
\hline No. & $\begin{array}{c}\text { Ret.Time } \\
\text { min }\end{array}$ & Peak Name & $\begin{array}{c}\text { Height } \\
\text { mAU }\end{array}$ & $\begin{array}{c}\text { Area } \\
\text { mAUmin }\end{array}$ & $\begin{array}{r}\text { Rel.Area } \\
\%\end{array}$ & Amount & Type \\
\hline 1 & 8.53 & n.a. & 7.011 & 1.588 & 6.92 & n.a. & BMB $^{\star}$ \\
2 & 11.72 & n.a. & 62.771 & 21.359 & 93.08 & n.a. & BMB $^{\star}$ \\
\hline Total: & & & 69.782 & 22.948 & 100.00 & 0.000 & \\
\hline
\end{tabular}

Figure S72: HPLC chromatographs of 3bp 


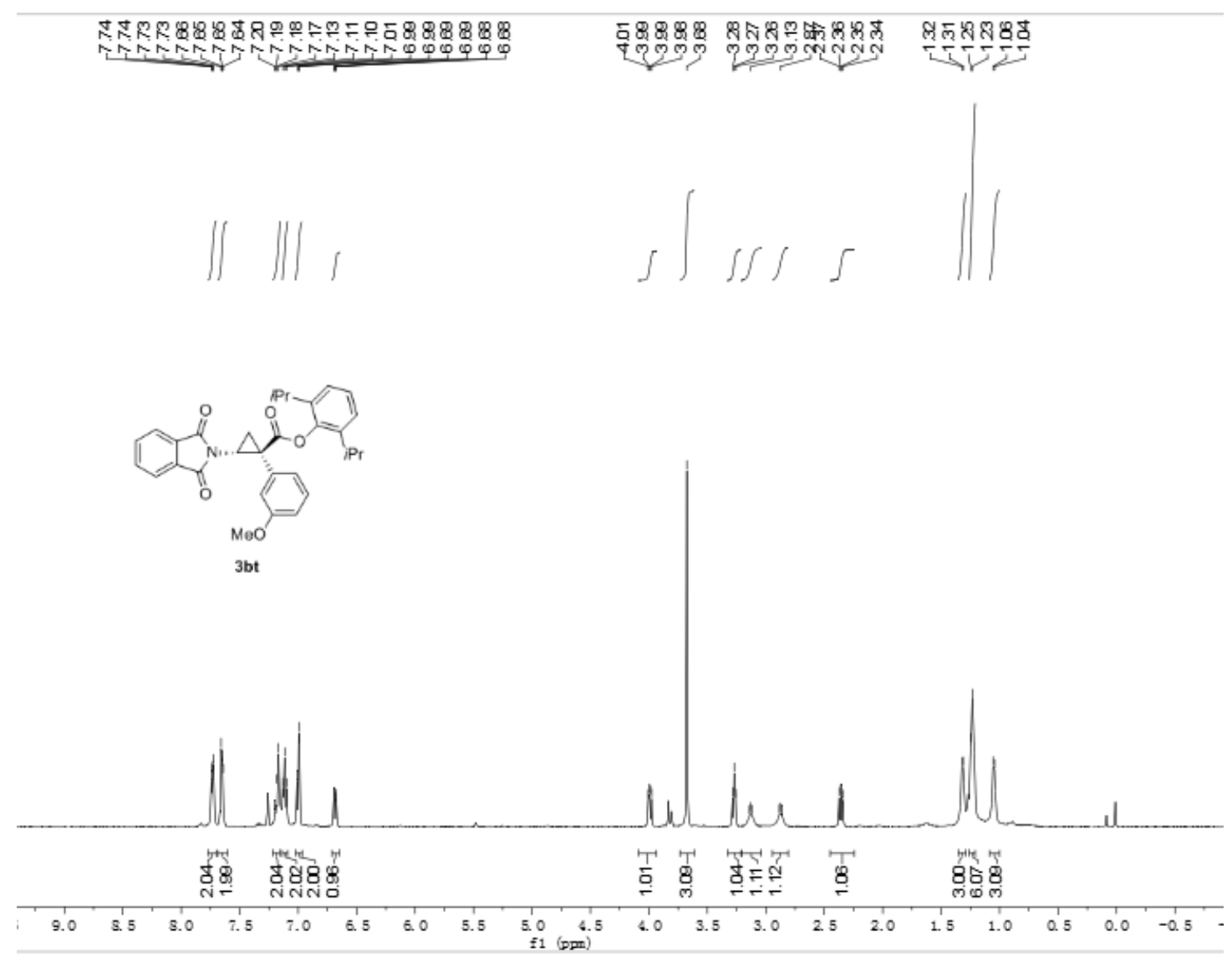

Figure S73: ${ }^{1} \mathrm{H}$ NMR $\left(500 \mathrm{MHz}, \mathrm{CDCl}_{3}\right)$ spectrum for $\mathbf{3 b t}$

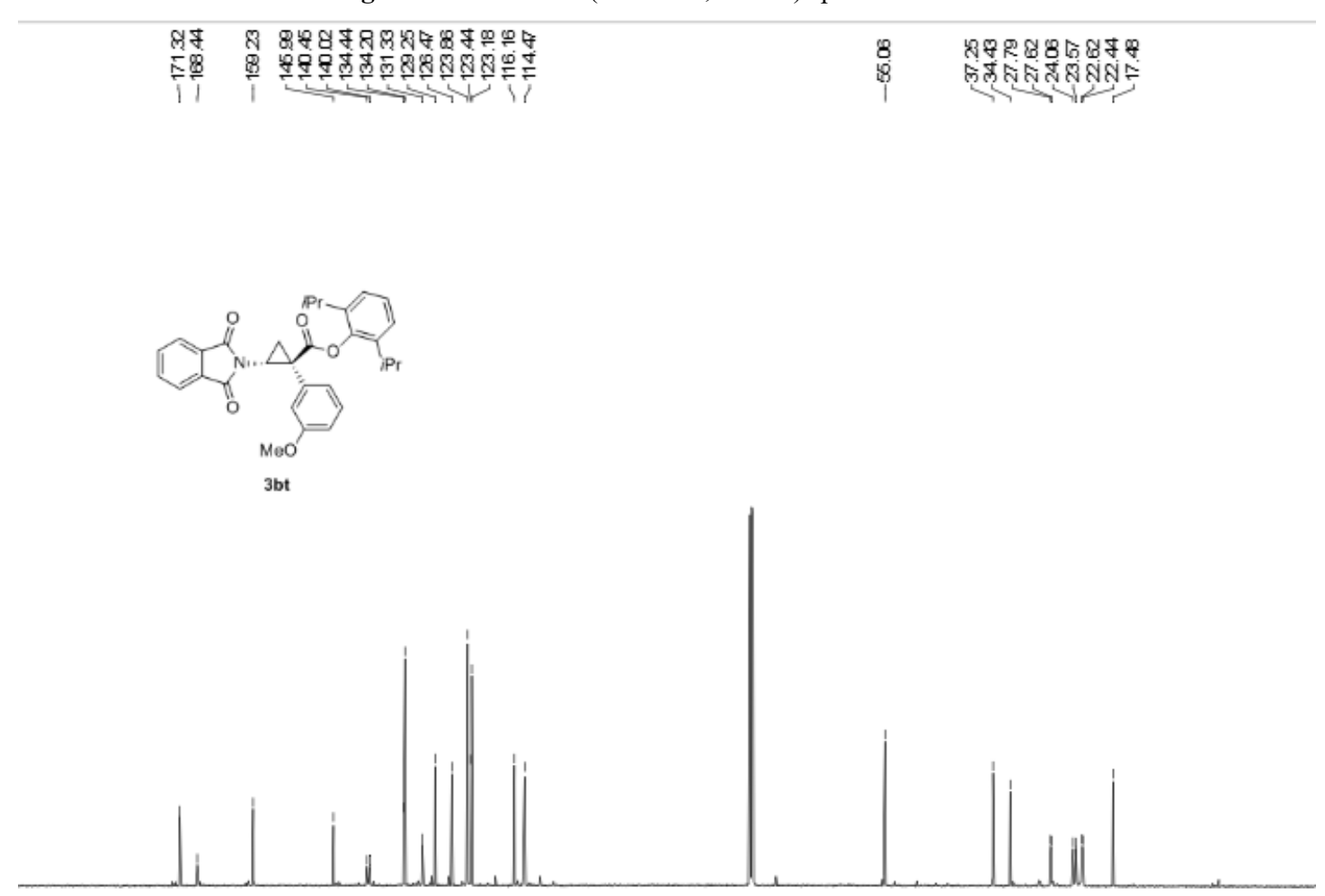

$\begin{array}{llllllllllllllllllllll}190 & 190 & 170 & 160 & 150 & 160 & 130 & 120 & 110 & 100 & 90 & 50 & 70 & 60 & 50 & 40 & 30 & 20 & 10 & 0 & -10\end{array}$ 
Figure S74: ${ }^{13} \mathrm{C}$ NMR (125 MHz, $\left.\mathrm{CDCl}_{3}\right)$ spectrum for $\mathbf{3 b t}$

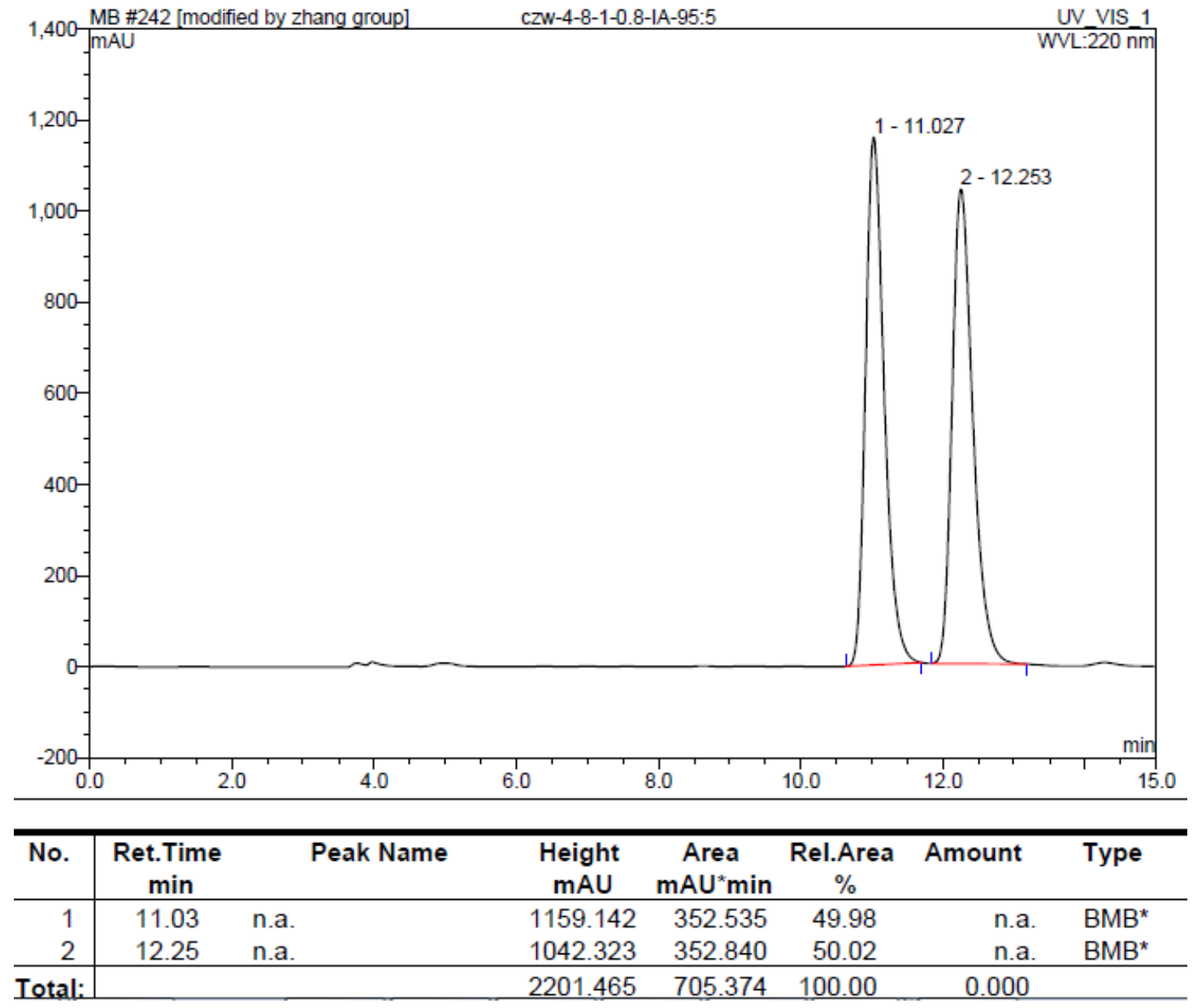




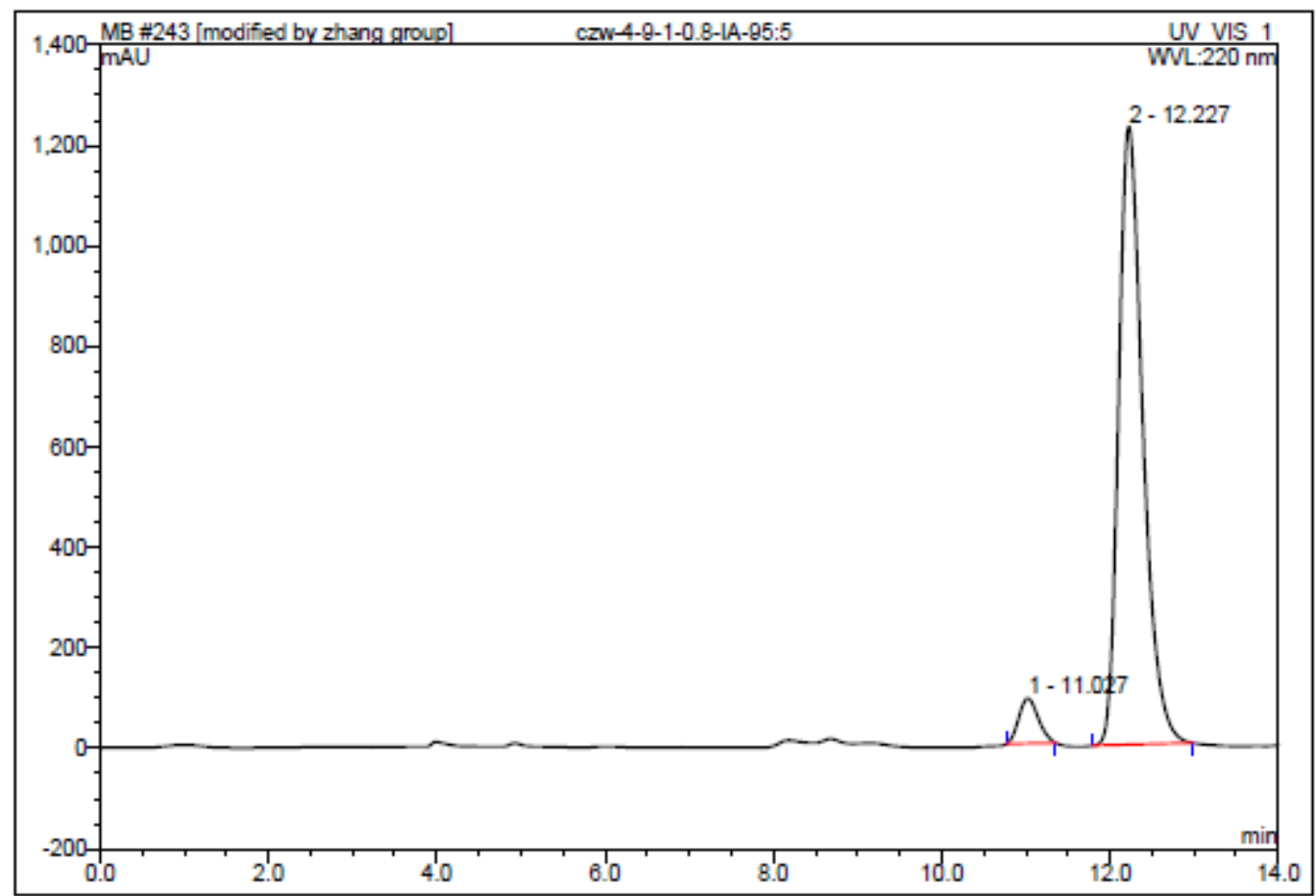

\begin{tabular}{|r|ccrrrrr|}
\hline No. & $\begin{array}{c}\text { Ret.Time } \\
\text { min }\end{array}$ & Peak Name & $\begin{array}{c}\text { Height } \\
\text { mAU }\end{array}$ & $\begin{array}{c}\text { Area } \\
\text { mAU*min }\end{array}$ & $\begin{array}{r}\text { Rel.Area } \\
\%\end{array}$ & Amount & Type \\
\hline 1 & 11.03 & n.a. & 89.471 & 24.020 & 5.51 & n.a. & BMB $^{\star}$ \\
2 & 12.23 & n.a. & 1229.754 & 411.829 & 94.49 & n.a. & BMB $^{\star}$ \\
\hline Total: & & & 1319.225 & 435.849 & 100.00 & 0.000 & \\
\hline
\end{tabular}

Figure S75: HPLC chromatographs of $3 \mathbf{b t}$

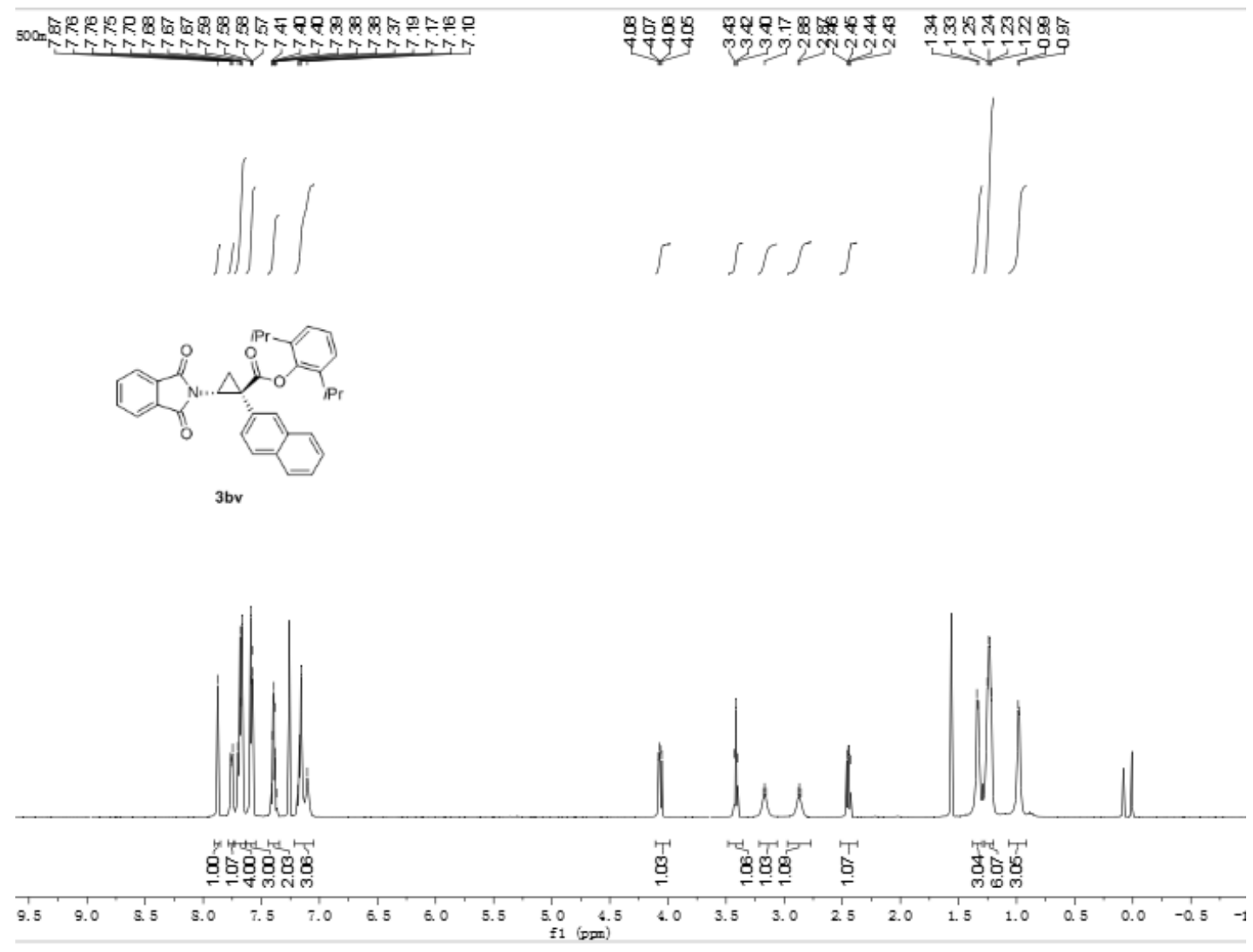


Figure S76: ${ }^{1} \mathrm{H} \mathrm{NMR}\left(500 \mathrm{MHz}, \mathrm{CDCl}_{3}\right)$ spectrum for $\mathbf{3 b v}$

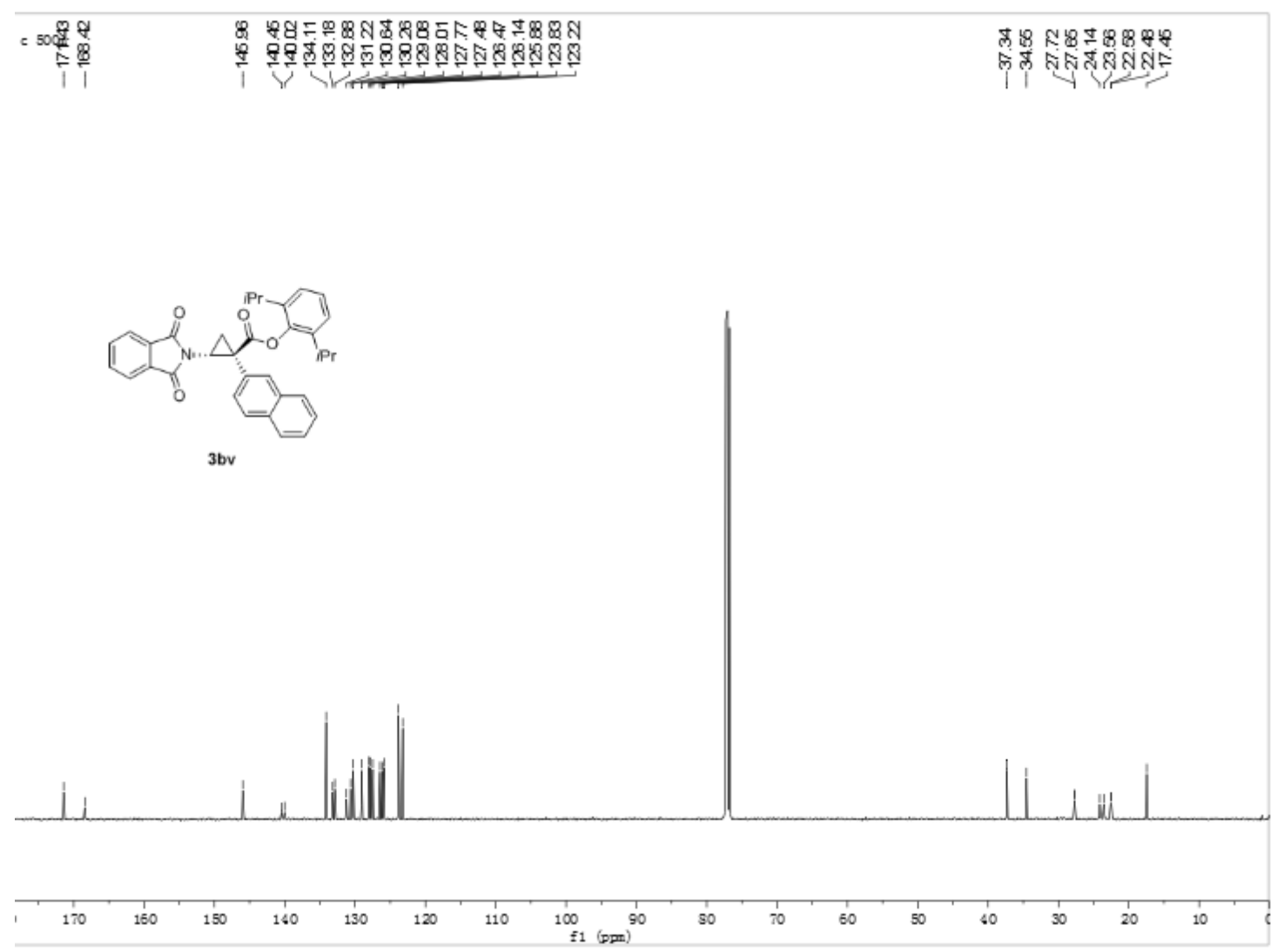

Figure S77: ${ }^{13} \mathrm{C}$ NMR $\left(125 \mathrm{MHz}, \mathrm{CDCl}_{3}\right)$ spectrum for $\mathbf{3 b v}$ 


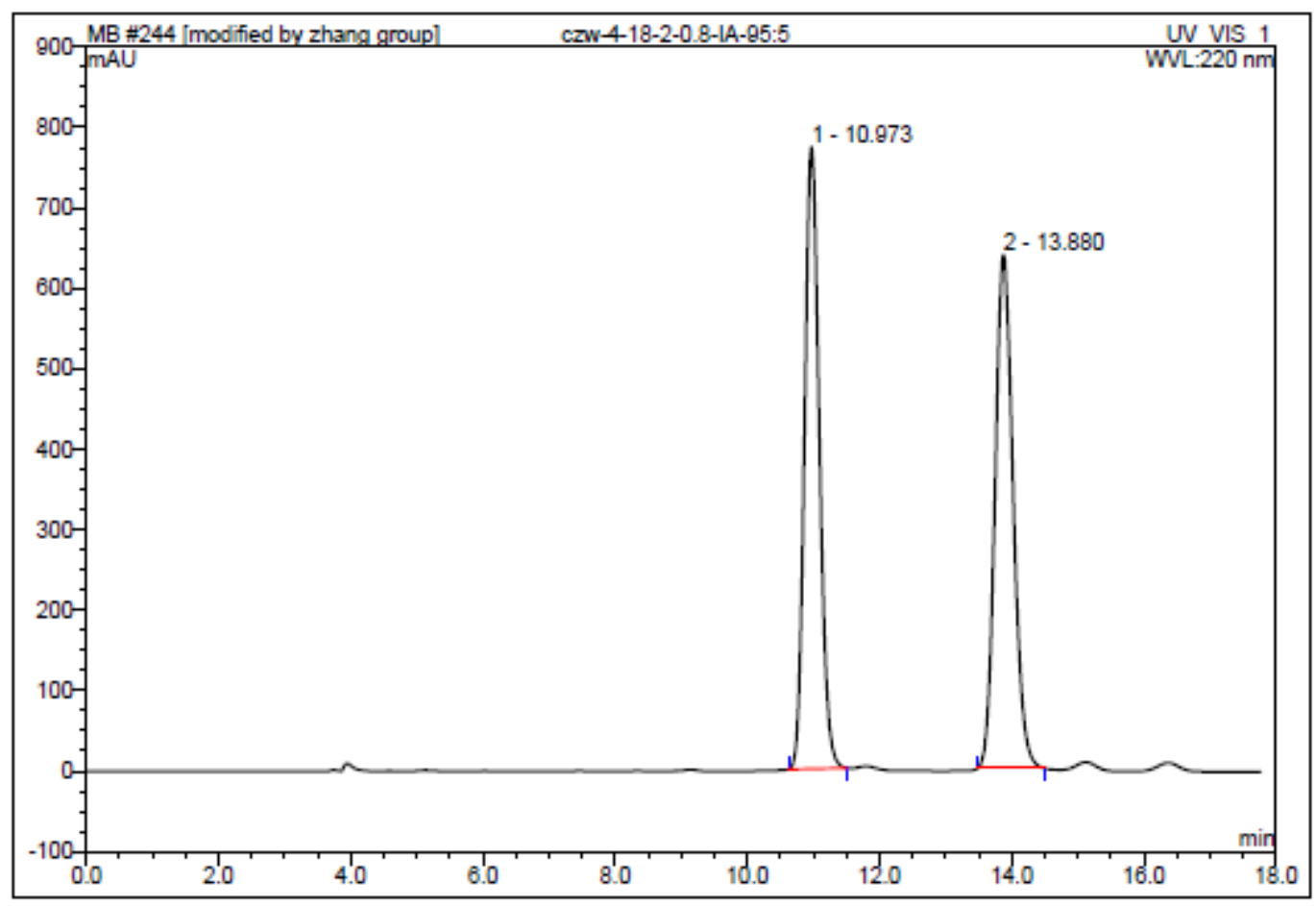

\begin{tabular}{|r|ccrrrrr|}
\hline No. & $\begin{array}{c}\text { Ret.Time } \\
\text { min }\end{array}$ & Peak Name & $\begin{array}{c}\text { Height } \\
\text { mAU }\end{array}$ & $\begin{array}{c}\text { Area } \\
\text { mAU*min }\end{array}$ & $\begin{array}{c}\text { Rel.Area } \\
\%\end{array}$ & Amount & Type \\
\hline 1 & 10.97 & n.a. & 773.046 & 199.861 & 49.71 & n.a. & BMB $^{\star}$ \\
2 & 13.88 & n.a. & 638.042 & 202.172 & 50.29 & n.a. & BMB $^{\star}$ \\
\hline Total: & & & 1411.088 & 402.033 & 100.00 & 0.000 & \\
\hline
\end{tabular}

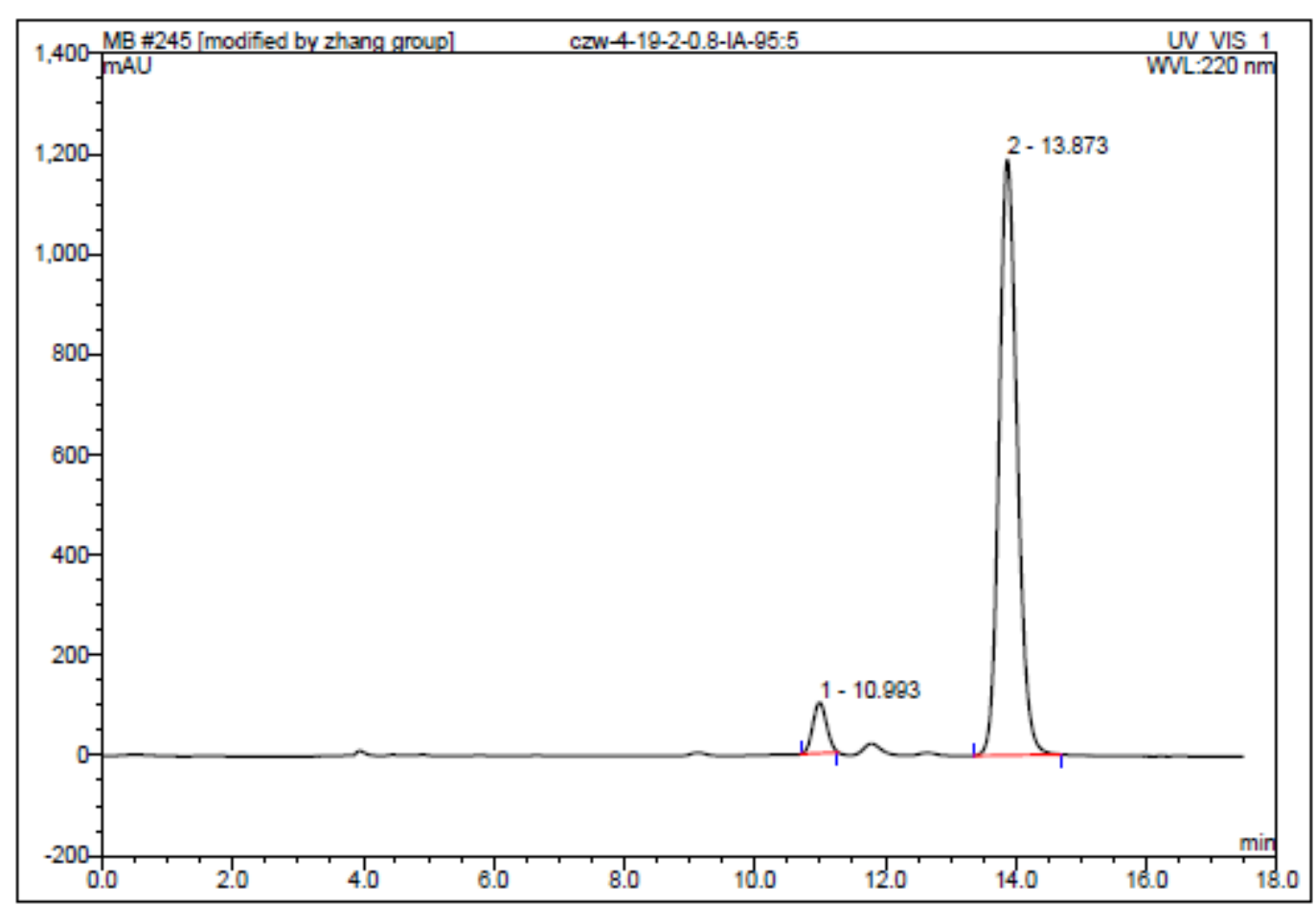

\begin{tabular}{|r|ccrrrrr|}
\hline No. & $\begin{array}{c}\text { Ret.Time } \\
\text { min }\end{array}$ & Peak Name & $\begin{array}{c}\text { Height } \\
\text { mAU }\end{array}$ & $\begin{array}{c}\text { Area } \\
\text { mAU*min }\end{array}$ & $\begin{array}{r}\text { Rel.Area } \\
\%\end{array}$ & Amount & Type \\
\hline 1 & 10.99 & n.a. & 101.123 & 24.606 & 6.06 & n.a. & BMB $^{\star}$ \\
2 & 13.87 & n.a. & 1187.382 & 381.336 & 93.94 & n.a. & BMB $^{\star}$ \\
\hline Total: & & & 1288.505 & 405.942 & 100.00 & 0.000 & \\
\hline
\end{tabular}


Figure S78: HPLC chromatographs of 3bv 